CIRJE-F-1085

\title{
Social Image Concern and Reference Point Formation
}

Yosuke Hashidate

Waseda University and Japan Society for the Promotion of Science

May 2018; Revised in January 2020

CIRJE Discussion Papers can be downloaded without charge from:

http://www.cirje.e.u-tokyo.ac.jp/research/03research02dp.html

Discussion Papers are a series of manuscripts in their draft form. They are not intended for circulation or distribution except as indicated by the author. For that reason Discussion Papers may not be reproduced or distributed without the written consent of the author. 


\title{
Social Image Concern and Reference Point Formation*
}

\author{
YOSUKE HashidATE ${ }^{\dagger}$
}

First Draft: August 8, 2017; This Draft: December 17, 2019

\begin{abstract}
This paper provides an axiomatic foundation for social image concerns, in the presence of context-dependence. This paper allows for context-dependence based on choice sets, and studies how context-dependence impacts social image concerns. In particular, this paper investigates a decision maker who exhibits various social emotions stemming from his own intrinsic reference point, which may not be captured by conforming to social norm. By uniquely identifying the building blocks of the model, this paper studies a relationship between social image and choice-set effects in terms of pride-seeking preferences, shameaverse preferences, and temptation-driven preferences. Moreover, a relationship between image-conscious utility and outcome-based utility such as inequity aversion is discussed. A relationship between image-conscious utility and intention-based utility such as intrinsic reciprocity is also discussed.
\end{abstract}

Keywords: Social Image; Social Emotions; Reference Point Formation; Context-Dependence; Image-Conscious Utilitarian.

JEL Classification Numbers: D01, D63, D64, D91.

${ }^{*}$ The earlier version of this paper is presented by "Reference-Dependent Fairness Attitudes: An Axiomatic Approach" and "A Cognitive Foundation for Social Image Concerns." I am indebted to my adviser Akihiko Matsui for his unique guidance, constant support, and encouragement, when I was a Ph.D. student at the University of Tokyo. I am very grateful to Yoichiro Fujii, Youichiro Higashi, Jay Lu, Kota Saito, and Norio Takeoka, and anonymous referees for their invaluable suggestions and comments. I would like to thank the participants at UT Summer School in Economics 2017 (University of Tokyo), EEA-ESEM 2017 (ISCTE-IUL Campus, Lisbon), JEA 2017 Autumn Meeting (Aoyama Gakuin University, Tokyo), and Economics Research Meeting (Osaka Sangyo University). The part of this research was done when I was a research associate at CIRJE, the Graduate School of Economics, the University of Tokyo. I am thankful for their hospitality. Of course, all remaining errors are mine.

${ }^{\dagger}$ Waseda Business School, Waseda University and Japan Society for the Promotion of Science; Email:

yosukehashidate@gmail.com 


\section{Contents}

1 Introduction 1

2 Axioms 2

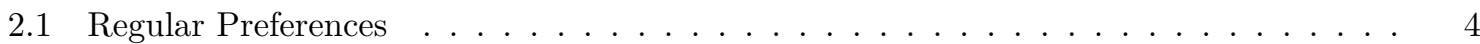

2.2 Image Concern and Reference-Point Formation . . . . . . . . . . . . . . . . . . . . 6

2.3 Relaxing Independence . . . . . . . . . . . . . . . . . . . . . . . 7

3 Result

3.1 Representation Theorem ............................8 8

3.2 Uniqueness Result ... . . . . . . . . . . . . . . . . . . . . . 13

4 The Effects on Social Emotions 13

4.1 Altruism, Selfishness and Inequity Aversion . . . . . . . . . . . . . . . . . . . . 13

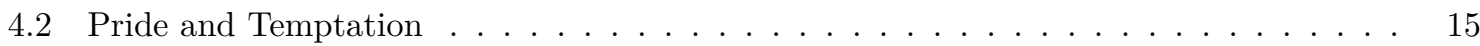

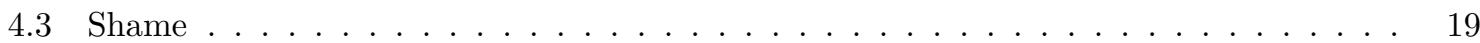

5 Reference Point Formation $\quad \mathbf{2 0}$

5.1 Comparative Statics on Reference Points . . . . . . . . . . . . . . . . . . . . . . . 21

5.2 Choice Set Effects . . . . . . . . . . . . . . . . . . . . . . 21

6 A Relationship between Social Image and Reciprocity 23

6.1 Nash Equilibrium . . . . . . . . . . . . . . . . . . . . . . . . . . 24

6.2 Intrinsic Reciprocity . . . . . . . . . . . . . . . . . . . . . . 26

$\begin{array}{lll}7 & \text { Literature Review } & 28\end{array}$

8 Concluding Remarks $\quad 32$

$\begin{array}{lll}\text { A Proof of Theorem } 1 & 34\end{array}$

A.1 Sufficiency Part . . . . . . . . . . . . . . . . . . . . . . . . . . . 34

A.2 Necessity Part. . . . . . . . . . . . . . . . . . . . . . . . 43

B Proof of Propositions $\quad 44$

B.1 Proof of Proposition 1 . . . . . . . . . . . . . . . . . . . . . . . 44

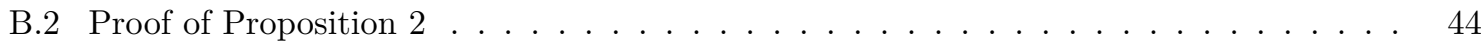

B.3 Proof of Proposition 3 . . . . . . . . . . . . . . . . . . . . . . . . . . . 45

B.4 Proof of Proposition 4 . . . . . . . . . . . . . . . . . . . . . . . . 45

B.5 Proof of Proposition $5 \ldots \ldots \ldots \ldots$

B.6 Proof of Proposition $6 \ldots \ldots \ldots \ldots$

B.7 Proof of Proposition $7 \ldots \ldots \ldots \ldots$. . . . . . . . . . . . . . . . . 47

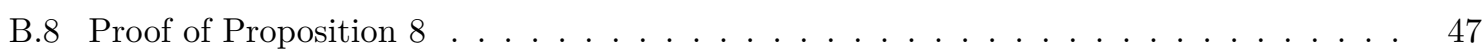

B.9 Proof of Proposition 9 . . . . . . . . . . . . . . . . . . . . . . . . . 48

B.10 Proof of Proposition 10 . . . . . . . . . . . . . . . . . . . . . . . . . 48

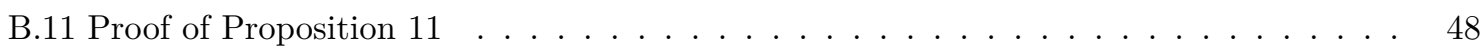




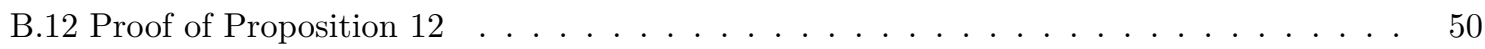

$\begin{array}{ll}\text { C Proofs of Corollaries } & 51\end{array}$

C.1 Proof of Corollary $1 \ldots \ldots \ldots \ldots \ldots \ldots \ldots$

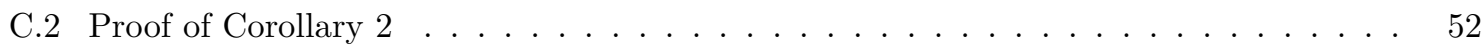

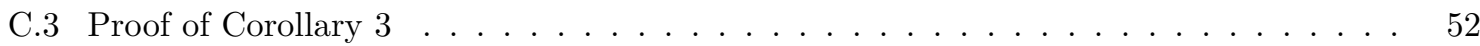

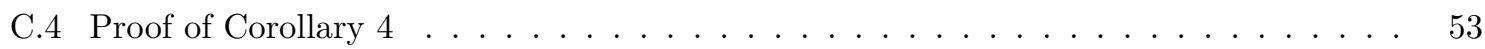

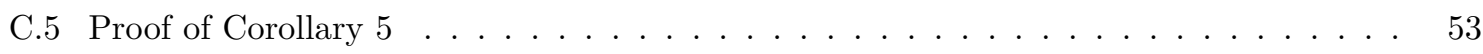

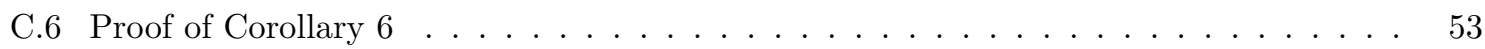

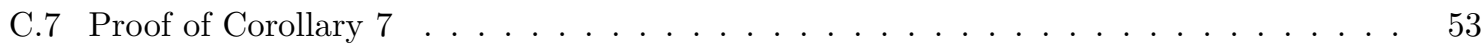

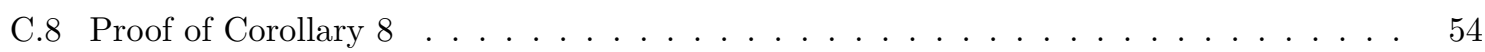

D Examples $\quad \mathbf{5 4}$

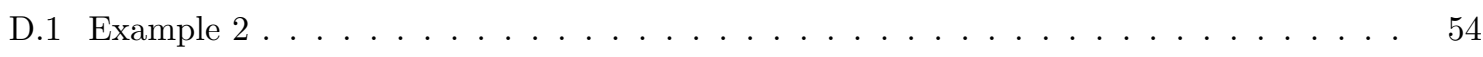

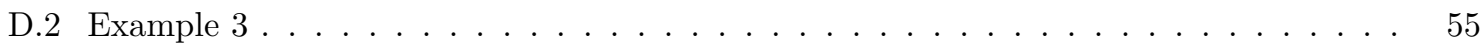




\section{Introduction}

This paper develops an axiomatic context-dependent model of social image concerns. By studying preferences over menus of allocations, i.e., choice sets, this paper axiomatizes a reference-dependent image-conscious utilitarian (henceforth, RDIC), and presents a unified model of three existing studies on social image concerns: (i) Dillenberger and Sadowski (2012), (ii) Evren and Minardi (2017), and (iii) Saito (2015).

In social image concerns, it is postulated that the decision maker cares about howhis choice behavior is perceived by other passive agents. ${ }^{1}$ The preferences-over-menus framework has axiomatically identified social emotions like shame of acting selfishly, pride of acting altruistically, and temptation to act selfishly. ${ }^{2}$

The levels of social emotions depend on contexts. ${ }^{3}$ This paper focuses only on a contextdependence stemming from choice opportunities. Social emotions can generally change through choice sets. Since emotions are not stable, the resulting behavior can be referencedependent. For instance, if a choice set includes a selfish option, the decision maker feels temptation to act selfishly, even though he tries to take prosocial behavior.

\section{Motivation}

Dictator Games. Take, for instance, a menu $\{(1,0),(0,1)\} \cdot{ }^{4}$ Consider the simplified dictator game in which the dictator chooses the allocation from the menu. Suppose that there is a social norm, and that there is no cost on conforming to the norm. In this sense, any social emotions do not exhibit.

There are two considerable scenarios. One scenario is that the dictator may not feel shame if he chooses $(1,0)$ because the social norm may deem it acceptable to be selfish. It is too costly to act altruistically. Another scenario is that the dictator may not feel pride if he chooses $(0,1)$, because the social norm may dictate selfishness at all costs. The reference point is a "feasible" allocation chosen by society, $(1,0)$ or $(0,1)$, respectively.

However, alternative possibility is that he still feels shame even if the social norm make $(1,0)$ acceptable, or that she still feels pride even if $(0,1)$ is the social norm. These social emotions can occur since simplistic moral maxims like "selfishness is bad" or "selfishness is

\footnotetext{
${ }^{1}$ See the experimental studies Dana et al. (2006), Kawamura et al. (2019), etc. Bursztyn and Jensen (2017) is an excellent survey on the study of social image concerns.

${ }^{2}$ This framework has a two-stage decision problem. At the first (ex-ante) stage, the decision maker chooses a menu; at the second (ex-post) stage, he chooses an alternative from the first-stage chosen menu. Kreps (1979) introduces this framework, and Dekel et al. (2001) generalize it. See Figure 1.

${ }^{3}$ These socially emotional aspects can generally depend on contexts like choice opportunities, the other players' personality types, the other players' past actions, etc.

${ }^{4} \mathrm{I}$ appreciate an anonymous referee for the suggestion of the insightful example. For simplicity, $(1,0)$ is the allocation that the dictator obtains the payoff 1 , and the recipient obtains the payoff 0 . In the same way, $(0,1)$ is the allocation that the dictator obtains the payoff 0 , and the recipient obtains the payoff 1 .
} 
good" are at play instinctively, regardless of the particular contexts in the presence of social norms.

Endogenous Reference Points. The reference point is a more complicated psychological construct that may have a context-dependent component, if difference choice situations exhibit different social emotions. For example, in a menu, the decision maker feels pride of acting altruistically. On the other hand, in another menu, the same decision maker feels temptation to act selfishly. Thus, social emotions are not stale.

The reference point is also a more complicated psychological construct that may allow for an "unfeasible" allocation, if the decision maker exhibits various social emotions in some contexts. The related studies sit the reference points, the most peronally normative allocation (Dillenberger and Sadowski (2012)), the most selfish allocation (Evren and Minardi (2017)), or both (Saito (2015)). These studies identify shame, warm-glow, temptation, and pride, but there are various important social emotions like spitefulness and regret. For example, the decision maker may take selfish behavior, not only to increase his own payoff, but also to try to increase the difference between his payoff and others. The latter arise from spiteful emotion.

The objective of this paper is three-fold. First, this paper develops an axiomatic model of social image concerns, which is not captured by conforming to (social) norm. Second, this paper allows for various social emotions to study image-conscious preferences with referencedependence. We study such a context-dependence as a reference-point formation. ${ }^{5}$ Third, this paper proposes a unified model of the existing studies mentioned above.

\section{Outline}

The rest of this paper is organized as follows. In Section2, we provide the axioms in the main result of this paper. In Section 3, we state the main result of this paper (Theorem 1 and Proposition 1). In Section 4, we study social emotions. In Section 5, we study the role of reference point to impact social image concerns. In Section 6, we study a relationship between social image concerns and intrinsic reciprocity. In Section 7, we provide a literature review. Finally, Section 8 concludes. All proofs are in the Appendix.

\section{Axioms}

A Two-Stage Decision Problem. We take the framework of preferences over menus of allocations, based on previous related studies. The framework has a two-stage decision problem structure (see Figure 1).

\footnotetext{
${ }^{5}$ The reference-point formation of this paper is different from that of Köszegi and Rabin (2006).
} 


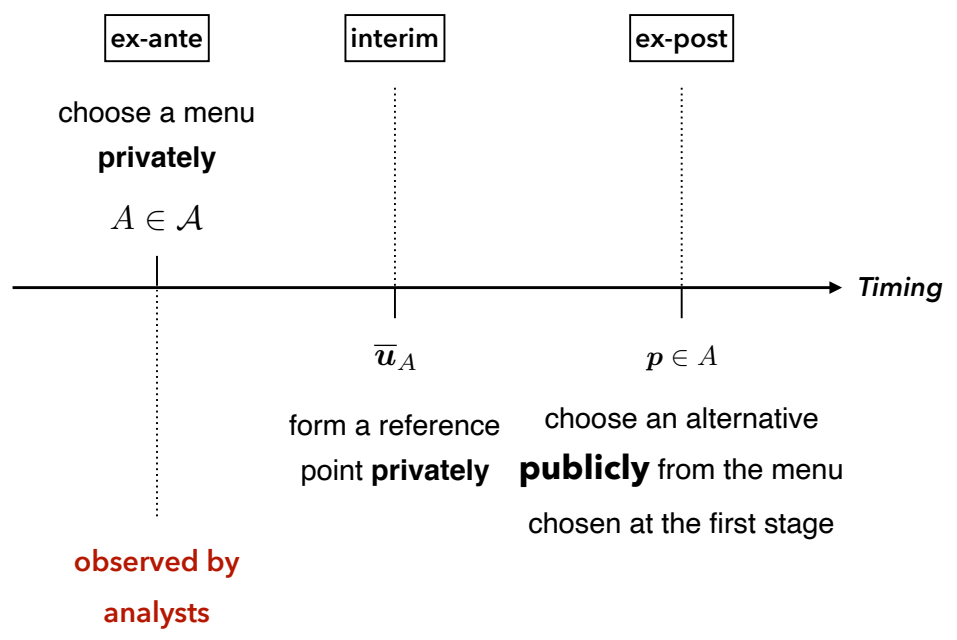

Figure 1: Two-Stage Decision Problem

Consider a dictator game. The dictator is the decision maker. At the ex-ante stage, the decision maker chooses a menu (choice set); at the ex-post stage, he chooses an allocation from his first-stage choice. The key procedure is that the choice at the ex-ante stage is not observed by the recipient; that is, recipients do not know about the set of menus (denoted by $\mathcal{A}$ in Figure 1). ${ }^{6}$ Hence, the ex-ante choices are private. On the other hand, the ex-post choices are observed publicly. The recipient can observe which allocation is chosen from the choice set.

In the two-stage decision problem, the decision maker chooses a menu at the ex-ante stage, by anticipating his "social image." This structure is reminiscent of backward induction. The decision maker cares directly about how behavior is perceived by others. The feeling of pride is experienced by resulting altruistic behavior. In the similar way, the feeling of shame is experienced by resulting partially selfish behavior. On the other hand, the decision maker may feel temptation for selfish behavior, irrespective of the publicity of choices.

Revealed Preference Methodology. Suppose that the ex-ante choices are not observed by anyone (see Figure 1). Then, even in the analyst, the ex-ante choices of decision makers are not observed. In principle, however, the axioms can be testable, by collecting anonymous response (ex-post choices) from subjects in experiments. In this sense, the framework of

\footnotetext{
${ }^{6}$ Dana et al. (2006) extend dictator games by introducing the exit option. The choice of exit is not observed by recipients, but an experimenter observes it. The subject does not have the incentive to increase the observer's welfare. Moreover, she does not affect the experimenter's welfare.
} 
preferences over menus is based on the revealed preference methodology.

Notation. We introduce notation for decision-making in social contexts. Let 1 denote the decision maker, and $S$ be the finite set of other agents described by $S=\{2, \cdots n\}$. Let $I=\{1\} \cup S$ be the set of all agents. Let $Z$ be a finite set of outcomes. $\Delta(Z)$ is the set of all lotteries with finite support. For simplicity, we write down $\Delta:=\Delta(Z)$. Let $\Delta^{I}$ be the set of all allocations. The elements of $\Delta^{I}$ are denoted by $\boldsymbol{p}=\left(p_{1}, \cdots, p_{n}\right)=\left(p_{1}, p_{S}\right){ }^{7}$ The lottery $p_{i}$ is an allocation for an agent $i$. Let $\mathcal{A}$ be the set of all non-empty compact subsets of $\Delta^{I}$ endowed with the Hausdorff metric $d_{h}$. The Hausdorff metric is defined by

$$
d_{h}(A, B):=\max \left\{\max _{\boldsymbol{p} \in A} \min _{\boldsymbol{q} \in B} d(\boldsymbol{p}, \boldsymbol{q}), \max _{\boldsymbol{p} \in B} \min _{\boldsymbol{q} \in A} d(\boldsymbol{p}, \boldsymbol{q})\right\},
$$

where $d$ is the Euclidean metric. Menus are denoted by $A, B, C \in \mathcal{A}$.

The primitive of the model is a binary relation $\succeq$ over $\mathcal{A}$. The asymmetric and symmetric parts of $\succeq$ are denoted by $\succ$ and $\sim$, respectively.

Overview. We provide the following axioms. Axiom 1 is a basic requirement to obtain utility representations. In Axiom 2, we require that the decision maker 1 evaluates others' allocations by using her own risk preferences (denoted by $\succsim^{1}$ ). This axiom is also useful to obtain the uniqueness result. Axiom 3 is a standard Pareto condition. Axiom 4 is the key axiom in this paper. We consider image-conscious preferences by inducing the two menu prefreences (denoted by $\succeq_{1}$ and $\succeq_{S}$ ) from the primitive binary relation $\succeq$. In Axiom 5, we require that the subjective state space is finite (see also Kopylov (2009)). Axiom 6 is a weaker version of Independence, and related to the functional forms of image-conscious preferences. Finally, Axiom 7 is also a weaker version of Independence, and requires that the factors of social image concerns are limited to the linear utility representation.

\subsection{Regular Preferences}

First, we provide a basic requirement in decision theory.

Axiom 1. (Standard Preferences): $\succeq$ satisfies completeness, transitivity, continuity, and nondegeneracy.

(i) (Completeness): For any $A, B \in \mathcal{A}, A \succeq B$ or $B \succeq A$.

(ii) (Transitivity): For any $A, B, C \in \mathcal{A}$, if $A \succeq B$ and $B \succeq C$, then $A \succeq C$.

(iii) (Continuity): The sets $\{A \in \mathcal{A} \mid A \succeq B\}$ and $\{A \in \mathcal{A} \mid B \succeq A\}$ are closed (in the Hausdorff metric $d_{h}$ ).

\footnotetext{
${ }^{7}$ This setting is reminiscent of the set-up in Anscombe and Aumann (1963). The allocations in this model are profiles of lotteries. They can be interpreted as the Anscombe=Aumann acts.
} 
(iv) (Non-Degeneracy): There exist $A, B \in \mathcal{A}$ such that $A \succ B$.

Next, we induce two binary relations on $\Delta^{j}(j \in\{1, S\})$, individual preference $\succsim^{1}$ and social preference $\succsim S$. To induce the two binary relations, we focus on singletons. For each $j \in\{1, S\}$, the asymmetric and symmetric parts of $\succsim^{j}$ are denoted by $\succ^{j}$ and $\sim^{j}$, respectively.

Definition 1. $\succsim^{1}$ and $\succsim^{S}$ are defined as follows.

(i) For any $p_{1}, q_{1} \in \Delta, p_{1} \succsim^{1} q_{1}$ if $\left\{\left(p_{1}, r_{S}\right)\right\} \succeq\left\{\left(q_{1}, r_{S}\right)\right\}$ for some $r_{S} \in \Delta^{S}$.

(ii) For any $p_{S}, q_{S} \in \Delta^{S}, p_{S} \succsim^{S} q_{S}$ if $\left\{\left(r_{1}, p_{S}\right)\right\} \succeq\left\{\left(r_{1}, q_{S}\right)\right\}$ for some $r_{1} \in \Delta$.

The first binary relation $\succsim^{1}$ on $\Delta$ is the decision maker 1 's own private ranking over her own allocations. Suppose that two allocations are different from an individual allocation, i.e., $p_{1} \neq q_{1}$, and the allocations for other agents are the same. Then, the decision maker 1's choice should depend on the individual preference.

The next binary relation $\succsim^{S}$ is a social preference relation on $\Delta^{S}$. This is the decision maker 1's preference on other passive agents' allocations. Suppose that two allocations are different from other recipients' allocations, i.e., $p_{S} \neq q_{S}$, and the allocations for her own are the same. Then, the decision maker's choice should depend on the social preference.

Next, we provide a consistency condition between $\succsim^{1}$ and $\succsim^{S}$. This axiom follows from Saito (2015) (p.344).

Axiom 2. (Consistency): For any $p_{S}, q_{S} \in \Delta^{S}$, if $p_{i} \succsim^{1} q_{i}$ for any $i \in S$, then $p_{S} \succsim^{S} q_{S}$.

This axiom states that if all passive recipients' allocations are desirable in terms of the individual ranking, i.e., $\succsim_{1}$, then this ranking holds with the decision maker's social preference. Intuitively speaking, the decision maker 1 may not know about the recipients' preference relations directly. The decision maker 1 evaluates other agents' allocations by using her own private ranking of risk preferences.

The following axiom is a standard Pareto condition.

Axiom 3. (Pareto): The following statements hold.

(i) For any $\boldsymbol{p}, \boldsymbol{q} \in \Delta^{I}$, if $p_{i} \succsim^{1} q_{i}$ and $p_{S} \succsim^{S} q_{S}$, then $\{\boldsymbol{p}\} \succeq\{\boldsymbol{q}\}$.

(ii) For any $\boldsymbol{p}, \boldsymbol{q} \in \Delta^{I}$, if $p_{i} \succ^{1} q_{i}$ and $p_{S} \succ^{S} q_{S}$, then $\{\boldsymbol{p}\} \succ\{\boldsymbol{q}\}$.

Notice that this axiom rules out inequity-averse preferences as studied in Fehr and Schmidt (1999). ${ }^{8}$ Assume that for all $i, j \in S$ with $i \neq j, p_{i}=p_{j}$. If $p_{1}^{\prime} \succ^{1} p_{1}$, then this axiom requires that $\left\{\left(p_{1}^{\prime}, p_{S}\right)\right\} \succ\left\{\left(p_{1}, p_{S}\right)\right\}$. On the other hand, the inequity-averse preference says that there exists a case of $\left\{\left(p_{1}^{\prime}, p_{S}\right)\right\} \prec\left\{\left(p_{1}, p_{S}\right)\right\}$, because of guilt. Since this paper focuses on a foundation for social image concerns, we do not consider the case that (outcome-based) fairness is at play.

\footnotetext{
${ }^{8} \mathrm{I}$ thank an anonymous referee for pointing out this issue. Later, I modify this axiom to capture inequityaverse preferences.
} 


\subsection{Image Concern and Reference-Point Formation}

Before we provide the key axiom, consider the following induced binary relations over menus. $\succeq_{1}$ and $\succeq_{S}$ on $\mathcal{A}$ are defined as follows. For each $j \in\{1, S\}$, the asymmetric and symmetric parts of $\succeq_{j}$ are denoted by $\succ_{j}$ and $\sim_{j}$, respectively.

Definition 2. For each $j \in\{1, S\}$, we say that $A \succeq_{j} B$ if for any $\boldsymbol{p} \in A$ and $\boldsymbol{q} \in B, p_{j} \succsim^{j} q_{j}$.

These induced definitions $\left(\succeq_{1}, \succeq_{S}\right)$ are suitable extensions of individual preference $\succsim^{1}$ and social preference $\succsim^{S}$.

To elicit a reference point endogenously, we introduce a new axiom. The axiom is an application of Disjoint Set-Betweenness introduced in Olszewski (2007).

Axiom 4. (Intrinsic Set-Betweenness): For any $A, B \in \mathcal{A}$ and $j \in\{1, S\}$, if $A \cap B=\varnothing$,

(i) (Temptation-Driven Preference): $A \succeq_{1} B \Rightarrow A \succeq_{1} A \cup B \succeq_{1} B$;

(ii) (Shame-Averse Preference): $A \succeq_{S} B \Rightarrow A \succeq_{S} A \cup B \succeq_{S} B$.

We explain about the key axiom one-by-one. First, Temptation-Driven Preference states that, in terms of self-interested menu-preference $\succeq_{1}$, if a menu $A$ is weakly preferred to another menu $B$, the decision maker exhibits a preference for commitment; that is, the menu $A$ is preferred to the union of the menus $A$ and $B$, i.e., $A \succeq_{1} A \cup B$. This type of commitment is interpreted as temptation-driven preference to act selfishly.

Second, in the similar say, in Shame-Averse Preference, in terms of social interested menu-preference $\succeq_{S}$, if a menu $A$ is weakly preferred to another menu $B$, then the decision maker exhibits a preference for commitment, i.e., $A \succeq_{S} A \cup B$. This type of commitment is interpreted as shame to avoid selfish behavior.

The following axiom is a weaker version of Dominance introduced in Saito (2015).

Axiom 5. (Weak Dominance): For any $A, B \in \mathcal{A}$, if (i) $A \sim_{1} B$, (ii) $A \sim_{S} B$, and (iii) $A \succeq B$, then $A \sim A \cup B$.

The first two conditions state that the menus $A$ and $B$ are indifferent in terms of imageconscious preferences individually and socially. With the third condition, i.e., $A \succeq B$, this axiom requires that the menus $A$ and $A \cup B$ are indifferent. Needless to say, the first two conditions are new. The first condition, $A \sim_{1} B$, says that the level of self-interest in image concerns is the same. In the similar way, the second condition, $A \sim_{S} B$, says that the level of other-regarding attitudes in image concerns is the same. If the conditions hold with $A \succeq B$; that is, the menu $A$ is weakly preferred to the menu $B$, adding the menu $B$ into the menu $A$ does not affect choices from menus. 


\subsection{Relaxing Independence}

We relax the axiom of Independence. Independence is stated as follows. For any $A, B, C \in \mathcal{A}$ and $\lambda \in[0,1]$,

$$
A \succeq B \Rightarrow \lambda A+(1-\lambda) C \succeq \lambda B+(1-\lambda) C .
$$

Since we allow for reference-dependent preferences, the resulting behavior may violate from WARP; that is, irrelevant alternatives may affect decision-making. We weaken Independence by using the induced image-conscious preference relations $\left(\succeq_{1}, \succeq_{S}\right)$.

Axiom 6. (Singleton Independence): For any $A, B \in \mathcal{A}, \boldsymbol{p} \in \Delta^{I}$, and $\lambda \in[0,1]$,

$$
A \succeq B \Rightarrow \lambda A+(1-\lambda)\{\boldsymbol{p}\} \succeq \lambda B+(1-\lambda)\{\boldsymbol{p}\}
$$

There is no reference effect under any mixtures of singleton menus; that is, if the menu $A$ is weakly preferred to the menu $B$, then the ranking does not change after mixing with singletons. This axiom states that Independence holds with the mixture of any singleton menus, since the trade-off under image-conscious preferences does not change after mixing with singletons.

Axiom 7. (Weak Independence): For any $A, B, C \in \mathcal{A}$ and $\lambda \in[0,1]$, if (i) $A \succeq_{1} B$ and (ii) $A \succeq_{S} B$, then

$$
A \succeq B \Rightarrow \lambda A+(1-\lambda) C \succeq \lambda B+(1-\lambda) C .
$$

This axiom is also a weaker version of Independence. The first two conditions are added into the standard version of Independence. Hence, the condition itself is getting stronger. As a result, the axiom itself is getting weaker.

The interpretation of this axiom is as follows. Due to the conditions (i) and (ii), at the ex-ante stage, the decision maker does not feel any trade-off between selfishness and altruism. Under the two conditions, Independence holds; that is, the ranking of menus does not change, since there is no trade-off between the hidden criteria $\left(\succeq_{1}, \succeq_{S}\right)$ on image concerns.

Remark. We consider a relationship between Singleton Independence (Axiom 6) and Weak Independence (Axiom 7). In Axiom 6, arbitrary menus satisfy Singleton Independence; that is, the $\lambda$-mixture with singletons does not change the ranking of menus. On the other hand, in Axiom 7, if a menu $A$ is desirable than another menu $B$ in terms of the induced imageconscious preference relations $\left(\succeq_{1}, \succeq_{S}\right)$, then Independence holds in the standard manner.

\section{Result}

The Model. We investigate the decision maker who faces the two-stage decision problem. The utility of menus is described in the following way. 
Definition 3. $\succeq$ has a reference-dependent image-conscious (RDIC) utilitarian if there is a four-tuple $(u, \boldsymbol{\alpha}, \boldsymbol{\beta}, \boldsymbol{\gamma})$ where $u: \Delta \rightarrow \mathbb{R}$ is a non-constant linear ${ }^{9}$ function, $\boldsymbol{\alpha}:=\left(\alpha_{1},\left(\alpha_{i}\right)_{i \in I}\right)$ is a profile such that $\alpha_{1}>0$, and $\alpha_{i} \geq 0$ for each $i \in S$ with $\sum_{i \in S} \alpha_{i}=1, \boldsymbol{\beta}:=\left(\beta_{1}, \beta_{S}\right)$ is a profile such that $\beta_{1}: u(\Delta)^{2} \rightarrow(-1,+\infty)$ and $\beta_{S}: u(\Delta)^{2} \rightarrow(-\infty, 1)$, and $\gamma$ is a profile such that $\gamma_{j} \in[0,1]$ for each $j \in\{1, S\}$, such that $\succeq$ is represented by a function $V: \mathcal{A} \rightarrow \mathbb{R}$ defined by

$$
\begin{aligned}
V(A)=\max _{\boldsymbol{p} \in A}\left[\sum_{i \in I} \alpha_{i} u\left(p_{i}\right)\right. & +\beta_{1}\left(\overline{\boldsymbol{u}}_{A}\right)\left(\alpha_{1}\left(u\left(p_{1}\right)-\bar{u}_{1}(A)\right)\right) \\
& \left.-\beta_{S}\left(\overline{\boldsymbol{u}}_{A}\right)\left(\sum_{i \in S} \alpha_{i} u\left(p_{i}\right)-\bar{u}_{S}(A)\right)\right],
\end{aligned}
$$

where $\overline{\boldsymbol{u}}_{A}:=\left(\bar{u}_{1}(A), \bar{u}_{S}(A)\right)$ is a reference point of the menu $A$ defined by

$$
\bar{u}_{1}(A)=\gamma_{1} \max _{\boldsymbol{q} \in A} u\left(q_{1}\right)+\left(1-\gamma_{1}\right) \min _{\boldsymbol{r} \in A} u\left(r_{1}\right),
$$

and

$$
\bar{u}_{S}(A)=\gamma_{S} \max _{\boldsymbol{q} \in A} \sum_{i \in S} \alpha_{i} u\left(q_{i}\right)+\left(1-\gamma_{S}\right) \min _{\boldsymbol{r} \in A} \sum_{i \in S} \alpha_{i} u\left(r_{i}\right)
$$

The model has the following building blocks $(u, \boldsymbol{\alpha}, \boldsymbol{\beta}, \boldsymbol{\gamma})$. The first component $u$ is a self-utility that evaluates allocations. Since the decision maker does not know other agents' preferences, she evaluates others' allocations by using her risk preference, i.e., her utility function.

\subsection{Representation Theorem}

We state the main result.

Theorem 1. The following statements are equivalent:

(a) $\succeq$ satisfies Axioms 1 - 7 .

(b) $\succeq$ has a reference-dependent image-conscious utilitarian.

\section{The Building Blocks of the Model}

We explain about the model in detail. $\succeq$ satisfies the axioms in Theorem 1 if and only if there exists a four-tuple $(u, \boldsymbol{\alpha}, \boldsymbol{\beta}, \boldsymbol{\gamma})$ where

(i) $u: \Delta \rightarrow \mathbb{R}$ is a non-constant linear function (self-utility);

(ii) $\boldsymbol{\alpha}:=\left(\alpha_{1},\left(\alpha_{i}\right)_{i \in I}\right)$ is a profile such that $\alpha_{1}>0$, and $\alpha_{i} \geq 0$ for each $i \in S$ with $\sum_{i \in S} \alpha_{i}=1$;

\footnotetext{
${ }^{9}$ The definition of linearity is as follows. We say that a function $u: \Delta \rightarrow \mathbb{R}$ is linear if for any $p, q \in \Delta$ and $\lambda \in(0,1), u(\lambda p+(1-\lambda) q)=\lambda u(p)+(1-\lambda) u(q)$.
} 
(iii) $\boldsymbol{\beta}:=\left(\beta_{1}, \beta_{S}\right)$ is a profile such that $\beta_{1}: u(\Delta)^{2} \rightarrow(-1,+\infty)$ and $\beta_{S}: u(\Delta)^{2} \rightarrow(-\infty, 1)$;

(iv) $\gamma:=\left(\gamma_{1}, \gamma_{S}\right)$ is a profile such that $\gamma_{j} \in[0,1]$ for each $j \in\{1, S\}$.

The decision maker chooses the best allocation from menus, by taking into account of the three terms. The self-utility $u$ is an expected utility that captures evaluations of allocations for each agent (risk preferences). The parameter $\boldsymbol{\alpha}$ captures the attitude toward pure altruism and selfishness. The parameter $\boldsymbol{\beta}$ captures social emotions concerning social image. The parameter $\gamma$ is a belief on the criterion of reference-point formation.

Outcome-Based Utility. The first term captures the outcome-based utility (of allocations). The first term is evaluated without reference-dependence. We can write down the first term by

$$
\alpha_{1} u\left(p_{1}\right)+\sum_{i \in S} \alpha_{i} u\left(p_{i}\right) .
$$

The component $\boldsymbol{\alpha}$ is a profile of $\alpha_{1}$ and $\alpha_{S}:=\left(\alpha_{i}\right)_{i \in S}$ where $\alpha_{1}$ captures the level of pure altruism, and $\alpha_{S}$ captures other-regarding preferences including inequity aversion. ${ }^{10}$ Since $\sum_{i \in S} \alpha_{i}=1, \frac{\sum_{i \in S} \alpha_{i}}{\alpha_{1}}=\frac{1}{\alpha_{1}}$ is the relative weight on the level of pure altruism and selfishness. As $\alpha_{1}$ is getting smaller, the decision maker is willing to increase the other agents' allocation $p_{S}$ (see the comparative statics in Proposition 2). In Section 4, we modify the axioms in Theorem 1, and study inequity-averse preferences.

Social Image Concerns and Reference Point Formation. The component $\boldsymbol{\beta}$ is a profile of $\beta_{1}$ and $\beta_{S}$ where both $\beta_{1}$ and $\beta_{S}$ are relative weights on social emotions. The component $\gamma$ is a profile of $\gamma_{1}$ and $\gamma_{S}$ where $\gamma_{1}$ controls the reference level of the decision maker's own allocation, and $\gamma_{S}$ controls the reference level of the others' allocations.

Remark. In the existing studies, reference points are fixed. The level of social emotions does not change, even if the level of social pressure arising from menus changes. This paper generalizes the point, by allowing for context-dependence, in particular, reference-dependence.

Mixing Best and Worst. As mentioned in Introduction, the existing studies sit the reference points, the most peronally normative allocation (Dillenberger and Sadowski, 2012), the most selfish allocation (Evren and Minardi, 2017), or both (Saito, 2015). The maximal elements are reference points, and deviating from them can lead to social emotions like

\footnotetext{
${ }^{10}$ The main difference between this paper and the model of inequity aversion like Fehr and Schmidt (1999) is as follows. In inequity aversion, social preferences are captured by outcome-based utility, which means that fairness is considered by the difference of payoffs between the decision maker and other agents. See Rohde (2010) for the axiomatization of the Fehr and Schmidt (1999). Hashidate (2019) presents an axiomatic study on a general class of interdependent preferences.
} 
shame (Dillenberger and Sadowski, 2012), warm-glow (Evren and Minardi, 2017), and pride, temptation, and shame (Saito, 2015).

Caring about the worst-off position is supported by Kameda et al. (2016), the study on social distribution for others. ${ }^{11}$ They argue that the "max-min" concern (maximizing the minimum possible payoff) operates as a strong cognitive anchor in both distributive decisions for others and economic decisions for oneself. Although social image concerns is different from Kameda et al. (2016), we follow their study in that caring the worst-off position impacts social-image formation. ${ }^{12}$

Following Kameda et al. (2016), we consider not only the maximal element, but also the minimal element in menus. Hershey and Schoemaker (1985) found that when asked for the probability $p$ that made them indifferent between outcome $z$ and a lottery $\left(p, x_{1} ; 1-p, x_{2}\right)$ with $x_{1}>z>x_{2}$. Their subjects took $z$ as their reference point. ${ }^{13}$

In the RDIC utilitarian, $\gamma_{j}(j \in\{1, S\})$ is similar to the finding in Hershey and Schoemaker (1985). Take an arbitrary menu $A \in \mathcal{A}$. Consider $j=1$. The decision maker considers the level of $\gamma_{1}$ that makes the reference point $\bar{u}_{1}(A)$ and a lottery $\left(\gamma_{1}, \max _{\boldsymbol{p} \in A} u\left(p_{1}\right) ; 1-\right.$ $\left.\gamma_{1}, \min _{\boldsymbol{p} \in A} u\left(p_{1}\right)\right)$ indifferent. ${ }^{14}$ Deviating from our reference points can capture not only pride, temptation, warm-glow, and shame, but also spitefulness, and regret.

\section{Ex-Ante Gain-Loss Utility and Social Emotions}

In the RDIC utilitarian, the pair of $\boldsymbol{\gamma}$ and $\boldsymbol{\beta}$ determines social emotions, which are summarized by Table 1 and Table 2 . Let $j \in\{1, S\}$. Given a menu $A \in \mathcal{A}$, let $\Delta_{j}:=u\left(p_{j}\right)-\bar{u}_{j}(A)$ where $\boldsymbol{p} \in A$ is the maximizer of the menu $A$.

the Case: $\Delta_{1}>0$ and $-1<\beta_{1}(\cdot)<0$. This case corresponds to shame of acting selfishly. The term $\Delta_{1}>0$ is the joy (pleasure) of taking. The parameter $-1<\beta_{1}(\cdot)<0$ means that the decision maker deliberately decreases the relation weight on his own payoff. Such a pair $\left(\Delta_{1}, \beta_{1}\right)$ captures shame because the second term is non-positive. This term implies that the second term is the psychological cost on acting selfishly.

\footnotetext{
${ }^{11}$ The robust concern about the minimum outcomes was correlated with activation of the right temporoparietal junction $(R T P J)$, the region associated with perspective taking.

${ }^{12}$ In a finite setting, Doron and Steverson (2018) study the case of bad temptation, and characterize the model of bad temptation where the decision maker's temptation ranking is the opposite of her commitment preference. This paper considers image-conscious preference by considering temptation-driven preference.

${ }^{13}$ Baillon et al. (2018) explores experimentally how people form their reference points in decision-making under risk. Their result indicates that two reference-point rules stand out. One is the status-quo rule, the other is a max-min rule, a security-based rule based on Hershey and Schoemaker (1985).

${ }^{14}$ In the behavioral economics literature, Köszegi and Szeidl (2012) and Bushong et al. (2017) study a multiattribute utility representation in that the weight on each attribute depends on the ranges of choice-sets. In the similar way, this paper studies a decision maker who cares about both the maximal and the worst values in choice sets for social image concerns.
} 
Table 1: Social Image with $\beta_{1}$

\begin{tabular}{|c|c|c|}
\hline & $-1<\beta_{1}(\cdot)<0$ & $0 \leq \beta_{1}(\cdot)<+\infty$ \\
\hline$\Delta_{1}>0$ & shame & temptation \\
\hline$\Delta_{1}<0$ & pride & regret \\
\hline
\end{tabular}

the Case: $\Delta_{1}>0$ and $0 \leq \beta_{1}(\cdot)<+\infty$. This case corresponds to temptation to act selfishly. The term $\Delta_{1}>0$ is the joy (pleasure) of taking. The parameter $0 \leq \beta_{1}(\cdot)<+\infty$ means that the decision maker increases the relative weight on his own payoff. Such a pair $\left(\Delta_{1}, \beta_{1}\right)$ captures temptation because the second term is non-negative. This way of the description on temptation is different from the exisiting studies like Gul and Pesendorfer (2001). This term implies that increasing his own payoff leads to the improvement of his ex-ante utility.

the Case: $\Delta_{1}<0$ and $-1<\beta_{1}(\cdot)<0$. This case corresponds to pride of acting altruistically. The term $\Delta_{1}<0$ is the unpleasure of taking. The parameter $-1<\beta_{1}(\cdot)<0$ means that the decision maker deliberately decreases the relation weight on his own payoff. Such a pair $\left(\Delta_{1}, \beta_{1}\right)$ captures pride because the second term is non-negative. This term implies that the ex-ante utility increases as the decision maker deliberately decreases his own payoff.

the Case: $\Delta_{1}<0$ and $0 \leq \beta_{1}(\cdot)<+\infty$. This case corresponds to regret of act selfishly. The term $\Delta_{1}<0$ is the unpleasure of taking. The parameter $0 \leq \beta_{1}(\cdot)<+\infty$ means that the decision maker increases the relative weight on his own payoff. Such a pair $\left(\Delta_{1}, \beta_{1}\right)$ captures regret on acting altruistically because the second term is non-positive. This term implies that the second term is the psychological cost on altruistic behavior as regret.

Table 2: Social Image with $\beta_{S}$

\begin{tabular}{|c|c|c|}
\hline & $-\infty<\beta_{S}(\cdot) \leq 0$ & $0<\beta_{S}(\cdot)<1$ \\
\hline$\Delta_{S}>0$ & warm-glow & regret \\
\hline$\Delta_{S}<0$ & shame & spitefulness \\
\hline
\end{tabular}

the Case: $\Delta_{S}>0$ and $-\infty<\beta_{S}(\cdot) \leq 0$. This case corresponds to warm-glow giving. The term $\Delta_{S}>0$ is the joy (pleasure) of giving. The parameter $-\infty<\beta_{S}(\cdot) \leq 0$ means that the decision maker deliberately increases the relation weight on others' payoff. Such a pair $\left(\Delta_{S}, \beta_{S}\right)$ captures warm-glow because the third term is non-negative. This term implies that the third term is the psychological benefit on acting altruistically. 
the Case: $\Delta_{S}>0$ and $0<\beta_{S}(\cdot)<1$. This case corresponds to regret of acting altruistically. The term $\Delta_{S}>0$ is the joy (pleasure) of giving. The parameter $-0<\beta_{S}(\cdot)<1$ means that the decision maker deliberately decreases the relation weight on others' payoff. Such a pair $\left(\Delta_{S}, \beta_{S}\right)$ captures regret on acting altruistically because the third term is non-positive. This term implies that the third term is the psychological cost on acting altruistically.

the Case: $\Delta_{S}<0$ and $-\infty<\beta_{S}(\cdot) \leq 0$. This case corresponds to shame of acting selfishly. The term $\Delta_{S}<0$ is the unpleasure of giving. The parameter $-\infty<\beta_{S}(\cdot) \leq 0$ means that the decision maker deliberately increases the relation weight on others' payoff. Such a pair $\left(\Delta_{S}, \beta_{S}\right)$ captures shame because the third term is non-negative. This term implies that the third term is the psychological cost on acting selfishly; that is, the decision maker chooses the allocation that is below his intrinsic reference point on others.

the Case: $\Delta_{S}<0$ and $0<\beta_{S}(\cdot)<1$. This case corresponds to spitefulness to act selfishly. The term $\Delta_{S}<0$ is the unpleasure of giving. The parameter $-0<\beta_{S}(\cdot)<1$ means that the decision maker deliberately decreases the relation weight on others' payoff. Such a pair $\left(\Delta_{S}, \beta_{S}\right)$ captures spitefulness because the third term is non-negative. This term implies that the ex-ante utility is beneficial due to the selfish act such that the chosen allocation is below his intrinsic reference point on others.

\section{Ex-Post Utility}

The ex-post utility $U: \Delta^{I} \rightarrow \mathbb{R}$ (see Figure 1) is described by

$$
U(\boldsymbol{p})=\left(1+\beta_{1}\left(\overline{\boldsymbol{u}}_{A}\right)\right) \alpha_{1} u\left(p_{1}\right)+\left(1-\beta_{S}\left(\overline{\boldsymbol{u}}_{A}\right)\right) \sum_{i \in S} \alpha_{i} u\left(p_{i}\right) .
$$

Since $\beta_{1}: u(\Delta)^{2} \rightarrow(-1,+\infty)$ and $\beta_{S}: u(\Delta)^{2} \rightarrow(-\infty, 1)$, the actual utility at the ex-post stage is monotone. The ex-post utility can be regarded as a relative/weighted utilitarian, in which the weights depend on social image concerns, and the weights are reference-dependent.

We explain about the effect of social image. First, consider the parameter $\beta_{1}$. On the one hand, in the case of $-1<\beta_{1} \leq 0$, the decision maker deliberately decreases the overall weight on her own allocations, due to pride or shame. On the other hand, in the case of $\beta_{1}>0$, the overall weight on her own allocations gets high, due to temptation or regret.

Second, consider the parameter $\beta_{S}$. On the one hand, in the case of $\beta \leq 0$, the decision maker feels warm-glow or shame, which increases the relative weight on others' allocations. On the other hand, in the case of $0<\beta_{S}<1$, the decision maker feels regret or spitefulness. The overall weight on others' allocations decreases. 


\subsection{Uniqueness Result}

We state the uniqueness result.

Proposition 1. Suppose that the two representations $(u, \boldsymbol{\alpha}, \boldsymbol{\beta}, \boldsymbol{\gamma})$ and $\left(u^{\prime}, \boldsymbol{\alpha}^{\prime}, \boldsymbol{\beta}^{\prime}, \boldsymbol{\gamma}^{\prime}\right)$ represent the same binary relation $\succeq$. Then, the following statements hold:

(i) $u^{\prime}=a u+b$ for some $a>0$ and $b \in \mathbb{R}$;

(ii) $\boldsymbol{\alpha}=\boldsymbol{\alpha}^{\prime}$;

(iii) $\boldsymbol{\beta}=\boldsymbol{\beta}^{\prime}$;

(iv) $\gamma=\gamma^{\prime}$.

The uniqueness result mainly follows from Axiom 2 (Consistency). Because of this axiom, the decision maker evaluates allocations by using one self-utility function $u$. $u$ is an expected utility. $\boldsymbol{\alpha}$ is uniquely identified. By applying the result in Olszewski (2007), $\boldsymbol{\gamma}$ is uniquely identified. Notice that the parameter for social image $\boldsymbol{\beta}$ depends on reference points. Given menus, $\boldsymbol{\beta}$ is uniquely identified.

\section{The Effects on Social Emotions}

\subsection{Altruism, Selfishness and Inequity Aversion}

We study the effect on the parameter $\boldsymbol{\alpha}$. The component $\alpha_{1}$ captures the level of pure altruism/selfishness. The component $\alpha_{S}=\left(\alpha_{i}\right)_{i \in S}$ captures the level of other-regarding preferences. First, by modifying the axioms in Theorem 1, we study inequity-averse preferences. Second, we study a comparative attitude toward pure altruism/selfishness.

\section{Pure Altruism and Inequity Aversion}

To capture inequity aversion, we modify the axioms in Theorem 1. To do so, first, we modify Axiom 3 (Pareto). Instead, we introduce the property of monotonicity with respect to equal allocations. Second, we strengthen Axiom 5 (blue Weak Dominance). In fact, we require that $\succeq$ satisfies Strategic Rationality in Kreps (1979); We study the case that there is no effect on social image concerns. Third, we relax Independence to study inequity-averse preferences.

We present a brief guideline of the axiomatization. First, we impose on Strategic Rationality instead of Weak Dominance to capture the role of inequity aversion explicitly. Second, we relax Pareto and Independence to study Inequity Aversion. Finally, we provide the axiom on Inequity Aversion.

The axiom of Strategic Rationality introduced in Kreps (1979), is stated in the following way. This axiom states that the decision maker exhibits neither preferences for flexibility nor preferences for commitment, which captures social emotions in social contexts. 
Axiom 8. (Strategic Rationality): For any $A, B \in \mathcal{A}, A \succeq B \Rightarrow A \sim A \cup B$.

We obtain the following result. This is the case that there is no effect on social image concerns. The corollary states that social image concerns are axiomatically different from inequity aversion.

Corollary 1. Suppose that $\succeq$ is represented by a RDIC with a four-tuple $(u, \boldsymbol{\alpha}, \boldsymbol{\beta}, \boldsymbol{\gamma})$. Then, $\succeq$ satisfies Axiom 8 if and only if for any $A \in \mathcal{A}, \beta_{1}\left(\overline{\boldsymbol{u}}_{A}\right)=0$ and $\beta_{S}\left(\overline{\boldsymbol{u}}_{A}\right)=0$.

Next, we relax Axiom 3 (Pareto). The Pareto condition is not consistent with inequityaverse preferences. The following weak monotone condition says that fairness is sustained in the case that every agent has the same allocation.

Axiom 9. (Monotonicity with respect to Equal Allocation): For any $p, q \in \Delta$, if $p \succ^{i} q$, then $\{(p, \cdots, p)\} \succeq\{(q, \cdots, q)\}$.

We relax the Independence axiom that is related to Axiom 6 and Axiom 7 in this paper. We define comonotonic allocations.

Definition 4. For any $\boldsymbol{p}, \boldsymbol{q} \in \Delta^{I}$, two allocations $\boldsymbol{p}$ and $\boldsymbol{q}$ are comonotonic if there exists no $i \in S$ such that $p_{i} \succ^{1} p_{1}$ and $q_{i} \prec^{1} q_{1}$.

The following axiom is introduced in Schmeidler (1989). This axiom states that if two allocations are comonotonic, then $\lambda$-mixture $(\lambda \in(0,1))$ with comonotonic allocations does not change the ranking of allocations.

Axiom 10. (Comonotonic Independence): For any $\boldsymbol{p}, \boldsymbol{q}, \boldsymbol{r} \in \Delta^{I}$ that are pairwise comonotonic, and $\lambda \in(0,1)$,

$$
\{\boldsymbol{p}\} \succeq\{\boldsymbol{q}\} \Rightarrow \lambda\{\boldsymbol{p}\}+(1-\lambda)\{\boldsymbol{r}\} \succeq \lambda\{\boldsymbol{q}\}+(1-\lambda)\{\boldsymbol{r}\}
$$

We provide an axiom of Inequity Aversion, and obtain the result.

Axiom 11. (Inequity Aversion): $\succsim$ satisfies (i) envy and (ii) guilt: For any $p, p^{\prime}, p^{\prime \prime} \in \Delta$ with $p^{\prime} \succ^{1} p \succ^{1} p^{\prime \prime}$

(i) (Envy): $\left\{\left(p,(p)_{i \in S}\right)\right\} \succeq\left\{\left(p,\left(p^{\prime}\right)_{i \in S}\right)\right\}$;

(ii) (Guilt): $\left\{\left(p,(p)_{i \in S}\right)\right\} \succeq\left\{\left(p,\left(p^{\prime \prime}\right)_{i \in S}\right)\right\}$.

Corollary 2. $\succeq$ satisfies Axioms 1, 8, 9, 10, and 11 if and only if there exists a tuple $\left(u, \alpha_{1},\left(\alpha_{\text {envy }}^{i}, \alpha_{\text {guilt }}^{i}\right)_{i \in S}\right)$ where $u: \Delta \rightarrow \mathbb{R}$ is a non-constant function, $\alpha_{1}>0$, and for each $i \in S, \alpha_{\text {envy }}^{i} \geq 0, \alpha_{\text {guilt }}^{i} \geq 0$, such that $\succeq$ is represented by

$$
V(A)=\max _{p \in A}\left[\alpha_{1} u\left(p_{1}\right)-\sum_{i \in S}\left(\alpha_{\text {envy }}^{i} \max \left\{u\left(p_{i}\right)-u\left(p_{1}\right), 0\right\}+\alpha_{\text {guilt }}^{i} \max \left\{u\left(p_{1}\right)-u\left(p_{i}\right), 0\right\}\right)\right] .
$$


The framework of preferences over menus identifies image conscious preferences like pride, shame, and temptation. On the other hand, inequity-averse preferences are identified by preferences over singletons, i.e., preference over allocations. The main finding is that we can identify image conscious preferences and inequity-averse preference separately. ${ }^{15}$

\section{Comparative Statics on Pure Altruism}

We study the comparative statics on pure altruism, captured by the parameter $\alpha_{1}$ in the model. ${ }^{16} \alpha_{1}$ is the level of pure selfishness. The attitude toward pure altruism is captured by singleton menus, i.e., outcome-based utility.

Consider two decision makers $X$ and $Y$. In this paper, both Mr. $X$ and Mr. $Y$ are denoted by a decision maker 1 . Assume that the set of other agents $S$ is fixed.

Definition 5. For any $\succeq^{X}$ and $\succeq^{Y}$ on $\mathcal{A}$ such that $\succsim^{X_{j}}=\succsim^{Y_{j}}$ for each $j \in\{1, S\}, \succeq^{X}$ is more purely altruistic than $\succeq^{Y}$ if, for any $\boldsymbol{p}, \boldsymbol{q} \in \Delta^{I}$ with $p_{S} \succ_{S}^{h} q_{S}$ for each $h \in\{X, Y\}$,

$$
\{\boldsymbol{p}\} \succeq^{Y}\{\boldsymbol{q}\} \Rightarrow\{\boldsymbol{p}\} \succeq^{X}\{\boldsymbol{q}\}
$$

We consider the case that an allocation $\boldsymbol{p}$ is superior to $\boldsymbol{q}$ in the social preference: $p_{S} \succ_{S}^{h} q_{S}$ for each $h \in\{X, Y\}$. Under the assumption that $\succsim^{X_{j}}=\succsim^{Y_{j}}$ for each $j \in\{1, S\}$, the definition says that if Mr. $Y$ weakly prefers $\boldsymbol{p}$ to $\boldsymbol{q}$ with $p_{S} \succ_{S}^{Y} q_{S}$, then another decision maker Mr. $X$ also weakly prefers $\boldsymbol{p}$ to $\boldsymbol{q}$ with $p_{S} \succ^{X_{S}} q_{S}$.

We obtain the following result.

Proposition 2. Suppose that for each $h \in\{X, Y\}, \succeq_{h}$ is represented by a four-tuple $\left(u, \boldsymbol{\alpha}^{h}, \boldsymbol{\beta}, \gamma\right)$. Then, $X$ is more purely altruistic than $Y$ if and only if $\alpha_{1}^{X} \leq \alpha_{1}^{Y}$.

As $\alpha_{1}$ is getting smaller, the resulting behavior is altruistic (see Figure 2; the case of $0<\alpha<1)$. In the RDIC representation, the case of $\alpha_{1}=1$ corresponds to egalitarian.

\subsection{Pride and Temptation}

We study the role of social emotions like pride, warm-glow, and temptation, respectively. We characterize the parameter $\beta_{1}$ that represents pride $\left(-1<\beta_{1}\left(u(\Delta)^{2}\right) \leq 0\right)$ and temptation $\left(0<\beta_{1}\left(u(\Delta)^{2}\right)<+\infty\right)$.

To focus on the effect on pride, we provide the following axiom. We rule out the effect on shame. The axiom is a weaker version of Axiom 5 (Weak Dominance).

Axiom 12. (Pride-Based Dominance): For any $A, B \in \mathcal{A}$, if (i) $A \sim_{1} B$ and (ii) $A \succeq B$, then $A \sim A \cup B$.

\footnotetext{
${ }^{15}$ Dillenberger and Sadowski (2012) identifies shame, by using a personal norm utility that is more altruistic than a self-utility $u$. The personal norm may include inequity aversion.

${ }^{16}$ I am grateful to Youichiro Higashi for his helpful comment.
} 


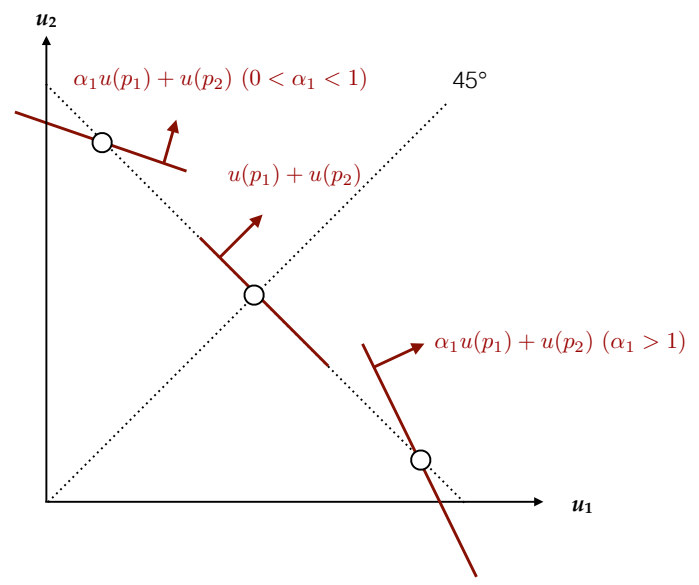

Figure 2: Comparative Statics on Pure Altruism

We study the case that the decision maker purely has the freedom to behave selfishly $\left(\gamma_{1}=1\right)$. To study a relationship between prosocial behavior and the freedom to be selfish, we consider the following axiom.

Axiom 13. (Self-Interest): For any $A, B \in \mathcal{A}, A \succeq_{1} B \Rightarrow A \sim_{1} A \cup B$.

The axiom states that if a menu $A$ is weakly preferred to another menu $B$ in terms of individual menu-preferences, then the desirable menu $A$ and the union of them $A \cup B$ are indifferent. At the ex-ante stage, in terms of self-evaluation of allocations, the decision maker predicts that adding $B$ into $A$ does not have any effect on resulting behavior at the ex-post stage.

Corollary 3. Suppose that $\succeq$ is represented by a RDIC with a four-tuple $(u, \boldsymbol{\alpha}, \boldsymbol{\beta}, \boldsymbol{\gamma})$. Then, $\succeq_{1}$ exhibits Self-Interest Dominance if and only if $\gamma_{1}=1$.

The Corollary 3 states that if $\succeq$ satisfies Axiom 13 (Self-Interest), the most selfish allocation in choice sets is the reference point.

\section{Pride}

We provide the axiom that identifies the case that the decision maker exhibits pride $(-1<$ $\left.\beta_{1}\left(u(\Delta)^{2}\right) \leq 0\right)$. Fix the doubleton $\{\boldsymbol{p}, \boldsymbol{q}\}$, and suppose that the following conditions hold:

(i) $\{\boldsymbol{q}\} \succ_{1}\{\boldsymbol{p}\}$;

(ii) $\{\boldsymbol{p}\} \succ_{S}\{\boldsymbol{q}\}$; and 
(iii) $\{\boldsymbol{p}, \boldsymbol{q}\} \succ\{\boldsymbol{q}\}$.

The first condition states that $\boldsymbol{q}$ is self-interestedly superior to $\boldsymbol{p}$. On the contrary, the second condition states that $\boldsymbol{p}$ is social-interestedly superior to $\boldsymbol{q}$. The third condition states that $\boldsymbol{p}$ is chosen from $\{\boldsymbol{p}, \boldsymbol{q}\}$; that is, it is inferred that $\boldsymbol{p}$ is better than $\boldsymbol{q}$ at the ex-post stage. We call the doubleton $\{\boldsymbol{p}, \boldsymbol{q}\}$ socially conflicting if the three conditions hold.

Axiom 14. (Pride): For any $\boldsymbol{p}, \boldsymbol{q} \in \Delta^{I}$ such that is $\{\boldsymbol{p}, \boldsymbol{q}\}$ is a socially conflicting menu,

$$
\{\boldsymbol{p}, \boldsymbol{q}\} \succeq\{\boldsymbol{p}\}
$$

Proposition 3. Suppose that $\succeq$ satisfying Axiom 12 (Pride-Based Dominance) and Axiom 13 (Self-Interest) is represented by a RDIC with a four-tuple $(u, \boldsymbol{\alpha}, \boldsymbol{\beta}, \boldsymbol{\gamma})$ with $\beta_{S}\left(u(\Delta)^{2}\right)=0$ and $\gamma_{1}=1 . \succeq$ exhibits Pride if and only if $-1<\beta_{1}\left(u(\Delta)^{2}\right) \leq 0$.

\section{Comparative Statics on Pride}

We investigate the comparative attitude toward pride. In the same way as the study of the comparative statics on $\alpha$, consider two decision makers, Mr.X and Mr.Y, who are acted as a decision maker 1. Assume that the set of other agents $S$ is fixed. Let $\succeq^{X}$ and $\succeq^{Y}$ be the two decision maker's preferences over menus.

Definition 6. $\succeq^{X}$ is more pride-seeking than $\succeq^{Y}$ if the following holds; for any $\boldsymbol{p}, \boldsymbol{q} \in \Delta^{I}$ such that is $\{\boldsymbol{p}, \boldsymbol{q}\}$ is a socially conflicting menu, and $r \in \Delta$,

$$
\{\boldsymbol{p}, \boldsymbol{q}\} \succeq^{Y}\{(r, \cdots, r)\} \Rightarrow\{\boldsymbol{p}, \boldsymbol{q}\} \succeq^{X}\{(r, \cdots, r)\}
$$

Remember that firstly $\boldsymbol{p}$ and $\boldsymbol{q}$ are conflicting. $\boldsymbol{q}$ is self-interestedlly preferred, but $\boldsymbol{p}$ is preferred at the ex-post stage. Thus, the decision maker would feel pride, by choosing $\boldsymbol{p}$. Second, a singleton $\{(r, \cdots, r)\}$ does not cause any psychological effect including inequity aversion. Hence, $\{\boldsymbol{p}, \boldsymbol{q}\} \succeq\{(r, \cdots, r)\}$ stems from pride.

Proposition 4. Suppose that $\succeq^{j}$ is represented by a RDIC with a four-tuple $\left(u, \boldsymbol{\alpha}, \boldsymbol{\beta}^{j}, \boldsymbol{\gamma}\right)$ where $j \in\{X, Y\}$. Then, $\succeq^{X}$ is more pride-seeking than $\succeq^{Y}$ if and only if $-1<\beta_{1}^{X}\left(u(\Delta)^{2}\right)<$ $\beta_{1}^{Y}\left(u(\Delta)^{2}\right) \leq 0$.

\section{Temptation}

We provide the axiom that identifies the case that the decision maker exhibits temptation $\left(0<\beta_{1}\left(u(\Delta)^{2}\right)<+\infty\right)$. Fix the doubleton $\{\boldsymbol{p}, \boldsymbol{q}\}$, and suppose that the following conditions hold:

(i) $\{\boldsymbol{q}\} \succ_{1}\{\boldsymbol{p}\}$; 
(ii) $\{\boldsymbol{p}\} \succ_{S}\{\boldsymbol{q}\}$; and

(iii) $\{\boldsymbol{q}\} \succ\{\boldsymbol{p}, \boldsymbol{q}\}$.

The first condition states that $\boldsymbol{q}$ is self-interestedly superior to $\boldsymbol{p}$. On the contrary, the second condition states that $\boldsymbol{p}$ is social-interestedly superior to $\boldsymbol{q}$. The third condition states that $\boldsymbol{p}$ is chosen from $\{\boldsymbol{p}, \boldsymbol{q}\}$; that is, it is inferred that $\boldsymbol{q}$ is better than $\boldsymbol{p}$ at the ex-post stage. We call the doubleton $\{\boldsymbol{p}, \boldsymbol{q}\}$ selfishly conflicting if the three conditions hold.

Axiom 15. (Temptation): For any $\boldsymbol{p}, \boldsymbol{q} \in \Delta^{I}$ such that is $\{\boldsymbol{p}, \boldsymbol{q}\}$ is a selfishly conflicting menu,

$$
\{\boldsymbol{p}, \boldsymbol{q}\} \succeq\{\boldsymbol{p}\}
$$

Proposition 5. Suppose that $\succeq$ satisfying Axiom 12 (Pride-Based Dominance) is represented by a RDIC with a four-tuple $(u, \boldsymbol{\alpha}, \boldsymbol{\beta}, \boldsymbol{\gamma})$ with $\beta_{S}\left(u(\Delta)^{2}\right)=0$. $\succeq$ exhibits Temptation if and only if $0<\beta_{1}\left(u(\Delta)^{2}\right)<+\infty$.

\section{Comparative Statics}

We investigate the comparative attitude toward temptation. Consider two decision makers, Mr. $X$ and Mr.Y, who are acted as a decision maker 1. Assume that the set of other agents $S$ is fixed. Let $\succeq^{X}$ and $\succeq^{Y}$ be the two decision maker's preferences over menus.

Definition 7. $\succeq^{X}$ is more pride-seeking than $\succeq^{Y}$ if the following holds; for any $\boldsymbol{p}, \boldsymbol{q} \in \Delta^{I}$ such that is $\{\boldsymbol{p}, \boldsymbol{q}\}$ is a selfishly conflicting menu, and $r \in \Delta$,

$$
\{\boldsymbol{p}, \boldsymbol{q}\} \succeq^{Y}\{(r, \cdots, r)\} \Rightarrow\{\boldsymbol{p}, \boldsymbol{q}\} \succeq^{X}\{(r, \cdots, r)\}
$$

First, $\boldsymbol{p}$ and $\boldsymbol{q}$ are conflicting. $\boldsymbol{q}$ is self-interestedlly preferred, and $\boldsymbol{p}$ is preferred at the expost stage. Second, a singleton $\{(r, \cdots, r)\}$ does not cause any psychological effect including inequity aversion. Hence, $\{\boldsymbol{p}, \boldsymbol{q}\} \succeq\{(r, \cdots, r)$ stems from temptation.

Proposition 6. Suppose that $\succeq^{j}$ is represented by a RDIC with a four-tuple $\left(u, \boldsymbol{\alpha}, \boldsymbol{\beta}^{j}, \boldsymbol{\gamma}\right)$ where $j \in\{X, Y\}$. Then, $\succeq^{X}$ is more temptation-driven than $\succeq^{Y}$ if and only if $0<\beta_{1}^{Y}\left(u(\Delta)^{2}\right)<$ $\beta_{1}^{X}\left(u(\Delta)^{2}\right)<+\infty$.

\section{Overwhelming Temptation}

We consider the case of overwhelming temptation. The decision maker is purely self-interested. Such a decision maker only maximizes his own allocation. This is one of the standard assumptions in economic theory. In this sense, a type of self-interested decision makers is a special case of the RDIC representation. The case corresponds to overwhelming temptation $\left(\beta_{1}(\cdot) \rightarrow+\infty\right)$. 


\subsection{Shame}

We study the role of shame $\left(-1 \leq \beta_{S}(\cdot) \leq 0\right)$. We provide the axiom that identifies the case that the decision maker exhibits shame.

\section{Shame}

Fix a menu $\{\boldsymbol{p}, \boldsymbol{q}\}$, and take an arbitrary allocation $\boldsymbol{r} \in \Delta^{I}$. Suppose the following conditions hold:

1. $\{\boldsymbol{r}\} \succ_{1}\{\boldsymbol{p}\}$

2. $\{\boldsymbol{q}\} \succ_{S}\{\boldsymbol{p}\}$; and

3. $\{\boldsymbol{p}, \boldsymbol{q}, \boldsymbol{r}\} \succ\{\boldsymbol{q}, \boldsymbol{r}\}$.

The first condition states that $\boldsymbol{r}$ is individually optimal, and $\boldsymbol{p}$ is not individually optimal. The second condition states that $\boldsymbol{q}$ is socially superior to $\boldsymbol{p}$. The third condition states that $\boldsymbol{p}$ is chosen from $\{\boldsymbol{p}, \boldsymbol{q}, \boldsymbol{r}\}$; that is, $\boldsymbol{p}$ is better than $\boldsymbol{q}$ at the ex-post stage. The conditions are related to shame. The decision maker prefers $\{\boldsymbol{p}\}$ to $\{\boldsymbol{p}, \boldsymbol{q}\}$. We call the doubleton $\{\boldsymbol{p}, \boldsymbol{q}\}$ shame-driven if the three conditions are satisfied.

Axiom 16. (Shame): For any $\boldsymbol{p}, \boldsymbol{q} \in \Delta^{I}$ such that $\{\boldsymbol{p}, \boldsymbol{q}\}$ is a shame-driven menu,

$$
\{\boldsymbol{p}\} \succeq\{\boldsymbol{p}, \boldsymbol{q}\}
$$

We rule out the effects on both pride and temptation.

Axiom 17. (Shame-Based Dominance): For any $A, B \in \mathcal{A}$, if (i) $A \sim_{S} B$ and (ii) $A \succeq B$, then $A \sim A \cup B$.

Proposition 7. Suppose that $\succeq$ satisfying Axiom 17 (Shame-based Dominance) is represented by a RDIC with a four-tuple $(u, \boldsymbol{\alpha}, \boldsymbol{\beta}, \boldsymbol{\gamma})$. $\succeq$ exhibits Shame (Axiom 16) if and only if $-1 \leq$ $\beta_{S}(\cdot) \leq 0$

\section{Comparative Statics}

We investigate the comparative attitude toward shame. Consider two decision makers, Mr. $X$ and Mr.Y, who acts as a decision maker 1. Assume that the set of other agents $S$ is fixed. Let $\succeq^{X}$ and $\succeq^{Y}$ be the two decision maker's preferences over menus.

Definition 8. $\succeq^{X}$ is more shame-averse than $\succeq^{Y}$ if the following holds; for any $\boldsymbol{p}, \boldsymbol{q} \in \Delta^{I}$ that is a shame-driven menu, and $l \in \Delta$,

$$
\{(l, \cdots, l)\} \succeq^{Y}\{\boldsymbol{p}, \boldsymbol{q}\} \Rightarrow\{(l, \cdots, l)\} \succeq^{X}\{\boldsymbol{p}, \boldsymbol{q}\} .
$$


Remember that firstly the doubleton $\{\boldsymbol{p}, \boldsymbol{q}\}$ is shame-driven, and $\boldsymbol{p}$ is better than $\boldsymbol{q}$ at the ex-post stage. The decision maker would feel shame, by choosing $\boldsymbol{p}$. Second, a singleton $\{(l, \cdots, l)\}$ does not cause any psychological effect including inequity aversion. Hence, the binary relation $\{(l, \cdots, l)\} \succeq\{\boldsymbol{p}, \boldsymbol{q}\}$ stems from shame.

Proposition 8. Suppose that $\succeq^{j}$ is represented by a RDIC with a four-tuple $\left(u, \boldsymbol{\alpha}, \boldsymbol{\beta}^{j}, \boldsymbol{\gamma}\right)$ where $j \in\{X, Y\}$. Then, $\succeq^{X}$ is more shame-averse than $\succeq^{Y}$ if and only if $-1 \leq \beta_{X}(\cdot)<\beta_{Y}(\cdot) \leq 0$.

\section{Shame-Aversion causes Shame: Guilt-Avoidance}

We study the case of ex-ante shame-aversion $\left(\gamma_{S}=1\right)$, which causes shame $\left(-1<\beta_{S}(\cdot) \leq 0\right)$. Even if the ex-ante choices are not publicly observed, the decision maker may feel shame. As mentioned in Introduction, the notion is similar to guilt.

We provide the following axiom.

Axiom 18. (Social-Interest): For any $A, B \in \mathcal{A}, A \succeq_{S} B \Rightarrow A \sim_{S} A \cup B$.

This axiom is reminiscent of Self-Interest (Axiom 13). The axiom says that, at the ex-ante stage, in terms of social interests, the decision maker predicts that adding $B$ into $A$ does not have any effect on resulting behavior at the ex-post stage.

Corollary 4. Suppose that $\succeq$ is represented by a RDIC with a four-tuple $(u, \boldsymbol{\alpha}, \boldsymbol{\beta}, \boldsymbol{\gamma})$. Then, $\succeq$ exhibits Social-Interest Dominance (Axiom 18) if and only if $\gamma_{S}=1$.

We relax Shame (Axiom 16) to capture a relationship between guilt-aversion and shame.

Axiom 19. (Consistency on Shame): For any $\boldsymbol{p}, \boldsymbol{q} \in \Delta^{I}$ such that $\{\boldsymbol{p}, \boldsymbol{q}\}$ is a shame-driven menu, if $\{\boldsymbol{p}\} \sim_{S}\{\boldsymbol{p}, \boldsymbol{q}\}$, then $\{\boldsymbol{p}\} \succeq\{\boldsymbol{p}, \boldsymbol{q}\}$.

Proposition 9. Suppose that $\succeq$ satisfying Shame-Based Dominance (Axiom 17) is represented by a RDIC with a four-tuple $(u, \boldsymbol{\alpha}, \boldsymbol{\beta}, \boldsymbol{\gamma})$ with $\beta_{1}\left(u(\Delta)^{2}\right)=0$. If $\succeq$ satisfy Social-interest Dominance (Axiom 18) and Consistency on Shame (Axiom 19), then $-\infty<\beta_{S}\left(u(\Delta)^{2}\right) \leq 0$ and $\gamma_{S}=1$.

Proposition 9 says that the additional axioms (Axioms 18, 17 and 19) characterize the attitude toward social image concerns $\left(\beta_{1}\left(u(\Delta)^{2}\right)=0\right.$ and $\left.-\infty<\beta_{S}\left(u(\Delta)^{2}\right) \leq 0\right)$. First, $\beta_{1}\left(u(\Delta)^{2}\right)=0$ implies that there is no effect on pride and temptation. Next, $\gamma_{S}=1$ implies that the decision maker chooses his reference point as others' maximal element. This can be interpreted as guilt aversion. Finally, $-\infty<\beta_{S}\left(u(\Delta)^{2}\right) \leq 0$ states that the decision maker feels shame at the ex-post stage, stemming from guilt avoidance (see Table 2).

\section{Reference Point Formation}

We study the effect on reference-point formation for prosocial behavior. 


\subsection{Comparative Statics on Reference Points}

We study comparative statics on reference points $(\gamma)$. Consider two decision makers $X$ and $Y$. Both Mr.X and Mr. $Y$ are denoted by a decision maker 1. Assume that the set of other passive agents $S$ is fixed. Let $\succeq_{j}^{h}(h \in\{X, Y\})$ be the decision maker $h$ 's induced menu preferences conditional on $j \in\{1, S\}$.

Definition 9. Suppose that $\succeq^{X}$ and $\succeq^{Y}$ satisfies the axioms in Theorem 1 . We say that $\succeq_{1}^{X}$ is more self-interested than $\succeq_{1}^{Y}$ if for any $A, B \in \mathcal{A}$ with $B \subset A$,

$$
A \succeq_{1}^{Y} B \Rightarrow A \succeq_{1}^{X} B
$$

The definition says that in terms of self-interested image-conscious preferences $\left(\succeq_{1}\right)$, if the decision maker $Y$ prefers larger menus, then the decision maker $X$ also does. Since $\succeq_{1}$ reflects $\succsim_{1}$, preferring larger menus is not worse off in terms of individual preferences (at the ex-ante stage).

Definition 10. Suppose that $\succeq^{X}$ and $\succeq^{Y}$ satisfies the axioms in Theorem 1. We say that $\succeq_{S}^{X}$ is more socially-interested than $\succeq_{S}^{Y}$ if for any $A, B \in \mathcal{A}$ with $B \subset A$,

$$
A \succeq{ }_{S}^{Y} B \Rightarrow A \succeq_{S}^{X} B
$$

In the same way, in terms of social-interested image-conscious preferences $\left(\succeq_{S}\right)$, if the decision maker $Y$ prefers larger menus, then the decision maker $X$ also does. Since $\succeq_{S}$ reflects $\succsim^{S}$, preferring larger menus is not worse off in terms of social preferences (at the ex-ante stage).

We obtain the following result.

Proposition 10. Suppose that for each $h \in\{X, Y\}, \succeq^{h}$ is represented by a four-tuple $\left(u, \boldsymbol{\alpha}, \boldsymbol{\beta}, \boldsymbol{\gamma}^{h}\right)$. Then, the following statements hold:

(i) $\succeq_{1}^{X}$ is more temptation-driven than $\succeq_{1}^{Y}$ if and only if $\gamma_{1}^{X} \geq \gamma_{1}^{Y}$.

(ii) $\succeq_{S}^{X}$ is more shame-averse than $\succeq_{S}^{Y}$ if and only if $\gamma_{S}^{X} \geq \gamma_{S}^{Y}$.

\subsection{Choice Set Effects}

We study choice set effects on social image concerns. Throughout of the analysis, fix the set of all agents, $I$.

Consider a doubleton $\{\boldsymbol{p}, \boldsymbol{q}\}$. We consider the two cases. The first one is the case that a selfish allocation $\boldsymbol{r}$ is added into the doubleton. The second one is the case that an altruistic allocation $\boldsymbol{r}^{\prime}$ is added into the doubleton. ${ }^{17}$

\footnotetext{
${ }^{17}$ The selfish allocation and the altruistic allocation are defined later.
} 


\section{Choice Set Effects with Pride and Temptation}

First, we study the case that a selfish allocation $\boldsymbol{r}$ is added.

Definition 11. We say that an allocation $\boldsymbol{r}$ is selfish for a menu $A$ if for all $\boldsymbol{p} \in A, r_{1} \succ^{1} p_{1}$.

The following definition is related to the attitude toward temptation and pride with choice-set effects.

Definition 12. Take allocations $\boldsymbol{p}, \boldsymbol{q} \in \Delta^{I}$ and, suppose that an allocation $\boldsymbol{r}$ is more selfish than $\boldsymbol{p}, \boldsymbol{q}$.

(i) Suppose that $\{\boldsymbol{p}, \boldsymbol{q}\}$ is a selfishly conflicting menu. $\succeq$ exhibits more temptation-driven preferences if $\{\boldsymbol{p}\} \succeq\{\boldsymbol{p}, \boldsymbol{q}\} \Rightarrow\{\boldsymbol{p}\} \succeq\{\boldsymbol{p}, \boldsymbol{r}\}$.

(ii) Suppose that $\{\boldsymbol{p}, \boldsymbol{q}\}$ is a socially conflicting menu. $\succeq$ exhibits more pride-seeking preferences if $\{\boldsymbol{p}, \boldsymbol{q}\} \succeq\{\boldsymbol{p}\} \Rightarrow\{\boldsymbol{p}, \boldsymbol{r}\} \succeq\{\boldsymbol{p}\}$.

The following result characterizes image-conscious behavior with the choice-set effect in the case of adding selfish allocations. Let us introduce some notation. For each menu $A \in \mathcal{A}$, denote $\overline{\boldsymbol{u}}_{A}:=\left(\bar{u}_{1}(A), \bar{u}_{S}(A)\right)$ where the first argument is the reference level of the decision maker's own allocations, and the second argument is the reference level of other passive agents' allocations.

Proposition 11. The following statements hold:

(i) $\succeq$ exhibits more temptation-driven preferences if and only if $\beta_{1}$ is increasing in the first argument on $(0,+\infty)$.

(ii) $\succeq$ exhibits more pride-seeking preferences if and only if $\beta_{1}$ is decreasing in the first argument on $(-1,0)$.

In Proposition 11, consider the first case of temptation-driven preferences. As a selfish allocation $\boldsymbol{r}$ is added into the menu $\{\boldsymbol{p}, \boldsymbol{q}\}$, the reference level of $\bar{u}_{1}$ increases. Notice that $\boldsymbol{p}$ is chosen from the menu $\{\boldsymbol{p}, \boldsymbol{q}\}$. Due to temptation-driven preferences, $\boldsymbol{q}$ can be chosen from the menu $\{\boldsymbol{p}, \boldsymbol{q}, \boldsymbol{r}\}$, since $\beta_{1}\left(\overline{\boldsymbol{u}}_{\{\boldsymbol{p}, \boldsymbol{q}\}}\right) \leq \beta_{1}\left(\overline{\boldsymbol{u}}_{\{\boldsymbol{p}, \boldsymbol{q}, \boldsymbol{r}\}}\right)$. Hence, a violation of WARP can occur due to temptation-driven preferences. In the same way, pride-seeking preferences may lead to another violation of WARP.

\section{Choice Set Effects with Shame}

We study the case that an altruistic allocation $\boldsymbol{r}^{\prime}$ is added. Fix a menu $\{\boldsymbol{p}, \boldsymbol{q}\}$.

Definition 13. We say that an allocation $\boldsymbol{r}^{\prime}$ is altruistic if for all $\boldsymbol{p} \in A, r_{S} \succ^{S} p_{S}$. 


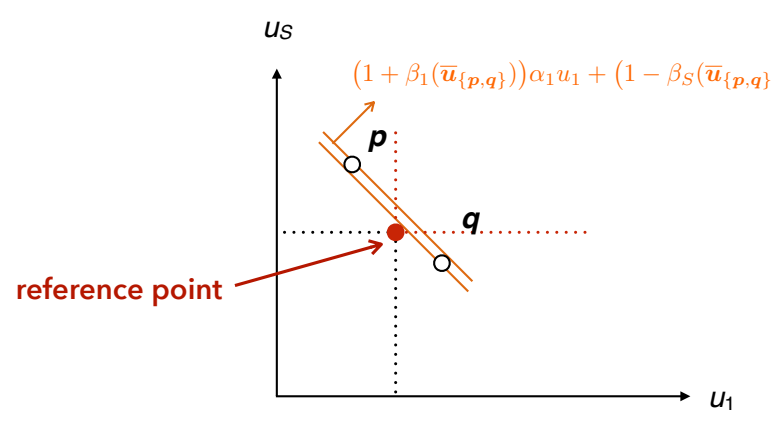

(a) Choice-Set $\{\boldsymbol{p}, \boldsymbol{q}\}$

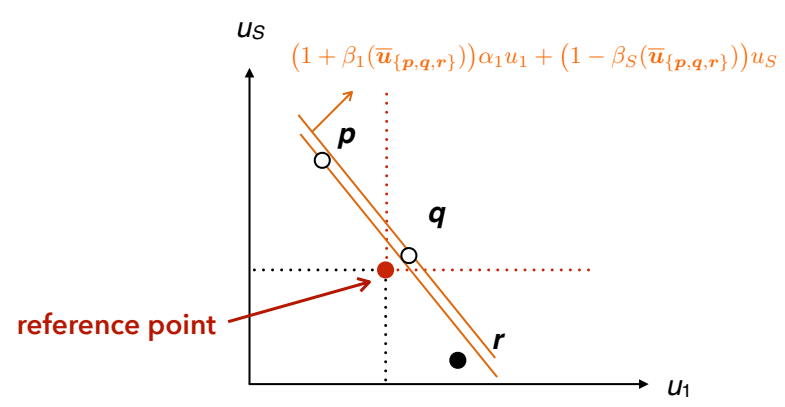

(b) Choice-Set $\{\boldsymbol{p}, \boldsymbol{q}, \boldsymbol{r}\}$

Figure 3: Choice-Set Effect and Temptation to Act Selfishly

The following definition is related to the attitude toward shame with choice-set effects.

Definition 14. Consider a menu $\{\boldsymbol{p}, \boldsymbol{q}\}$ that is shame-driven and an allocation $\boldsymbol{r}^{\prime}$ that is more altruistic than $\boldsymbol{p}, \boldsymbol{q}$. We say that $\succeq$ exhibits more shame-averse preferences if

$$
\{\boldsymbol{p}\} \succeq\{\boldsymbol{p}, \boldsymbol{q}\} \Rightarrow\{\boldsymbol{p}\} \succeq\left\{\boldsymbol{p}, \boldsymbol{r}^{\prime}\right\}
$$

The following result characterizes a prosocial behavior with the choice-set effect in the case of adding altruistic allocations.

Proposition 12. Consider a menu $\{\boldsymbol{p}, \boldsymbol{q}\}$ that is shame-driven and an allocation $\boldsymbol{r}^{\prime}$ that is more altruistic than $\boldsymbol{p}, \boldsymbol{q}$. Then, the following statements hold: $\succeq$ exhibits more shame-averse preferences if and only if $\beta_{S}$ is decreasing in the second arguments on $(-\infty, 0)$.

As an altruistic allocation $\boldsymbol{r}^{\prime}$ for $\{\boldsymbol{p}, \boldsymbol{q}\}$ is added into the menu $\{\boldsymbol{p}, \boldsymbol{q}\}$, the reference level of $\bar{u}_{S}$ increases. Notice that $\boldsymbol{p}$ is chosen from the menu $\{\boldsymbol{p}, \boldsymbol{q}\}$. Due to shame-averse preferences, $\boldsymbol{q}$ can be chosen from the menu $\left\{\boldsymbol{p}, \boldsymbol{q}, \boldsymbol{r}^{\prime}\right\}$, as $\beta_{S}\left(\overline{\boldsymbol{u}}_{\{\boldsymbol{p}, \boldsymbol{q}\}}\right) \leq \beta_{S}\left(\overline{\boldsymbol{u}}_{\{\boldsymbol{p}, \boldsymbol{q}, \boldsymbol{r}\}}\right)$ because of the decreasing function of $\beta_{S}$ with respect to the second argument. Thus, a violation of WARP can occur due to shame-averse preferences.

\section{A Relationship between Social Image and Reciprocity}

By applying social image into strategic environments, we study a relationship between social image and intrinsic reciprocity. The theory of reciprocity refers to a kindness to act kindly in response to kind actions, which is called positive reciprocity (Sobel (2005)). ${ }^{18}$ If the decision

\footnotetext{
${ }^{18}$ Both positive and negative reciprocity can be interpreted as conforming to social norm.
} 
maker predicts that his opponent chooses an altruistic action, then he responds to the opponent's altruistic action, and he also tries to choose an altruistic action. On the other hand, negative reciprocity is similarly captured in the same way. If the decision maker predicts that her opponent chooses a selfish action, then he prepares acting selfishly.

Prisoners' Dilemma. Consider a one-shot prisoner's dilemma game in Table 3 . Let $g, l>0$ with $1>g-l$.

Table 3: A Prisoner's Dilemma Game

\begin{tabular}{|c|c|c|}
\hline Prisoner 1/ Prisoner 2 & Cooperation & Defection \\
\hline Cooperation & 1,1 & $-l, 1+g$ \\
\hline Defection & $1+g,-l$ & 0,0 \\
\hline
\end{tabular}

Reciprocal behavior can be also captured by social image concerns, if the decision maker cares about how his behavior is perceived by opponents. If an opponent tries to act altruistically, then he may feel shame. Then, social image concern can lead to altruistic behavior. However, social image concerns can capture broader behavioral patterns. When an opponent tries to choose $C$ (cooperation) in a (one-shot) prisoners' dilemma (PD) game, the decision maker feels much temptation to act selfishly, which leads to choose $D$ (defection). This is not consistent with the concept of intrinsic reciprocity.

We study a relationship between intrinsic reciprocity and social image concerns. In particular, we study a condition in which cooperation can be sustained in the PD game, and explicitly study the role of social image. First, we verify that there exists a Nash equilibrium under the RDIC players. Second, we study the case that a cooperation is sustained with probability 1 .

\subsection{Nash Equilibrium}

Notation. We set up a simple model. For simplicity, suppose that players, denoted by $i \in I=\{1, \cdots, n\}$, have the same space of outcomes, denoted by $X$. We denote the space of simple lotteries over $X$ by $\Delta(X)$. For simplicity, let us write it down $\Delta:=\Delta(X)$. Let $\boldsymbol{s}_{i}=\left\{s_{i}^{1}, \cdots, s_{i}^{n_{i}}\right\}$ be the finite set of pure strategies for each player $i \in I$. Let the outcome function be denoted by $\mathcal{O}: \prod_{i=1}^{n} s_{i} \rightarrow \prod_{i=1}^{n} X_{i}$. Let $\Sigma_{i}$ be the space of mixed strategies of player $i$. In the same way, let the allocation function be denoted by $\mathcal{O}_{\Delta}: \prod_{i=1}^{n} \Sigma_{i} \rightarrow$ $\prod_{i=1}^{n} \Delta\left(X_{i}\right)$. Let $\Sigma:=\prod_{i=1}^{n} \Sigma_{i}$. Let $\mathcal{A}$ be the set of all non-empty compact subsets of $\Sigma$.

Let $\mathcal{G}=\left\langle I,\left(A_{i}\right)_{i \in I},\left(V_{i}\right)_{i \in I}\right\rangle$ be a game. Given a game $\mathcal{G}$, suppose that each player $i$ has a preference relation over $\mathcal{A}$, and faces a normal-form game $\mathcal{G}$ with $A_{i}$. $V_{i}$ is a utility of menus. 
For each player $i \in I, \sigma_{-i}$ is represented by player $i$ 's belief about how $i$ 's opponents play the game.

We assume explicitly the following. Fix a game $\mathcal{G}$. For each player $i \in I$, player $i$ thinks that player $i$ evaluates player $j$ 's action on the basis of what player $j$ thinks that $i$ is going to do $\left(\sigma^{*}\right)$, and that how the player $i$ 's action is perceived by opponents.

We study a binary relation $\succeq$ on $\mathcal{A}$, i.e., preferences over sets of mixed strategies. In the following, we study a conditional preference on mixed strategies, i.e., $\succeq_{i, \sigma^{*}}$. Hence, we explicitly study a context-dependent preference.

Utility. The utility representation in Theorem 1 is a utility of menus. Since we consider a fixed game, the opportunity set (menu) is, in advance, determined for each player. As mentioned above, their menu-preferences are conditional on the mixed strategies of other players $\left(\sigma_{-i}\right)$. Since the game $\mathcal{G}$ is fixed, given a mixed-strategy profile of other players, the ex-post utility of player $i$ is given in the following way.

$$
U_{i}\left(\sigma_{i}^{*} \mid \sigma_{-i}\right)=\left(1+\beta_{i}\left(\overline{\boldsymbol{u}}_{\mathcal{G}}\right)\right) \alpha_{i} u_{i}\left(\sigma_{i}^{*}\right)+\left(1-\beta_{-i}\left(\overline{\boldsymbol{u}}_{\mathcal{G}}\right)\right) \sum_{j \neq i} \alpha_{j} u_{i}\left(\sigma_{j}^{*}\right)
$$

where $\overline{\boldsymbol{u}}_{\mathcal{G}}$ is the reference point of the game $\mathcal{G}$.

The ex-post utility is a weighted utilitarian, as mentioned in Theorem 1.

Nash Equilibrium. We define a Nash equilibrium, in terms of the framework of this paper. The definition says that players choose a best action in the best-response way.

Definition 15. A Nash equilibrium is a strategy profile $\sigma^{*}$ in which player $i$ 's strategy $\sigma_{i}^{*}$ is maximal, according to $\succeq_{i, \sigma^{*}}$.

We obtain the following corollary of the existence of the Nash equilibrium. The corollary guarantees that if $\succeq_{i}$ satisfies the axioms in Section 2, then a Nash equilibrium exists for the fixed game $\mathcal{G}$.

Corollary 5. Given a game $\mathcal{G}$, if each $\succeq_{i}$ satisfies the axioms in Theorem 1, then there exists a Nash equilibrium.

Example 1. (Nash Equilibrium in Table 3): We provide an example of prisoners' dilemma (PD) game with social image concerns. Assume that Prisoner 1 takes $C$ with probability $p$, and takes $D$ with probability $1-p$. Then, a mixed strategy Nash equilibrium is as follows: ${ }^{19}$

$$
p^{*}=\frac{\left(1+\beta_{1}\left(\overline{\boldsymbol{u}}_{\mathcal{G}}\right)\right) l-\left(1-\beta_{2}\left(\overline{\boldsymbol{u}}_{\mathcal{G}}\right)\right)(1+g)}{\left(1+\beta_{1}\left(\overline{\boldsymbol{u}}_{\mathcal{G}}\right)\right)(g+l)-\left(1-\beta_{2}\left(\overline{\boldsymbol{u}}_{\mathcal{G}}\right)\right)(g-l)} .
$$

\footnotetext{
${ }^{19}$ In the same way, Prisoner 2' s mixed strategy is obtained.
} 


\subsection{Intrinsic Reciprocity}

We study a relationship between intrinsic reciprocity and social image concerns. In particular, we focus on the study in the case of one-shot Prisoners' Dilemma (PD) games (in Table 3).

By arranging the terms, we have the following ex-post RDIC representation.

$$
U_{i}^{*}\left(\sigma^{*}\right)=u_{i}\left(\sigma_{i}^{*}\right)+\frac{1-\beta_{j}\left(\overline{\boldsymbol{u}}_{\mathcal{G}}\right)}{1+\beta_{i}\left(\overline{\boldsymbol{u}}_{\mathcal{G}}\right)} u_{i}\left(\sigma_{j}^{*}\right)
$$

where $i \neq j$. By Theorem 1, the parameter $\frac{1-\beta_{j}\left(\overline{\boldsymbol{u}}_{\mathcal{G}}\right)}{1+\beta_{i}\left(\overline{\boldsymbol{u}}_{\mathcal{G}}\right)}$ is uniquely identified (see Proposition 1). The ex-post utility can be interpreted as a special case of the Rabin-type reciprocial utility model (see Sobel (2005), Segal and Sobel (2007) etc.).

Segal and Sobel (2007) provides an axiomatic foundation for preferences for reciprocity, which represents a weighted utilitarian. The weight a decision maker places on an opponent's action depends on the players' joint behavior. In the following, to compare this model with Segal and Sobel (2007), assume that $\alpha_{i}=1$, i.e., there is no effect on pure altruism. ${ }^{20}$

\section{Positive Reciprocity}

Fix $i \in I$ where $I=\{1,2\}$. We focus on the behaviors of player $i$. Formally, we write down the parameter on social image of player $i$ by $\beta_{i}^{i}$ and $\beta_{S}^{i}$, but in the following, we drop $i$.

First, we study the effect on shame of acting selfishly. The following result corresponds to the case of positive reciprocity, in that the strategy profile $(C, C)$ can be a Nash Equilibrium, if the statement holds for all players $i \in I$.

Example 2. Given a game $\mathcal{G}$ with $|I|=\{1,2\}$, suppose that the opponent of player $i$ takes $C$. Then, player $i$ takes $C$ if

$$
\beta_{j}\left(\overline{\boldsymbol{u}}_{\mathcal{G}}\right) \leq 1-\left(1+\beta_{i}\left(\overline{\boldsymbol{u}}_{\mathcal{G}}\right)\right) \frac{g}{1+l} .
$$

Example 2 says that when the opponent chooses $C$, i.e., $\sigma_{2}^{*}=\delta_{C}$, player $i$ also chooses $C$ if the condition is satisfied.

Second, we study the effect on temptation to act selfishly. In this case, the strategy profile $(C, C)$ may not be a Nash Equilibrium. Suppose that player $i$ predicts that the opponent $j$ takes $C$. Then, if player $i$ feels temptation to act selfishly, and temptation beats shame, i.e., $\beta_{j}>\left(1+\beta_{i}\right) \frac{g}{1+l}$, then player $i$ chooses $D$.

\section{Negative Reciprocity}

In the similar way, we study the case of negative reciprocity.

\footnotetext{
${ }^{20}$ Even though we do not study a relationship between pure altruism and reciprocity, the study is helpful for a deeper understanding of generous reciprocity.
} 
Example 3. Given a game $\mathcal{G}$ with $|I|=2$, suppose that the opponent of player $i$ takes $D$. Then, player $i$ takes $D$ if

$$
\beta_{j}\left(\overline{\boldsymbol{u}}_{\mathcal{G}}\right) \geq 1-\left(1+\beta_{i}\left(\overline{\boldsymbol{u}}_{\mathcal{G}}\right)\right) \frac{l}{1+g} .
$$

In Example 3, we do not require that the opponent of player $i$ exhibits temptation to act selfishly. If $\beta_{j}>\left(1-\beta_{i}\right) \frac{l}{1+g}$, player $i$ feels a shame of reacting to a negative reciprocity.

\section{Negative Reciprocity with Anger}

Emotions can change objective payoffs in a way that increases fitness. Consider the intersting story introduced in Gilboa (2009). ${ }^{21}$ In the story, an evolutionary foundation for utility is discussed. Here, we provide an example of foundations of utility stemming from social emotions.

There are two players: a tiger and a she-bear. The she-bear tries to get food for her cubs, whom she leaves behind. The tiger finds the cobs due to their smells, and needs to determine whether to "attack" the cubs or not. The strategic environment is described by an extensive-form game (see Figure 4). If the tiger passes, then nothing happens. Both get the payoff 0 . If the tiger attacks the cubs, then the bear has two options. One is that the she-bear retaliates against the tiger. Then, both the she-bear and the tiger will be hurt. As a result, both get the payoff -20 . If the she-bear goes on without fighting, then the tiger kills the cubs, and get the payoff 10. The tiger will not be hurt, so she gets the payoff 0 .

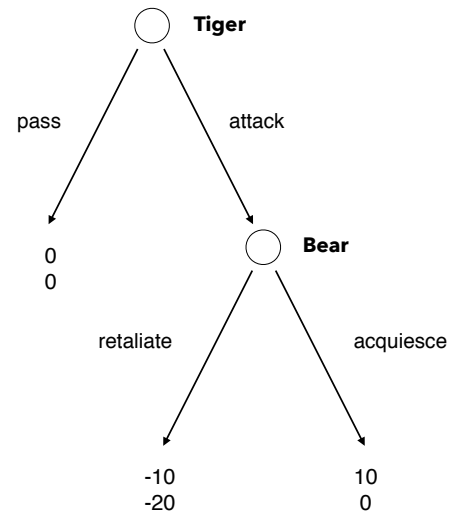

(a) Extensive-Form Game without Social Emotion

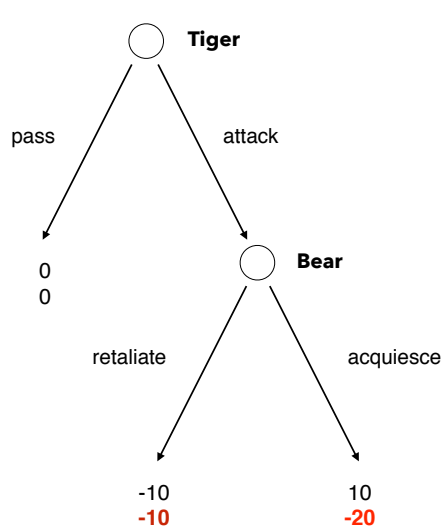

(b) Extensive-Form Game with Social Emotion

Figure 4: Reciprocity and Social Emotion

\footnotetext{
${ }^{21}$ See Chapter 9; pages 139 - 142.
} 
In the extensive-form game, there might be obviously two pure strategy Nash equilibria (Gilboa (2009)). In the first Nash equilibrium, the tiger attacks and the she-bear walks away. In the second Nash equilibrium, the tiger passes, given that the she-bear retaliate in case her cubs are eaten. Notice that, of course, the latter one is not sub-game perfect. The she-bear's action can be just a non-credible threat.

\section{The Role of Pride}

The she-bear may care not only about herself, but also about her cubs. When the she-bear retaliates against the tiger, she gets the payoff -20 as mentioned above. We interpret the payoff as her physical damage; that is, the she-bear gets the payoff -20 , and her cub gets nothing. On the other hand, when the she-bear walks away (acquiesces), she has no hurt, so she gets the payoff 0 . However, her cubs are eaten, so her cubs get the payoff $-10 .^{22}$ If the she-bear exhibits social emotions such as pride and shame, then the action of fighting can become a credible threat. Such a subjective payoff for the she-bear may change in Figure ??.

We consider the ex-post utility of the RDIC. For simplicity, there is one her cub. Let the she-bear denote by 1 , and her cub 2. Assume that $\alpha_{1}=1$. Then, we only consider the impacts on social image concerns. Let the extensive-form game denote by $\mathcal{G}$. The factors on social image are as follows: $\beta_{1}\left(\overline{\boldsymbol{u}}_{\mathcal{G}}\right)=-0.5$ and $\beta_{2}\left(\overline{\boldsymbol{u}}_{\mathcal{G}}\right)=-1$. The ex-post utilities are as follows:

$$
\begin{aligned}
U(\text { retaliate }) & =-10=(1-0.5) \cdot(-20)+(1-(-1)) \cdot 0 \\
& >-20=(1-0.5) \cdot 0+(1-(-1)) \cdot(-10)=U(\text { acquiesce }) .
\end{aligned}
$$

Social emotions such as pride and shame can make the she-bear's credible threat. The tiger may feel anger of the she-bear, and then tend not to attack her cubs. This evolutionary viewpoint is closely related to social image concerns, because the she-bear deliberately cases about how her behavior is perceived by the tiger. Notice that the utility of the tiger is not included into the utility of the she-bear.

\section{Literature Review}

In this section, we provide the literature review. We mainly discuss the three related studies: (i) Dillenberger and Sadowski (2012), (ii) Evren and Minardi (2017), and (iii) Saito (2015). These studies take preferences over menus as primitives. The studies axiomatically identify the factors behind social image concerns.

\footnotetext{
${ }^{22}$ We assume that this payoffs for her cubs are estimated by the she-bear. Here, we assume that the damage through the fight against the tiger is more worse than it.
} 


\section{Dillenberger and Sadowski (2012): Shame of Acting Selfishly}

Dillenberger and Sadowski (2012) develop the theory of shame of acting selfishly. ${ }^{23}$ The shame is captured by a personal norm function $\varphi$ and a function $g$. Because of shame, the decision maker may deviate from WARP. On the other hand, in this paper, shame is captured by the pair $\left(\beta_{S}(\cdot), \gamma_{S}\right)$. The factor of shame is determined by choice sets, and the level of shame is changeable based on choice sets.

To compare this paper with Dillenberger and Sadowski (2012), we provide a weaker version of Axiom 7 (Weak Independence).

Axiom 20. (Shame-Based Independence): For any $A, B, C \in \mathcal{A}$ and $\lambda \in[0,1]$,

$$
A \succ_{S} B \Rightarrow \lambda A+(1-\lambda) C \succ \lambda B+(1-\lambda) C .
$$

Corollary 6. $\succeq$ satisfies Standard Preferences (Axiom 1), Consistency (Axiom 2), Pareto (Axiom 3), Singleton Independence (Axiom 6), Social Interest (Axiom 18), Shame-Based Dominance (Axiom 17), and Shame-Based Independence (Axiom 20) if and only if there exists a four-tuple $(u, \boldsymbol{\alpha}, \boldsymbol{\beta}, \boldsymbol{\gamma})$ with $\beta_{1}(u(\Delta))=0$, such that $\succeq$ is represented by

$$
V(A)=\max _{\boldsymbol{p} \in A}\left[\sum_{i \in I} \alpha_{i} u\left(p_{i}\right)-\beta_{S}\left(\max _{\boldsymbol{q} \in A} \sum_{i \in S} \alpha_{i} u\left(q_{i}\right)\right)\left(\sum_{i \in S} \alpha_{i} u\left(p_{i}\right)-\max _{\boldsymbol{q} \in A} \sum_{i \in S} \alpha_{i} u\left(q_{i}\right)\right)\right] .
$$

where $\beta_{S}: u(\Delta) \rightarrow(-\infty, 1)$

\section{Evren and Minardi (2017): Warm-Glow and Freedom to be Selfish}

Evren and Minardi (2017) develop a theory of "warm glow," which refers to other-serving behavior that is valuable for the decision maker, apart from its social implications. ${ }^{24}$ They apply the theory of warm glow to an individual who enjoys taking prosocial behavior that incurs a private cost. Such a prosocial decision-making improves his social image. The decision maker behaves prosocially by viewing "warm glow" as a form of preferences for larger choice sets driven by the desire to have the freedom to be selfish. On the other hand, in this paper, a different notion of "warm glow" is captured by the pair $\left(\beta_{S}(\cdot), \gamma_{S}\right)$.

To compare this paper with Evren and Minardi (2017), we provide a weaker version of Axiom 7 (Weak Independence).

Axiom 21. (Pride-Based Independence): For any $A, B, C \in \mathcal{A}$ and $\lambda \in[0,1]$,

$$
A \succ_{1} B \Rightarrow \lambda A+(1-\lambda) C \succ \lambda B+(1-\lambda) C .
$$

\footnotetext{
${ }^{23}$ Neilson (2009) also presents a context-dependent model to study the effect on kindness, reluctance, and shame.

${ }^{24}$ See also Andreoni (1989, 1990), which are seminal studies on warm glow. Specifically, the Evren and Minardi (2017)'s model is based on the notion of warm glow to the freedom to be selfish.
} 
Corollary 7. $\succeq$ satisfies Standard Preferences (Axiom 1), Consistency (Axiom 2), Pareto (Axiom 3), Singleton Independence (Axiom 6), Self-Interest (Axiom 13), Pride-Based Dominance (Axiom 12), and Pride-Based Independence (21) if and only if there exists a four-tuple $(u, \boldsymbol{\alpha}, \boldsymbol{\beta}, \boldsymbol{\gamma})$ with $\beta_{S}(u(\Delta))=0$, such that $\succeq$ is represented by

$$
V(A)=\max _{\boldsymbol{p} \in A}\left[\sum_{i \in I} \alpha_{i} u\left(p_{i}\right)+\beta_{1}\left(\max _{\boldsymbol{q} \in A} u\left(q_{1}\right)\right)\left(\alpha_{1}\left(u\left(p_{1}\right)-\max _{\boldsymbol{q} \in A} u\left(q_{1}\right)\right)\right)\right] .
$$

where $\beta_{1}: u(\Delta) \rightarrow(-1,+\infty)$.

\section{Saito (2015): Image-Conscious Utilitarian}

Saito (2015) develops a rational choice theory which uniquely identifies three emotional factors behind social image: (i) pride in acting altruistically, (ii) shame of acting selfishly, and (iii) temptation to act selfishly. His generalized utilitarian model satisfies WARP in ex-post choices unlike this paper (see Proposition 5 (p.354) in Saito (2015)).

To compare this paper with Saito (2015), we consider the case that the reference points are the maximal values on both self-allocations and others' allocations, i.e., $\gamma_{1}=\gamma_{S}=1$.

Corollary 8. $\succeq$ satisfies Standard Preferences (Axiom 1), Consistency (Axiom 2), Pareto (Axiom 3), Singleton Independence (Axiom 6), Weak Independence (Axiom 7), Self-interest (Axiom 13), and Social Interest (Axiom 18) if and only if there exists a four-tuple $(u, \boldsymbol{\alpha}, \boldsymbol{\beta}, \boldsymbol{\gamma})$ such that $\succeq$ is represented by

$$
\begin{aligned}
V(A)=\max _{\boldsymbol{p} \in A}\left[\sum_{i \in I} \alpha_{i} u\left(p_{i}\right)\right. & +\beta_{1}\left(\max _{\boldsymbol{q} \in A} u\left(q_{1}\right)\right)\left(\alpha_{1}\left(u\left(p_{1}\right)-\max _{\boldsymbol{q} \in A} u\left(q_{1}\right)\right)\right) \\
& \left.-\beta_{S}\left(\max _{\boldsymbol{q} \in A} \sum_{i \in S} \alpha_{i} u\left(q_{i}\right)\right)\left(\sum_{i \in S} \alpha_{i} u\left(p_{i}\right)-\max _{\boldsymbol{q} \in A} \sum_{i \in S} \alpha_{i} u\left(q_{i}\right)\right)\right],
\end{aligned}
$$

where $\beta_{1}: u(\Delta) \rightarrow(-1,+\infty)$ and $\beta_{S}: u(\Delta) \rightarrow(-\infty, 1)$.

The case that $\boldsymbol{\beta}=\left(\beta_{1}, \beta_{S}\right)$ are constant corresponds to the general utilitarian in Saito (2015). We consider the following axiom on Independence.

Axiom 22. (Constant Effect on Social Emotions): For any $A, B \in \mathcal{A}, \boldsymbol{p}, \boldsymbol{q} \in \Delta^{I}$, and $\lambda \in[0,1]$, if $A \sim\{\boldsymbol{p}\}$ and $B \sim\{\boldsymbol{q}\}$,

$$
\lambda\{\boldsymbol{p}\}+(1-\lambda)\{\boldsymbol{q}\} \sim \lambda A+(1-\lambda) B .
$$

Axiom 22 is introduced in Mihm and Ozbek (2018), but we do not study self-regulation explicitly. ${ }^{25}$ Our model satisfies the following weaker version of Axiom 22.

Axiom 23. (Weak Constant Effect on Social Emotions): For any $A, B \in \mathcal{A}, \boldsymbol{p}, \boldsymbol{q} \in \Delta^{I}$, and $\lambda \in[0,1]$, if $A \sim_{j}\{\boldsymbol{p}\}$ and $B \sim_{j}\{\boldsymbol{q}\}$ for each $j \in\{1, S\}$,

$$
\lambda\{\boldsymbol{p}\}+(1-\lambda)\{\boldsymbol{q}\} \sim \lambda A+(1-\lambda) B .
$$

\footnotetext{
${ }^{25}$ Mihm and Ozbek (2018) call this axiom Neutral Desire for Commitment. This paper correspond to the case that the cost of self-regulation is constant.
} 


\section{Other Studies on Social Image Concerns}

We mention that there are some related studies on social image concerns. Yagasaki (2018) presents an axiomatic model of social comparisons. He modifies the axioms in Gul and Pesendorfer (2001) by explicitly introducing reference behavior of others as an additional primitive. In his model, the comparison with the most normative choices of others produce the feeling of pride or shame, which leads to prosocial behavior; that is, the decision maker feels pride or shame through the social comparison with others' actions. ${ }^{26}$

Bénabou and Tirole (2006) present a model of prosocial behavior, especially related to self-image concerns. In their model, prosocial behavior can occur stemming from an intrinsic motivation that is different from an extrinsic motivation (monetary incentive). The decision maker is image-conscious in the sense that he cares about how prosocial behavior contributes to the improvement of his image.

\section{Other Studies on Reference-Dependence}

Finally, we refer to some reference-dependent models, in particular, the studies on endogenous reference-point formations and range-based reasoning with the effect on choice sets. Köszegi and Rabin (2006) present a model of reference-dependence in which the decision maker endogenously forms a reference point with a belief on future returns. The concept of reference-point formation is different from ours, since in this paper, the decision maker determines reference points by using $\boldsymbol{\gamma}$. Ok et al. (2015) present a reference-dependent model by using revealed preference theory. In their study, a feasible alternative of choice sets can be a reference point. On the other hand, this paper allows for reference points that are not feasible. Kőszegi and Szeidl (2012) and Bushong et al. (2017) present a model of multiattribute utility representation, in which the weights on attributes depends on choice-sets, especially, range-dependence in attributes. This paper studies social image by depending on range-based reference points.

\section{Summary}

We close this section by providing a summary of the axiomatic studies on social image concerns in the following figure (Figure 5).

\footnotetext{
${ }^{26}$ In my own view, I may compare this paper with Yagasaki (2018) in the following way, even though the set-up of the model is different. The utility of menus is defined as follows:

$$
V(A)=\max _{\boldsymbol{p} \in A}\left[\alpha_{1} u\left(p_{1}\right)+\beta_{1}\left(\bar{u}_{S}(A)\right)\left(\alpha_{1} u\left(p_{1}\right)-\bar{u}_{S}(A)\right)\right],
$$

where $\bar{u}_{S}(A)$ can be interpreted as a belief on others's choices. The factor on pride or temptation depends on others' allocations. This representation is more general than that of Yagasaki (2018) in the sense that the factor on social image depends on reference points, and that the temptation to act selfishly $\left(\beta_{1}(\cdot) \in(-1,0)\right)$ is also captured.
} 


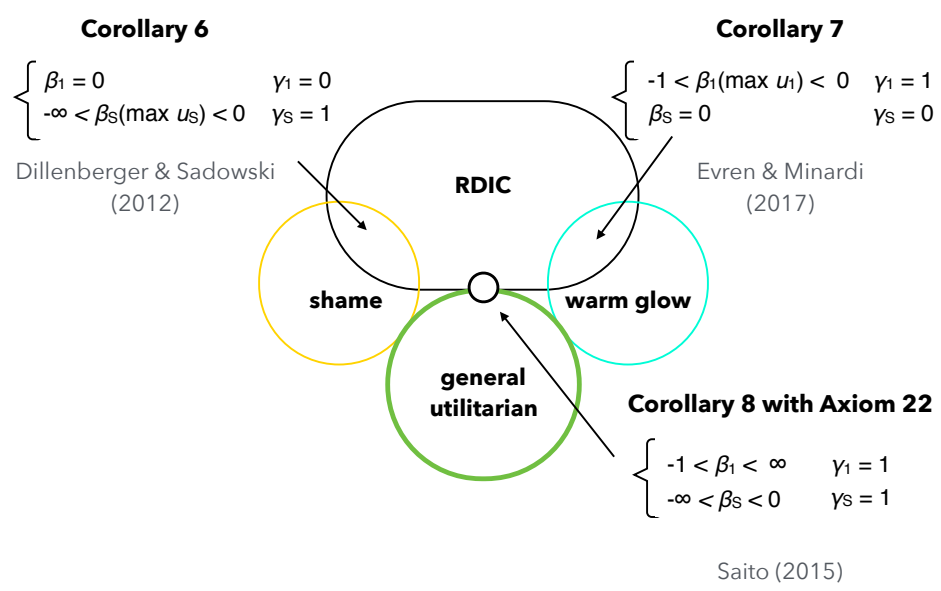

Figure 5: Related Studies on Social Image Concerns

\section{Concluding Remarks}

We have developed an axiomatic context-dependent model of social image concerns. Reference points can be a criterion for decision-making in social contexts. The model captures prosocial behavior arising from reference-dependent preferences. The building blocks of the model have a distinction between pure altruism and prosocial behavior. The pure altruism is captured by $\boldsymbol{\alpha}=\left(\alpha_{1},\left(\alpha_{i}\right)_{i \in S}\right)$. On the other hand, opportunity-dependent prosocial behavior is captured by a pair $(\boldsymbol{\beta}, \boldsymbol{\gamma})$. We have also studied a relationship between social image concern $(\boldsymbol{\beta})$ and reference-point formation $(\boldsymbol{\gamma})$.

The objective of this paper is to provide a behavioral foundation for social image concerns, in which the factors behind social image depend on reference points. Reference points are formed by caring about conflicts between selfishness and altruism in decision-making processes.

We have not considered any assumptions for a specific correlation between them. ${ }^{27}$ For example, some people may think that if a decision maker has the attitude toward pure altruism is relatively high, i.e., $\alpha_{1}$ is low, then her attitude toward prosocial behaviors is also high, i.e., $\gamma_{S}$ is high. Such a correlation should be studied in empirical/experimental studies as a future task.

There are some further tasks. As mentioned above, it is important to consider empirical/experimental studies on social image concerns. In an axiomatic viewpoint, the charac-

\footnotetext{
${ }^{27}$ I am grateful to Seiichi Fukui for his comment on correlation between social emotions.
} 
terization of the ex-post choices is a remaining task. Noor and Takeoka (2015) provides a characterization of menu-dependent self-control by using a pair $\langle\succeq, C\rangle$ where $C$ is a choice correspondence. In another theoretical viewpoint, social image concerns are also applied in strategic contexts. As mentioned in Introduction, social image concerns may depend on other players' type/personalities, other players' past actions, etc. This is also a future task. 


\section{A Proof of Theorem 1}

\section{A.1 Sufficiency Part}

We show the sufficiency part. Suppose that $\succeq$ satisfies the axioms in the main theorem (Theorem 1).

\section{Step 1}

In Step 1, first, we show that the two induced binary relations $\succsim^{j}$ on $\Delta^{I}(j \in\{1, S\})$ are well-defined. Next, we show that $\succsim^{j}$ on $\Delta^{I}(j \in\{1, S\})$ satisfies the axiom of Independence in the vNM-type expected utility theorem. Finally, we represent the first term of the utility representation by showing that $\alpha_{1}$ is positive.

$\succsim^{1}$ and $\succsim^{S}$ are well-defined. Remember that, for each $j \in\{1, S\}$, we define $\succsim^{j}$ on $\Delta^{I}$ as follows. The asymmetric and symmetric part of $\succsim^{j}$ are described by $\succ^{j}$ and $\sim^{j}$, respectively. For all $p_{1}, q_{1} \in \Delta, p_{1} \succsim_{1} q_{1}$ if $\left\{\left(p_{1}, r_{S}\right)\right\} \succeq\left\{\left(q_{1}, r_{S}\right)\right\}$ for some $r_{S} \in \Delta^{S}$. For all $p_{S}, q_{S} \in \Delta^{S}$, $p_{S} \succsim_{S} q_{S}$ if $\left\{\left(r_{1}, p_{S}\right)\right\} \succeq\left\{\left(r_{1}, q_{S}\right)\right\}$ for some $r_{1} \in \Delta$.

First, we show that $\succsim^{1}$ is well-defined. First, consider $\succsim^{1}$. We need to show that $p_{1} \succsim^{1} q_{1}$ if $\left(p_{1}, r_{S}\right) \succsim^{1}\left(q_{1}, r_{S}\right)$ for any $r_{S} \in \Delta^{S}$. Notice that for any $p_{j}, q_{j} \in \Delta^{j}$ and $\lambda \in[0,1]$, $\lambda p_{j}+(1-\lambda) q_{j} \in \Delta^{j}(j \in\{1, S\})$.

Suppose $\left\{\left(p_{1}, r_{S}\right)\right\} \succeq\left\{\left(q_{1}, r_{S}\right)\right\}$ and $\left\{\left(p_{1}, l_{S}\right)\right\} \prec\left\{\left(q_{1}, l_{S}\right)\right\}$. Consider $\left(p_{1}, l_{S}\right) \in \Delta^{I}$ and $\left(p_{1}, r_{S}\right) \in \Delta^{I}$. By Axiom 6 (Singleton Independence), letting $\lambda=\frac{1}{2},\left\{\left(p_{1}, \frac{1}{2} r_{S}+\frac{1}{2} l_{S}\right)\right\} \succeq$ $\left\{\left(\frac{1}{2} p_{1}+\frac{1}{2} q_{1}, \frac{1}{2} r_{S}+\frac{1}{2} l_{S}\right)\right\}$ and $\left\{\left(p_{1}, \frac{1}{2} r_{S}+\frac{1}{2} l_{S}\right)\right\} \prec\left\{\left(\frac{1}{2} p_{1}+\frac{1}{2} q_{1}, \frac{1}{2} r_{S}+\frac{1}{2} l_{S}\right)\right\}$. This is a contradiction. Hence, if there exists $r_{S} \in \Delta^{S}$ such that $\left\{\left(p_{1}, r_{S}\right)\right\} \succeq\left\{\left(q_{1}, r_{S}\right)\right\}$, then for any $r_{S} \in \Delta^{S},\left\{\left(p_{1}, r_{S}\right)\right\} \succeq\left\{\left(q_{1}, r_{S}\right)\right\}$. In the same way, we can show that $\succsim^{S}$ is well-defined. We omit it.

$\succsim^{1}$ and $\succsim^{S}$ satisfy Independence. Next, we show that for each $j \in\{1, S\}, \succsim^{j}$ satisfies the axiom of Independence in the expected utility theorem (EUT). Consider $\succsim_{1}$. By Axiom 1 (Standard Preferences), it is easily shown that $\succsim^{1}$ satisfies Completeness, Transitivity, and Mixture Continuity. We show that $\succsim^{1}$ satisfies the axiom of Independence: For any $p_{1}, q_{1}, r_{1}$ and $\lambda \in[0,1]$,

$$
p_{1} \succsim^{1} q_{1} \Leftrightarrow \lambda p_{1}+(1-\lambda) r_{1} \succsim^{1} \lambda q_{1}+(1-\lambda) r_{1} .
$$

Fix $p_{1}, q_{1}, r_{1} \in \Delta$ and $\lambda \in[0,1]$. Then, for any $p_{S}, q_{S} \in \Delta^{S}$,

$$
\begin{aligned}
p_{1} \succsim_{1} q_{1} & \Leftrightarrow\left\{\left(p_{1}, p_{S}\right) \succeq\left\{\left(q_{1}, p_{S}\right)\right\}\right. \\
& \Leftrightarrow \lambda\left\{\left(p_{1}, p_{S}\right)\right\}+(1-\lambda)\left\{\left(r_{1}, q_{1}\right)\right\} \succeq \lambda\left(q_{1}, p_{S}\right)+(1-\lambda)\left(r_{1}, q_{S}\right) \\
& \Leftrightarrow\left\{\left(\lambda p_{1}+(1-\lambda) l_{1}, \lambda p_{S}+(1-\lambda) q_{S}\right)\right\} \succeq\left\{\left(\lambda q_{1}+(1-\lambda) l_{1}, \lambda p_{S}+(1-\lambda) q_{S}\right)\right\} \\
& \Leftrightarrow \lambda p_{1}+(1-\lambda) r_{1} \succsim^{1} \lambda q_{1}+(1-\lambda) r_{1} .
\end{aligned}
$$


It is shown that $\succsim^{1}$ satisfies the axiom of Independence. In the same way, we can show that $\succsim^{S}$ satisfies the axiom of independence. We omit it.

By the von Neumann-Morgenstern's Expected Utility Theorem (see Kreps (1988) etc.), there exists a non-constant continuous and mixture linear function $u: \Delta \rightarrow \mathbb{R}$ which represents $\succsim^{1}$. Furthermore, $u$ is unique up to a positive affine transformation. Moreover, by Axiom 2 (Consistency), there exists a continuous and mixture linear utility function $\widehat{u}_{S}: \Delta^{S} \rightarrow \mathbb{R}$ with $\left(\alpha_{i}\right)_{i \in S}$ and a real number $\varepsilon$ such that $\widehat{u}_{S}\left(p_{S}\right)=\sum_{i \in S} u\left(p_{i}\right)+\varepsilon$ and $\sum_{i \in S} \alpha_{i}=1$. Define $u_{S}:=\sum_{i \in S} \alpha_{i} u\left(p_{i}\right)=\widehat{u}_{S}\left(p_{S}\right)-\varepsilon$. Hence, $u_{S}$ represents $\succsim^{S}$.

$\alpha_{1}$ is positive. Finally, we show $\alpha_{1}>0$. By Axiom 1 , define, for any $\boldsymbol{p} \in \Delta^{I}, V(\{\mathbf{p}\}):=$ $\sum_{i \in I} \alpha_{i} u\left(p_{i}\right)$ that represents $\succeq$ over $\mathcal{A}_{s}$, i.e., the set of all singletons. Suppose $\alpha_{1} \leq 0$. Take $\boldsymbol{p}, \boldsymbol{q} \in \Delta^{I}$ such that $p_{1} \succsim^{1} q_{1}$ and $p_{S} \sim^{S} q_{S}$. By Axiom 3 (Pareto), $\{\boldsymbol{p}\} \succ\{\boldsymbol{q}\}$. If $\alpha_{1} \leq 0$, $V(\{\boldsymbol{p}\}) \leq V(\{\boldsymbol{q}\}) \Leftrightarrow\{\boldsymbol{p}\} \preceq\{\boldsymbol{q}\}$. This is a contradiction.

\section{Step 2}

In Step 2, we show that $\left(\bar{u}_{1}, \bar{u}_{S}\right)$ in the second term of the RDIC represent $\left(\succeq_{1}, \succeq_{S}\right)$, respectively. For each $j \in\{1, S\}$, we show that $\succeq_{j}$ satisfies (i) completeness, (ii) transitivity, (iii) continuity, (iv) singleton independence, and (v) disjoint set-betweenness, by following from Olszewski (2007).

\section{Properties on $\succeq_{1}$ and $\succeq_{S}$}

Remember the definition of the induced menu-preferences. For each $j \in\{1, S\}$, we say that $A \succeq_{j} B$ if for any $\boldsymbol{q} \in B$ and $\boldsymbol{p} \in A, p_{j} \succsim^{j} q_{j}$.

Transitivity. We show that, for each $j \in\{1, S\}, \succeq_{j}$ is transitive. Take $A, B, C \in \mathcal{A}$ with $A \succeq_{j} B$ and $B \succeq_{j} C$. By definition, it is obvious that $A \succeq_{j} C$.

Completeness. We show that, for each $j \in\{1, S\}, \succeq_{j}$ is complete. By the axioms of Standard Preferences, Singleton Independence of $\succeq$, and Intrinsic Set-Betweenness of $\succeq_{j}$, for any $A \in \mathcal{A}$, there exists a singleton equivalent $\boldsymbol{p}_{A} \in \Delta^{I}$ such that $\left\{\boldsymbol{p}_{A}\right\} \sim A$. Consider arbitrary two menus $A, B \in \mathcal{A}$. Take singleton equivalent, respectively, denoted by $\boldsymbol{p}_{A}, \boldsymbol{p}_{B} \in$ $\Delta^{I}$. Without loss of generality, assume $\left\{\boldsymbol{p}_{A}\right\} \succeq_{j}\left\{\boldsymbol{p}_{B}\right\}$. We need to show that $A \succeq_{j} B \Leftrightarrow$ $\left\{\boldsymbol{p}_{A}\right\} \succeq_{j}\left\{\boldsymbol{p}_{B}\right\}$. The sufficiency part is obvious by definition. We show the necessity part. By the transitivity of $\succeq_{j}, A \sim_{j}\left\{\boldsymbol{p}_{A}\right\} \succeq_{j}\left\{\boldsymbol{p}_{B}\right\} \sim_{j} B$. Hence, $A \succeq_{j} B$. This argument holds for any arbitrary menus. 
Continuity. We show that, for each $j \in\{1, S\}, \succeq_{j}$ is continuous. Take arbitrary two allocations $\boldsymbol{p}, \boldsymbol{q} \in \Delta^{I}$ with $\{\boldsymbol{p}\} \succeq\{\boldsymbol{q}\}$. Suppose that $\{\boldsymbol{p}\} \succeq_{j}\{\boldsymbol{q}\}$. By definition, $p_{j} \succsim^{j} q_{j}$. Since $\succsim^{j}$ is continuous, the continuity of $\succeq_{j}$ holds on singletons. By taking singleton equivalent, we can show that the continuity of $\succeq_{j}$ holds for any arbitrary menus.

Disjoint Set-Betweenness. We show that, for each $j \in\{1, S\}, \succeq_{j}$ satisfies Disjoint SetBetweenness. This axiom is equivalent to Axiom 4 (Intrinsic Set-Betweenness).

Indifference to Randomization. We verify that $\succeq_{j}$ satisfies the axiom of Indifference to Randomization. The axiom is stated as follows. For each menu $A \in \mathcal{A}$, let $\operatorname{co}(A)$ be the convex hull of $A$.

Axiom 24. (Indifference to Randomization): For any $A \in \mathcal{A}, A \sim \operatorname{co}(A)$.

Take $A \in \mathcal{A} . \quad A$ is compact, and there exist $\succ^{j}$-best and $\succ^{j}$-worst allocations of the menu $A$. The extreme points do not change by objective mixtures. Hence, for all $A \in \mathcal{A}$, $A \sim \operatorname{co}(A)$.

We obtain the following result. For simplicity, let us introduce some notation: for each $p_{1} \in \Delta$ and $p_{S} \in \Delta^{I}, u_{1}:=\alpha_{1} u\left(p_{1}\right), u_{S}:=\sum_{i \in S} \alpha_{i} u\left(p_{i}\right)$.

Corollary 9. $\succeq_{j}$ satisfies Completeness, Transitivity, Continuity, Disjoint Set-Betweenness, and Indifference to Randomization, if and only if there exist a pair $\left(u, W_{j}\right)$ where $u: \Delta \rightarrow \mathbb{R}$ and $W_{j}: u(\Delta) \times u(\Delta) \rightarrow \mathbb{R}$ such that $\succeq_{j}$ is represented by $\bar{u}_{j}: \mathcal{A} \rightarrow \mathbb{R}$, defined by

$$
\bar{u}_{j}(A)=W_{j}\left(\max _{\boldsymbol{p} \in A} u\left(p_{j}\right), \min _{\boldsymbol{p} \in A} u\left(p_{j}\right)\right) .
$$

Proof. The proof mainly follows from Dekel et al. (2001) and Kopylov (2009). By Step 1, we have already shown that there exists a non-constant linear $u: \Delta \rightarrow \mathbb{R}$. The remaining is to show the existence of $W_{j}$. To prove it, we use the result of Kopylov (2009), and especially, we show that $\succeq_{j}$ satisfies Finiteness in Kopylov (2009).

Axiom 25. (Finiteness): For any sequence $\left\{A_{n}\right\}$ of $\mathcal{A}$, there exists a positive integer $N$ such that $\cup_{n=1}^{N} A_{n} \sim_{j} \cup_{n=1}^{N+1} A_{n}$.

To show that $\succeq_{j}$ satisfies Finiteness, take an arbitrary menu $A \in \mathcal{A}$. $A$ is compact, so there exist $\succ^{j}$-best and $\succ^{j}$-worst allocations. Let $\bar{p}_{j} \in A$ and $\underline{p}_{j} \in A$ respectively. Take a menu $B \in \mathcal{A}$ such that for any $\boldsymbol{q} \in B, \bar{p}_{j} \succsim^{j} q_{j} \succsim^{j} \underline{p}_{j}$. Then, $A \sim_{j} A \cup B$. Take $A_{1}, A_{2}, A_{3} \in \mathcal{A}$. Let $\overline{\boldsymbol{p}} \in \max _{\boldsymbol{p} \in A_{1} \cup A_{2} \cup A_{3}} u\left(p_{j}\right)$, and $\underline{\boldsymbol{p}} \in \min _{\boldsymbol{p} \in A_{1} \cup A_{2} \cup A_{3}} u\left(p_{j}\right)$. By construction, assume that $\overline{\boldsymbol{p}} \in A_{1}$ and $\underline{\boldsymbol{p}} \in A_{1}$. Suppose that $A_{1} \cup A_{2} \succeq_{j} A_{1} \cup A_{3}$. Then, by definition, $A_{1} \cup A_{2} \sim$ $A_{1} \cup A_{2} \cup A_{3}$. Thus, Finiteness is satisfied with $N=2$. By Intrinsic Set-Betweenness in the above argument, we obtain the support of the sign that is max $u_{j}$ and $\min u_{j}$. By Theorem 1.A (a weak EU representaion) in Dekel et al. (2001), there exists $W_{j}: u(\Delta) \times u(\Delta) \rightarrow \mathbb{R}$, which describes the desired representation. 
Singleton Independence. We show that, for each $\succeq_{j}$ satisfies Singleton Independence, to obtain the desired result. This follows from Axiom 6 (Singleton Independence) of $\succeq$.

We obtain the following result.

Corollary 10. $\succeq_{j}$ satisfies Completeness, Transitivity, Continuity, Disjoint Set-Betweenness, and Singleton Independence, if and only if there exist a pair $\left(u, \gamma_{j}\right)$ where $u: \Delta \rightarrow \mathbb{R}$ and $\gamma_{j} \in[0,1]$ such that $\succeq_{j}$ is represented by $\bar{u}_{j}: \mathcal{A} \rightarrow \mathbb{R}$, defined by

$$
\bar{u}_{j}(A)=\gamma_{j} \max _{\boldsymbol{p} \in A} u\left(p_{j}\right)+\left(1-\gamma_{j}\right) \min _{\boldsymbol{p} \in A} u\left(p_{j}\right) .
$$

Proof. The result follows from Theorem 1 in Olszewski (2007).

By putting $u_{1}:=\alpha_{1} u\left(p_{1}\right)$ and $u_{S}:=\sum_{i \in S} \alpha_{i} u\left(p_{i}\right)$ for each $p_{1} \in \Delta$ and $p_{S} \in \Delta^{S}$, we obtain the desired utility representation on reference-point formation; for any $A, B \in \mathcal{A}$, $A \succeq_{j} B \Leftrightarrow \bar{u}_{j}(A)=\bar{u}_{j}(B)$.

\section{Step 3}

In Step 3, we introduce a binary relation $\succeq^{*}$ on the set of menus $\mathcal{A}^{*}$ defined later. We show that $V^{*}: \mathcal{A}^{*} \rightarrow \mathbb{R}$ represents $\succeq *$.

Utility Space. We consider a set of utilities of allocations on a utility space in each menu $A$. For any $A \in \mathcal{A}$, define

$$
\boldsymbol{u}(A):=\left\{\left(u_{1}\left(p_{1}\right), u_{S}\left(p_{S}\right)\right) \in \mathbb{R}^{2} \mid \boldsymbol{p}=\left(p_{1}, p_{S}\right) \in A\right\},
$$

where $u_{1}\left(p_{1}\right)=\alpha_{1} u\left(p_{1}\right)$ and $u_{S}\left(p_{S}\right)=\sum_{i \in S} \alpha_{i} u\left(p_{i}\right)$. Let us $\mathcal{A}^{*}$ be denoted by $\{\boldsymbol{u}(A) \mid A \in$ $\mathcal{A}\}$. Since each choice set $A \in \mathcal{A}$ is compact, $\boldsymbol{u}(A)$ is also compact, by the continuity of $u: \Delta \rightarrow \mathbb{R}$. By the uniqueness property of $u$, we can normalize $u(\Delta)=[0,1]$ and $u_{S}\left(\Delta^{S}\right)=[0,1] . \mathcal{A}^{*}$ is a set of compact subsets of $[0,1]^{2}$, endowed with the Hausdorff metric. Define $\succeq{ }^{*}$ on $\mathcal{A}^{*}$ in the following way.

Definition 16. For any $A^{*}, B^{*} \in \mathcal{A}^{*}$,

$$
A^{*} \succeq^{*} B \text { if } A \succeq B,
$$

where $A^{*}=\boldsymbol{u}(A)$ and $B^{*}=\boldsymbol{u}(B)$.

The asymmetric and symmetric parts of $\succeq^{*}$ are denoted by $\succ^{*}$ and $\sim^{*}$, respectively. 
$\succeq^{*}$ is well-defined. First of all, we show that $\succeq^{*}$ is well-defined.

Lemma 1. $\succeq^{*}$ is well-defined.

Proof. Suppose $A^{*}=B^{*}$, i.e., $\boldsymbol{u}(A)=\boldsymbol{u}(B)$. We need to show that $A \sim B$. Then, for any $\boldsymbol{p} \in A$ there exists $\boldsymbol{q} \in B$ such that $p_{1} \sim_{1} q_{1}$ and $p_{S} \sim_{S} q_{S}$. Hence, $A \sim_{1} B$ and $A \sim_{S} B$ hold. By Axiom 7 (Weak Dominance), we have $A \sim A \cup B$. In the same way, we have $B \sim A \cup B$. By Axiom 1 (Standard Preferences), in particular, the transitivity of $\succeq$, we obtain $A \sim B$.

Axioms. Consider the axioms in Theorem 1 in the above utility space. We show that $\succeq^{*}$ satisfies the following axioms. Let us introduce $\succeq_{j}^{*}$ on $\mathcal{A}^{*}$ for each $j \in\{1, S\}$. We say that for each $j \in\{1, S\}, A^{*} \succeq_{j}^{*} B^{*}$ if for any $\boldsymbol{v} \in B^{*}$ and $\boldsymbol{u} \in A^{*}$ such that $u_{j} \geq v_{j}$.

Axiom* 1. (Pareto*): For any $\boldsymbol{u}, \boldsymbol{v} \in[0,1]^{2}$, if $\boldsymbol{u} \geq \boldsymbol{v}$, then $\{\boldsymbol{u}\} \succeq\{\boldsymbol{v}\}$.

Axiom* 2. (Weak Dominance*): For any $A^{*}, B^{*} \in \mathcal{A}^{*}$, if $A^{*} \sim_{1}^{*} B^{*}, A^{*} \sim_{S}^{*} B^{*}$, and $A^{*} \succeq^{*} B^{*}$, then $A^{*} \sim^{*} A^{*} \cup B^{*}$.

Axiom* 3. (Intrinsic Set-Betweenness*): For each $j \in\{1, S\}$, if $A^{*} \cap B^{*}=\varnothing$ and $A^{*} \succeq_{j}^{*} B^{*}$, then $A^{*} \succeq_{j}^{*} A^{*} \cup B^{*} \succeq_{j}^{*} B$.

Axiom* 4. (Singleton Independence*): For any $A^{*}, B^{*} \in \mathcal{A}^{*}, \boldsymbol{w} \in[0,1]^{2}$, and $\lambda \in[0,1]$

$$
A^{*} \succeq^{*} B^{*} \Rightarrow \lambda A^{*}+(1-\lambda)\{\boldsymbol{w}\} \succeq^{*} \lambda B^{*}+(1-\lambda)\{\boldsymbol{w}\}
$$

Axiom* 5. (Weak Independence*): or any $A^{*}, B^{*}, C^{*} \in \mathcal{A}^{*}$ and $\lambda \in[0,1]$,

$$
(\forall j \in\{1, S\}) A^{*} \succeq_{j}^{*} B^{*} \Rightarrow \lambda A^{*}+(1-\lambda) C^{*} \succeq^{*} \lambda B^{*}+(1-\lambda) C^{*} .
$$

We obtain the following result.

Lemma 2. $\succeq^{*}$ is a continuous weak order that satisfies Paret*, Weak Dominance*, Intrinsic Set-Betweenness*, Singleton Independence*, and Weak Independence*

Proof. We omit the proof, since we suppose that $\succeq$ satisfies the axioms in Theorem 1. By the definition of $\succeq^{*}$, it is easily verified that $\succeq^{*}$ satisfies the axioms in the utility space.

\section{Step 4}

In Step 4, we complete the utility representation of the RDIC utilitarian. First, we identify a finite subjective state space, by following from Kopylov (2009). Next, we show that $V^{*}$ has a functional form of the subjective state space (Lemma 4). 
A Utility Representation of a Subjective State Space. For each menu $A^{*}$ in the utility space $\mathcal{A}^{*}$, let $\overline{\boldsymbol{u}}_{A^{*}}$ be the reference point of the menu $A^{*}$. Let $\bar{u}_{1}=\gamma_{1} \max u_{1}+\left(1-\gamma_{1}\right) \min u_{1}$ where $\gamma_{1} \in[0,1]$, and $\bar{u}_{S}=\gamma_{S} \max u_{S}+\left(1-\gamma_{S}\right)$ min $u_{S}$ where $\gamma_{S} \in[0,1]$.

Suppose that $\succeq^{*}$ is a continuous weak order that satisfies Axioms* $1-5$ in Step 3.

Definition 17. There exists a unique function $\mu:[0,1]^{2} \times \mathbb{R} \rightarrow \mathbb{R}$ such that $\succeq^{*}$ on $\mathcal{A}^{*}$ is represented by $V^{*}: \mathcal{A}^{*} \rightarrow \mathbb{R}$ defined by

$$
V^{*}\left(A^{*}\right)=\sum_{\lambda \in \mathbb{R}} \mu\left(\overline{\boldsymbol{u}}_{A^{*}}, \lambda\right)\left(\max _{\boldsymbol{u} \in A^{*}} \lambda \bar{u}_{1}+(1-\lambda) \bar{u}_{S}\right) .
$$

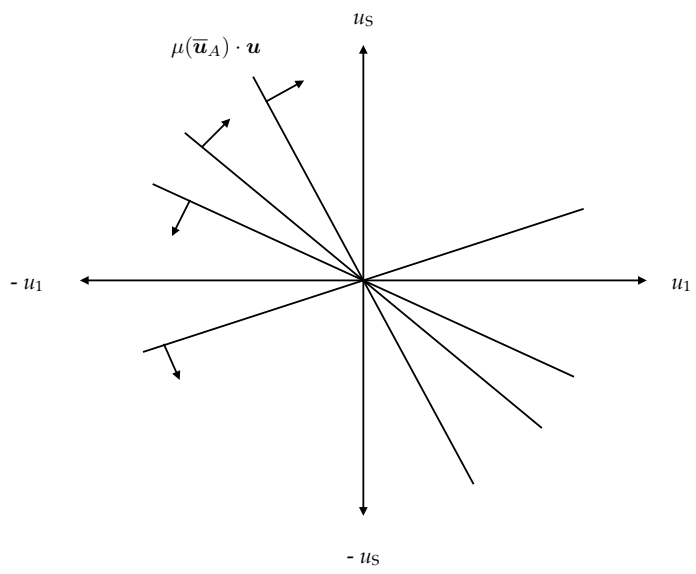

Figure 6: Definition 17: Utility Representation with a Subjective State Space

We have defined $\mu:[0,1]^{2} \times \mathbb{R} \rightarrow \mathbb{R}$. The first argument is a reference point, so $\mu$ depends on reference points. Even though we relax Independence, we still have an additive utility representation. The additivity follows from Axiom* 5 (Weak Independence*). By Axiom 5, the sign of $\mu$ depends on the reference point $\overline{\boldsymbol{u}}$. To obtain the desired functional form, we show the following. First, we show that $\succeq^{*}$ satisfies Finiteness* in Kopylov (2009).

Axiom* 6. (Finiteness*): For any sequence $\left\{A_{n}^{*}\right\}$ of $\mathcal{A}^{*}$, there exists a positive integer $N$ such that $\cup_{n=1}^{N} A_{n}^{*} \sim \cup_{n=1}^{N+1} A_{n}^{*}$.

Lemma 3. $\succeq^{*}$ satisfies Finiteness (Axiom 6).

Proof. We show the following: Take arbitrary two menus $A^{*}, B^{*} \in \mathcal{A}$ satisfying the following: for any $\boldsymbol{v} \in B^{*}$ and $\boldsymbol{u} \in A^{*}, u_{1} \geq v_{1}$ and $u_{S} \geq v_{S}$. We obtain $A^{*} \succeq^{*} B^{*}$. This implies that, for any $\boldsymbol{v} \in A^{*} \cup B^{*}$, there exists $\boldsymbol{u} \in A^{*}$ such that $u_{1}=v_{1}$ and $u_{S}=v_{S}$. Then, by Axiom 2 (Weak Dominance $)^{*}$, we have $A^{*} \sim A^{*} \cup B^{*}$.

Take arbitrary menus $A_{1}^{*}, A_{2}^{*}, A_{3}^{*}, A_{4}^{*} \in \mathcal{A}^{*}$. Let us denote the following: 


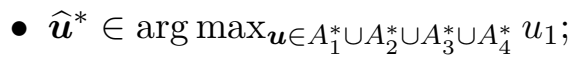

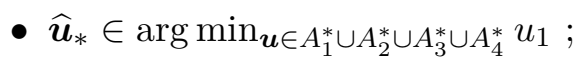

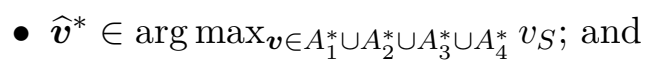

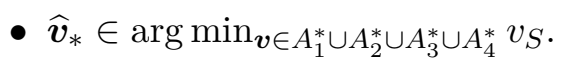

Without loss of generality, assume $\widehat{\boldsymbol{u}}^{*}, \widehat{\boldsymbol{u}}_{*} \in A_{1}^{*}$ and $\widehat{\boldsymbol{v}}^{*}, \widehat{\boldsymbol{v}}_{*} \in A_{2}^{*}$. Suppose $A_{1}^{*} \cup A_{2}^{*} \cup A_{3}^{*} \succeq^{*}$ $A_{1}^{*} \cup A_{2}^{*} \cup A_{4}^{*}$. Then, by Axiom 2 (Weak Dominance ${ }^{*}$ ), we have $A_{1}^{*} \cup A_{2}^{*} \cup A_{3}^{*} \sim^{*} A_{1}^{*} \cup A_{2}^{*} \cup A_{3}^{*} \cup A_{4}^{*}$. Hence, Axiom 6 (Finiteness ${ }^{*}$ ) is satisfied with $N=3$.

Identifying a Subjective State Space. We show the following lemma to obtain the desired representation.

Lemma 4. Suppose that $\succeq *$ is a continuous weak order that satisfies Axioms* $2-5$ in Step 3. Then, there exists at least one $\lambda^{*} \in(0,1)$ such that $\succeq^{*}$ is represented by

$$
\begin{gathered}
V^{*}\left(A^{*}\right)=\mu\left(\overline{\boldsymbol{u}}_{A^{*}}, 1\right) \max _{\boldsymbol{u} \in A^{*}} \bar{u}_{1}+\mu\left(\overline{\boldsymbol{u}}_{A^{*}}, \lambda^{*}\right) \max _{\boldsymbol{u} \in A^{*}}\left(\lambda^{*} \bar{u}_{1}+\left(1-\lambda^{*}\right) \bar{u}_{S}\right) \\
+\mu\left(\overline{\boldsymbol{u}}_{\left.A^{*}, 0\right)} \max _{\boldsymbol{u} \in A^{*}} \bar{u}_{S} .\right.
\end{gathered}
$$

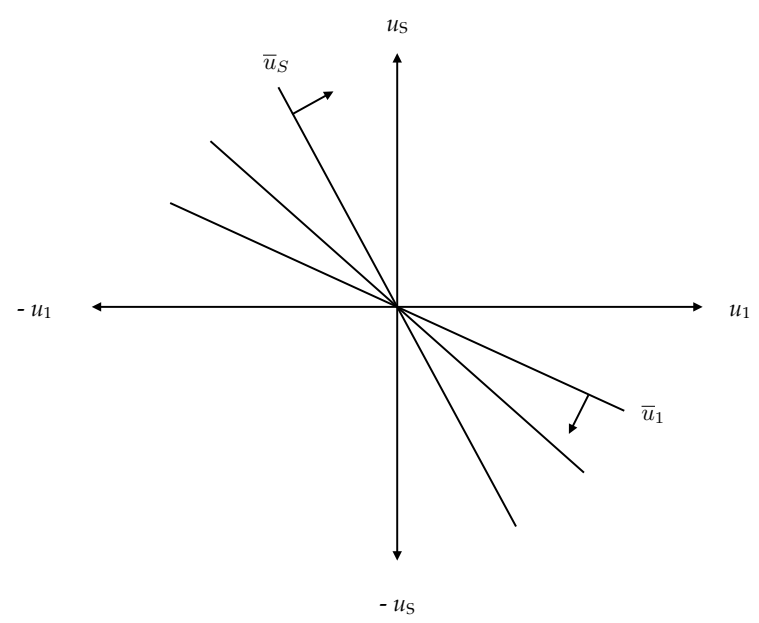

Figure 7: Lemma 4: Identification of a Subjective State Space

Proof. Remember that we can normalize $u(\Delta)=[0,1]$ and $u_{S}\left(\Delta^{I}\right)=[0,1]$. Fix $\varepsilon<\frac{1}{2}$. For all $\lambda \in \mathbb{R}$, define $\boldsymbol{u}^{*}(\lambda)=\left(u_{1}^{*}(\lambda), u_{S}^{*}(\lambda)\right)$ by

$$
u_{1}^{*}(\lambda)=\frac{1}{2}+\frac{\varepsilon \lambda}{\|(\lambda, 1-\lambda)\|},
$$


and

$$
u_{S}^{*}(\lambda)=\frac{1}{2}+\frac{\varepsilon(1-\lambda)}{\|(\lambda, 1-\lambda)\|} .
$$

First, we show that for all $\lambda \in[0,1], u_{1}^{*}(1) \geq u_{1}^{*}(\lambda)$ and $u_{S}^{*}(0) \geq u_{S}^{*}(\lambda)$. For all $\lambda \in[0,1]$, $u_{1}^{*}(\lambda)=\frac{1}{2}+\varepsilon \geq u_{1}^{*}(\lambda)$. In the same way, for all $\lambda \in[0,1], u_{S}^{*}(\lambda)=\frac{1}{2}+\varepsilon \geq u_{S}^{*}(\lambda)$.

Next, we show the following:

(i) for all $\lambda \notin[0,1], \mu\left(\overline{\boldsymbol{u}}\left(A^{*}\right), \lambda\right)=0$,

(ii) for all $\lambda \notin\{0,1\}, \mu\left(\overline{\boldsymbol{u}}\left(A^{*}\right), \lambda\right) \geq 0$, and

(iii) there exists a unique $\lambda \notin\{0,1\}, \mu\left(\overline{\boldsymbol{u}}\left(A^{*}\right), \lambda\right)>0$.

Proof of (i). We show (i) by the way of contradiction. Suppose that there exists $\lambda^{\prime} \notin[0,1]$ such that $\mu\left(\overline{\boldsymbol{u}}_{A^{*}}, \lambda^{\prime}\right) \neq 0$. Without loss of generality, suppose $\mu\left(\overline{\boldsymbol{u}}_{A^{*}}, \lambda^{\prime}\right)<0$. Consider the following menus:

- $A^{*}=\left\{\boldsymbol{u}^{*}(\lambda) \in[0,1]^{2} \mid \lambda \in\left\{0,1, \frac{\left|\lambda^{\prime}\right|}{\left|\lambda^{\prime}\right|+\left|1-\lambda^{\prime}\right|}\right\} \backslash\left\{\lambda^{\prime}\right\}\right\}$;

- $A^{*} \cup\left\{\boldsymbol{u}^{*}\left(\lambda^{\prime}\right)\right\}$.

Notice that the two menus are compact. Since $\boldsymbol{u}^{*}\left(\frac{\left|\lambda^{\prime}\right|}{\left|\lambda^{\prime}\right|+\left|1-\lambda^{\prime}\right|}\right) \in A^{*}$, and $u_{1}\left(\frac{\left|\lambda^{\prime}\right|}{\left|\lambda^{\prime}\right|+\left|1-\lambda^{\prime}\right|}\right) \geq$ $u_{1}\left(\lambda^{\prime}\right)$ and $u_{S}\left(\frac{\left|\lambda^{\prime}\right|}{\left|\lambda^{\prime}\right|+\left|1-\lambda^{\prime}\right|}\right) \geq u_{S}\left(\lambda^{\prime}\right)$, by Axiom 2 (Weak Dominance $), A^{*} \sim^{*} A^{*} \cup\left\{\boldsymbol{u}\left(\lambda^{\prime}\right)\right\}$.

If $\mu\left(\overline{\boldsymbol{u}}_{A^{*}}, \lambda^{\prime}\right)<0$ and $\mu\left(\overline{\boldsymbol{u}}_{A^{*} \cup\left\{\boldsymbol{u}\left(\lambda^{\prime}\right)\right\}}, \lambda^{\prime}\right)<0$, then $V^{*}\left(A^{*}\right)>V^{*}\left(A^{*} \cup\left\{\boldsymbol{u}\left(\lambda^{\prime}\right)\right\}\right)$, which represents $A^{*} \succ^{*} A^{*} \cup\left\{\boldsymbol{u}\left(\lambda^{\prime}\right)\right\}$. This is a contradiction.

Proof of (ii). We show (ii) by the way of contradiction. Suppose that there exists $\lambda \notin\{0,1\}$ such that $\mu(\lambda)<0$. Consider the following menus:

- $A^{*}=\left\{\boldsymbol{u}^{*}(\lambda) \in[0,1]^{2} \mid \lambda \in\left\{0, \frac{1}{2}, 1\right\}\right\}$

- $A^{*} \cup\left\{\boldsymbol{u}^{*}\left(\lambda^{\prime}\right)\right\}$.

The two menus are compact. Since $\boldsymbol{u}\left(\frac{1}{2}\right) \in A^{*}$, by Axiom 2 (Weak Dominance $\left.{ }^{*}\right), A^{*} \sim^{*}$ $A^{*} \cup\left\{\boldsymbol{u}\left(\lambda^{\prime}\right)\right\}$. However, by the assumption of $\mu\left(\cdot, \lambda^{\prime}\right)<0$, we have $V^{*}\left(A^{*}\right)>V^{*}\left(A^{*} \cup\left\{\boldsymbol{u}\left(\lambda^{\prime}\right)\right\}\right)$, which represents $A^{*} \succ^{*} A^{*} \cup\left\{\boldsymbol{u}\left(\lambda^{\prime}\right)\right\}$. This is a contradiction.

Proof of (iii). We show (iii). To see this, fix a menu $A^{*} \in \mathcal{A}^{*}$, and assume that there exists no $\lambda \notin(0,1)$ such that $\mu\left(\overline{\boldsymbol{u}}_{A}, \lambda\right)=0$. By (ii), for all $\lambda \in(0,1), \mu\left(\overline{\boldsymbol{u}}_{A}, \lambda\right)=0$. Take another menu $B^{*} \in \mathcal{A}^{*}$ such that for each $j \in\{1, S\}, A^{*} \sim_{j}^{*} B^{*}$, and $A^{*} \succ^{*} B^{*}$. By definition, under the assumption, we have $V^{*}\left(A^{*}\right)=V^{*}\left(B^{*}\right)$. Hence, to obtain $V^{*}\left(A^{*}\right)>V^{*}\left(B^{*}\right)$, there exists $\lambda \in(0,1)$ such that $\mu\left(\overline{\boldsymbol{u}}_{A}, \lambda\right)>0$. The uniqueness follows from the fact that $V^{*}$ is mixture-linear: for any $\sigma \in[0,1], V^{*}\left(\sigma A^{*}+(1-\sigma) B^{*}\right)=\sigma V^{*}\left(A^{*}\right)+(1-\sigma) V^{*}\left(B^{*}\right)$. 
Reference-Dependent Image-Conscious Utility Representation. By Lemma 4, we find three states, i.e., $\bar{u}_{1}, \lambda \bar{u}_{1}+(1-\lambda) \bar{u}_{S}$, and $\bar{u}_{S}$. We normalize $\mu$ in the following way. First, let $\left(2 \gamma_{1}-1\right) \mu\left(\overline{\boldsymbol{u}}_{A}, \lambda^{*}\right) \lambda^{*}+\mu\left(\overline{\boldsymbol{u}}_{A}, 1\right)=1$. Define, for each menu $A^{*} \in \mathcal{A}^{*}, \beta_{1}\left(\overline{\boldsymbol{u}}_{A}\right):=-\mu\left(\overline{\boldsymbol{u}}_{A}, 1\right)$. Second, in the same way, normalize $\mu$ as follows: $\left(2 \gamma_{S}-1\right) \mu\left(\overline{\boldsymbol{u}}_{A}, \lambda^{*}\right)\left(1-\lambda^{*}\right)+\mu\left(\overline{\boldsymbol{u}}_{A}, 0\right)=1$. Let $\beta_{S}\left(\overline{\boldsymbol{u}}_{A}\right):=\mu\left(\overline{\boldsymbol{u}}_{A}, 0\right)$. Then, we have the utility representation in the following way (Figure 8): for any $A^{*} \in \mathcal{A}^{*}$,

$$
\begin{array}{r}
V^{*}\left(A^{*}\right)=\max _{\boldsymbol{u} \in A^{*}}\left(1+\beta_{1}\left(\overline{\boldsymbol{u}}_{A}\right)\right) \alpha_{1} u_{1}+\left(1-\beta_{S}\left(\overline{\boldsymbol{u}}_{A}\right)\right) u_{S} \\
-\beta_{1}\left(\overline{\boldsymbol{u}}_{A}\right) \max _{\boldsymbol{u} \in A^{*}} \bar{u}_{1} \\
+\beta_{S}\left(\overline{\boldsymbol{u}}_{A}\right) \max _{\boldsymbol{u} \in A^{*}} \bar{u}_{S} .
\end{array}
$$

By the axiom of Pareto* $^{*}$, we obtain $\beta_{1}\left(\overline{\boldsymbol{u}}_{A^{*}}\right) \in(-1, \infty)$ for any $A^{*} \in \mathcal{A}^{*}$. In the same way, we obtain $\beta_{S}\left(\overline{\boldsymbol{u}}_{A^{*}}\right) \in(-\infty, 1)$ for any $A^{*} \in \mathcal{A}^{*}$.

For any $A \in \mathcal{A}$, define $V(A)=V^{*}\left(A^{*}\right)$. Then, we have

$$
\begin{aligned}
A \succeq B & \Leftrightarrow A^{*} \succeq^{*} B^{*} \\
& \Leftrightarrow V^{*}\left(A^{*}\right) \geq V^{*}\left(B^{*}\right) \\
& \Leftrightarrow V(A) \geq V(B) .
\end{aligned}
$$

By letting $u_{1}=\alpha_{1} u\left(p_{1}\right)$ and $u_{S}=\sum_{i \in S} \alpha_{i} u\left(p_{i}\right)$, we have the desired representation; that is, for any $A \in \mathcal{A}$,

$$
\begin{aligned}
V(A)=\max _{\boldsymbol{p} \in A}\left[\sum_{i \in I} \alpha_{i} u\left(p_{i}\right)\right. & +\beta_{1}\left(\overline{\boldsymbol{u}}_{A}\right)\left(\alpha_{1}\left(u\left(p_{1}\right)-\bar{u}_{1}(A)\right)\right) \\
& \left.-\beta_{S}\left(\overline{\boldsymbol{u}}_{A}\right)\left(\sum_{i \in S} \alpha_{i} u\left(p_{i}\right)-\bar{u}_{S}(A)\right)\right] .
\end{aligned}
$$

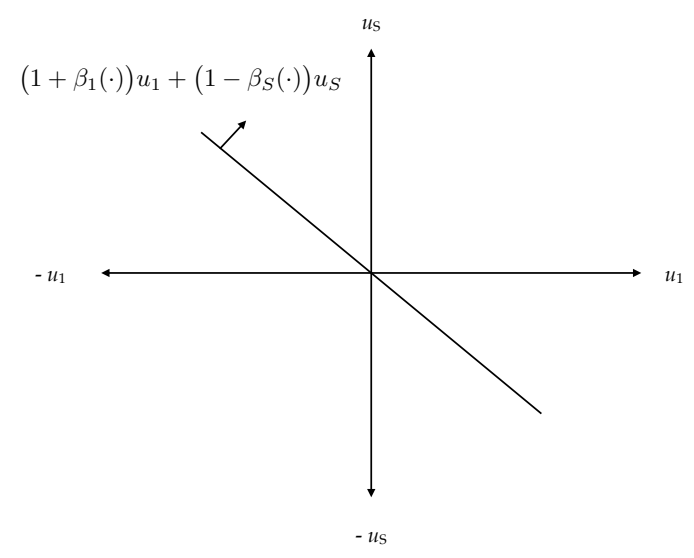

Figure 8: Reference-Dependent Image-Conscious Utilitarian

We complete the proof of the sufficiency part. 


\section{A.2 Necessity Part}

We show the necessity part. We show that the utility representation satisfies the axiom of Weak Dominance and Intrinsic Set-betweenness. It is easy to prove the necessity of the other axioms.

Weak Dominance. First, to show Axiom 5 (Weak Dominance), take arbitrary two menus $A, B \in \mathcal{A}$ with $A \sim_{1} B, A \sim_{S} B$, and $A \succeq B$. Consider a RDIC with a four-tuple $(u, \boldsymbol{\alpha}, \boldsymbol{\beta}, \boldsymbol{\gamma})$; that is, for any $A \in \mathcal{A}$,

$$
\begin{aligned}
V(A)=\max _{\boldsymbol{p} \in A}\left[\sum_{i \in I} \alpha_{i} u\left(p_{i}\right)\right. & +\beta_{1}\left(\overline{\boldsymbol{u}}_{A}\right)\left(\alpha_{1}\left(u\left(p_{1}\right)-\bar{u}_{1}(A)\right)\right) \\
& \left.-\beta_{S}\left(\overline{\boldsymbol{u}}_{A}\right)\left(\sum_{i \in S} \alpha_{i} u\left(p_{i}\right)-\bar{u}_{S}(A)\right)\right] .
\end{aligned}
$$

Let $\sum_{i \in I} \alpha_{i} u\left(p_{i}\right), \beta_{1}\left(\overline{\boldsymbol{u}}_{A}\right)\left(\alpha_{1}\left(u\left(p_{1}\right)-\bar{u}_{1}(A)\right)\right),-\beta_{S}\left(\overline{\boldsymbol{u}}_{A}\right)\left(\sum_{i \in S} \alpha_{i} u\left(p_{i}\right)-\bar{u}_{S}(A)\right)$ be the first term, the second term, and the third term, respectively. For each $A \in \mathcal{A}$, let $\Delta_{1}(A):=$ $\alpha_{1}\left(u\left(p_{1}\right)-\bar{u}_{1}(A)\right)$ where $\boldsymbol{p}$ is a maximizer in $A$, and $\left.\Delta_{S}(A):=u\left(p_{S}\right)-\bar{u}_{S}(A)\right)$ where $u\left(p_{S}\right):=$ $\sum_{i \in S} \alpha_{i} u\left(p_{i}\right)$ and $\boldsymbol{p}$ is a maximizer in $A$.

Consider the menu $A \cup B$. Since $A \succeq B, \boldsymbol{p} \in A$ is a maximizer in $A \cup B$. Then, $\Delta_{1}(A)=\Delta_{1}(A \cup B)$ and $\Delta_{S}(A)=\Delta_{S}(A \cup B)$ hold. Moreover, since $A \sim_{1} B$ and $A \sim_{S} B$ hold, $\overline{\boldsymbol{u}}_{A}=\overline{\boldsymbol{u}}_{A \cup B}$. Then, $\boldsymbol{\beta}(A)=\boldsymbol{\beta}(A \cup B)$. Thus, the utility function has the same terms. Hence, $V(A)=V(A \cup B) \Leftrightarrow A \sim A \cup B$.

Intrinsic Set-Betweenness. Next, we show that $V$ represents $\succeq$ that satisfies Axiom 4 (Intrinsic Set-Betweenness). Consider $j \in\{1, S\}$. Suppose that $A \cap B=\varnothing$ and $A \succeq_{j} B$. Then, by $\gamma_{j} \in[0,1]$ and the definition of $\bar{u}_{j}$, we have $\bar{u}_{j}(A) \geq \bar{u}_{j}(A \cup B)$ and $\bar{u}_{j}(A \cup B) \geq$ $\bar{u}_{j}(B)$. Hence, $\succeq_{j}$ satisfies the axiom of Intrinsic Set-betweenness. 


\section{B Proof of Propositions}

\section{B.1 Proof of Proposition 1}

(i) and (ii). The uniqueness result is easily obtained. We show (i) and (ii). By the standard uniqueness result of the expected utility theorem (EUT), we obtain $\alpha_{i}=\alpha_{i}^{\prime}$ for all $i \in I$. Moreover, there exists $a>0$ and $b \in \mathbb{R}$ such that $u^{\prime}=a u+b$.

(iv). Next, we show (iv). It is straightforward to prove $\gamma=\gamma^{\prime}$. Consider $j \in\{1, S\}$. By (i), $\bar{u}_{j}=a \bar{u}_{j}^{\prime}+b$ for some $a>0$ and $b \in \mathbb{R}$. Let $\widehat{u}_{j}^{*}:=\max _{\boldsymbol{p} \in A} u_{j}, \widehat{u}_{* j}:=\min _{\boldsymbol{p} \in A} u_{j}$, $\widehat{u}_{j}^{* *}:=\max _{\boldsymbol{p} \in A} u_{j}^{\prime}=u_{j}^{\prime *}$, and $\widehat{u}_{* j}^{\prime}:=\min _{\boldsymbol{p} \in A} u_{j}^{\prime}$ for each $j \in\{1, S\}$. Notice that $u_{1}=\alpha_{1} u\left(p_{1}\right)$ and $u_{S}=\sum_{i \in S} \alpha_{i} u\left(p_{i}\right)$ for all $\boldsymbol{p} \in \Delta^{I}$. Then, $\bar{u}_{j}=\gamma_{j} \widehat{u}_{j}^{*}+\left(1-\gamma_{j}\right) \widehat{u}_{* j}=\gamma_{j}\left(a \widehat{u}_{j}^{* *}+b\right)+(1-$ $\left.\gamma_{j}\right)\left(a \widehat{u}_{* j}+b\right)=a\left[\gamma_{j} \widehat{u}_{j}^{*}+\left(1-\gamma_{j}\right) \widehat{u}_{* j}^{\prime}\right]+b$. Since $\bar{u}_{j}=a \bar{u}_{j}^{\prime}+b$, it must be $\gamma_{j}=\gamma_{j}^{\prime}$. Hence, $\gamma=\gamma^{\prime}$.

(iii). Finally, we show (iii). By (i) and the proof of Theorem 1 , note that $V^{*}=a V^{*}+b \Leftrightarrow$ $V=a V^{\prime}+b$. The ex-post utility $U$ is given by $U=\left(1-\beta_{1}^{*}\right) \alpha_{1} u_{1}+\left(1+\beta_{S}^{*}\right) \sum_{i \in S} \alpha_{i} u_{i}$. By (i), (ii), and (iv), $U=a U^{\prime}+b$. In the similar way, we have $\boldsymbol{\beta}(\cdot)=\boldsymbol{\beta}^{\prime}(\cdot)$.

\section{B.2 Proof of Proposition 2}

the Necessity Part. First, we show the necessity part. We show that the decision maker $X$ is more altruistic than the decision maker $Y$. Suppose $\alpha_{1}^{X} \leq \alpha_{1}^{Y}$. Take two allocations $\boldsymbol{p}, \boldsymbol{q} \in \Delta^{I}$ such that $p_{S} \succ_{S}^{h} q_{S}$ for each $h \in\{X, Y\}$. By definition, suppose $\{\boldsymbol{p}\} \succeq^{Y}\{\boldsymbol{q}\}$. Since $p_{S} \succ_{S}^{h} q_{S}$, we have $\sum_{i \in S} \alpha_{i} u\left(p_{i}\right)>\sum_{i \in S} \alpha_{i} u\left(q_{i}\right)$. We consider the two cases: (i) $u\left(p_{1}\right) \geq u\left(q_{1}\right)$, and (ii) $u\left(p_{1}\right)<u\left(q_{1}\right)$.

Consider the case $u\left(p_{1}\right) \geq u\left(q_{1}\right)$. By definition, $\alpha_{1}^{X}>0$. Then, we have $\alpha_{1}^{X} u\left(p_{1}\right)+$ $\sum_{i \in S} \alpha_{i} u\left(p_{i}\right) \geq \alpha_{1}^{X} u\left(q_{1}\right)+\sum_{i \in S} \alpha_{i} u\left(q_{i}\right)$. Thus, $\{\boldsymbol{p}\} \succeq^{X}\{\boldsymbol{q}\}$, which implies that $X$ is more altruistic than $Y$.

Consider the case $u\left(p_{1}\right)<u\left(q_{1}\right)$. Remember that $\{\boldsymbol{p}\} \succeq^{Y}\{\boldsymbol{q}\}$. Then, the following must hold: $\alpha_{1}^{Y}\left(u\left(p_{1}\right)-u\left(q_{1}\right)\right) \geq \sum_{i \in S} \alpha_{i} u\left(q_{i}\right)-\sum_{i \in S} \alpha_{i} u\left(p_{i}\right)$. We have $u\left(p_{1}\right)-u\left(q_{1}\right)<0$ and we suppose $\alpha_{1}^{X} \leq \alpha_{1}^{Y}$, so $\alpha_{1}^{X}\left(u\left(p_{1}\right)-u\left(q_{1}\right)\right) \geq \sum_{i \in S} \alpha_{i} u\left(q_{i}\right)-\sum_{i \in S} \alpha_{i} u\left(p_{i}\right)$. Hence, $\alpha_{1}^{X} u\left(p_{1}\right)+\sum_{i \in S} \alpha_{i} u\left(p_{i}\right) \geq \alpha_{1}^{X} u\left(q_{1}\right)+\sum_{i \in S} \alpha_{i} u\left(q_{i}\right)$. Thus, $\{\boldsymbol{p}\} \succeq^{Y}\{\boldsymbol{q}\}$, which implies that $X$ is more altruistic than $Y$.

the Sufficiency Part. Next, we show the sufficiency part. Take two allocations $\boldsymbol{p}, \boldsymbol{q} \in \Delta^{I}$ such that $p_{S} \succ_{S}^{h} q_{S}$ and $p_{1} \succ_{1}^{h} q_{1}$, for each $h \in\{X, Y\}$. Without loss of generality, suppose $\{\boldsymbol{p}\} \sim^{Y}\{\boldsymbol{q}\}$. Then, we have $\alpha_{1}^{Y} u\left(p_{1}\right)+\sum_{i \in S} \alpha_{i} u\left(p_{i}\right)=\alpha_{1}^{Y} u\left(q_{1}\right)+\sum_{i \in S} \alpha_{i} u\left(q_{i}\right)$. We obtain

$$
\alpha_{1}^{Y}=\frac{\sum_{i \in S} \alpha_{i} u\left(p_{i}\right)-\sum_{i \in S} \alpha_{i} u\left(q_{i}\right)}{u\left(q_{1}\right)-u\left(p_{1}\right)} .
$$


Suppose that $X$ is more altruistic than $Y$. Then, $\{\boldsymbol{p}\} \succeq^{X}\{\boldsymbol{q}\}$. We obtain $\alpha_{1}^{X} u\left(p_{1}\right)+$ $\sum_{i \in S} \alpha_{i} u\left(p_{i}\right) \geq \alpha_{1}^{X} u\left(q_{1}\right)+\sum_{i \in S} \alpha_{i} u\left(q_{i}\right)$. Hence,

$$
\frac{\sum_{i \in S} \alpha_{i} u\left(p_{i}\right)-\sum_{i \in S} \alpha_{i} u\left(q_{i}\right)}{u\left(q_{1}\right)-u\left(p_{1}\right)} \geq \alpha_{1}^{X} .
$$

We have $\alpha_{1}^{X} \leq \alpha_{1}^{Y}$, which completes the proof.

\section{B.3 Proof of Proposition 3}

Suppose that $\succeq$ satisfies Pride-Based Dominance (Axiom 12). Then, we show that $\beta_{S}\left(\overline{\boldsymbol{u}}_{A}\right)=$ 0 for all $A \in \mathcal{A}$. Suppose that $\beta_{1}\left(\overline{\boldsymbol{u}}_{A}\right) \neq 0$ for some $A \in \mathcal{A}$, and that the conditions in Axiom 12 (Pride-Based Dominance) hold. Then, $V(A)>V(A \cup B)$ or $V(A)<V(A \cup B)$ occurs. Without loss of generality, assume $\Delta_{S}(A \cup B)<0$. By Axiom 12, we must have $A \sim A \cup B \Leftrightarrow V(A)=V(A \cup B)$. However, if $\beta_{S}\left(\overline{\boldsymbol{u}}_{A \cup B}\right)<0$, then $V(A)>V(A \cup B)$. This is a contradiction. Hence, $\beta_{S}\left(\overline{\boldsymbol{u}}_{A}\right)=0$ for all $A \in \mathcal{A}$.

Fix $\boldsymbol{p}, \boldsymbol{q} \in \Delta^{I}$ such that the doubleton $\{\boldsymbol{p}, \boldsymbol{q}\}$ is a socially conflicting menu. If $\{\boldsymbol{p}, \boldsymbol{q}\} \succeq$ $\{\boldsymbol{p}\}$, then $V(\{\boldsymbol{p}, \boldsymbol{q}\}) \geq V(\{\boldsymbol{p}\}) \Leftrightarrow V(\{\boldsymbol{p}, \boldsymbol{q}\})-V(\{\boldsymbol{q}\}) \geq 0$. Notice that $\Delta_{1}=u\left(p_{1}\right)-$ $\bar{u}_{1}(\{\boldsymbol{p}, \boldsymbol{q}\})<0$. Hence, $\{\boldsymbol{p}, \boldsymbol{q}\} \succeq\{\boldsymbol{p}\} \Leftrightarrow V(\{\boldsymbol{p}, \boldsymbol{q}\})-V(\{\boldsymbol{q}\}) \geq 0 \Leftrightarrow \beta_{1}\left(\overline{\boldsymbol{u}}_{\{\boldsymbol{p}, \boldsymbol{q}\}}\right) \alpha_{1} \Delta_{1} \geq 0 \Leftrightarrow$ $-1<\beta_{1}\left(\overline{\boldsymbol{u}}_{\{\boldsymbol{p}, \boldsymbol{q}\}}\right) \leq 0$.

By Axiom 6 (Singleton Independence), we can show that this holds for any doubleton. Now, consider an arbitrary menu $A \in \mathcal{A}$ with $\boldsymbol{p}, \boldsymbol{q}$. If $\boldsymbol{p}$ is the maximizer in $A$, then $-1<$ $\beta_{1}\left(\overline{\boldsymbol{u}}_{A}\right) \leq 0$ holds. Suppose not. Then, $\boldsymbol{p}^{\prime}$ is the maximizer in $A$. Since $\beta_{S}\left(\overline{\boldsymbol{u}}_{A}\right)=0$, by Axiom 14 (Pride), there exists $\boldsymbol{q}^{\prime} \in A$ such that the doubleton $\left\{\boldsymbol{p}^{\prime}, \boldsymbol{q}^{\prime}\right\}$ is a socially conflicting menu. Moreover, by Axiom 13 (Self-interest), $\gamma_{1}=1$ holds (Corollary 3). $\Delta_{1}=u\left(p_{1}\right)-\bar{u}_{1}(A)<0$ holds for all $A \in \mathcal{A}$. Thus, $-1<\beta_{1}\left(\overline{\boldsymbol{u}}_{A}\right) \leq 0$ holds. Hence, $-1<\beta_{1}\left(u(\Delta)^{2}\right) \leq 0$. The necesity part is easily verified. We omit it.

\section{B.4 Proof of Proposition 4}

In Proposition 3 , we have $\beta_{S}\left(\overline{\boldsymbol{u}}_{A}\right)=0$ for all $A \in \mathcal{A}$. By Corollary $3, \gamma_{1}=1$.

the Sufficiency Part. We show the sufficiency part. By Proposition 3, for each $j \in\{X, Y\}$, $\succeq^{j}$ exhibits Pride if and only if $-1<\beta_{1}\left(u(\Delta)^{2}\right) \leq 0$. Take $\boldsymbol{p}, \boldsymbol{q} \in \Delta^{I}$ such that $\{\boldsymbol{p}, \boldsymbol{q}\}$ is a socially conflicting menu. And, take $r \in \Delta$. Suppose that $\succeq^{X}$ is more pride-seeking than $\succeq^{Y}$. Then, $\{\boldsymbol{p}, \boldsymbol{q}\} \succeq^{Y}\{\boldsymbol{p}\} \Rightarrow\{\boldsymbol{p}, \boldsymbol{q}\} \succeq^{X}\{\boldsymbol{p}\}$. Then, $V^{Y}(\{\boldsymbol{p}, \boldsymbol{q}\}) \geq V^{Y}(\{\boldsymbol{p}\})=U^{Y}(\{\boldsymbol{p}\})$. We also have $V^{X}(\{\boldsymbol{p}, \boldsymbol{q}\}) \geq V^{X}(\{\boldsymbol{p}\})=U^{X}(\{\boldsymbol{p}\})$. Since Mr. $X$ and Mr. $Y$ have the same $u, \boldsymbol{\alpha}$, and $\boldsymbol{\gamma}$, we obtain $V^{Y}(\{\boldsymbol{p}\})=U^{Y}(\{\boldsymbol{p}\})=V^{X}(\{\boldsymbol{p}\})=U^{X}(\{\boldsymbol{p}\})=\bar{U}$. Then, $V^{Y}(\{\boldsymbol{p}, \boldsymbol{q}\})-\bar{U} \leq V^{X}(\{\boldsymbol{p}, \boldsymbol{q}\})-\bar{U} \Leftrightarrow V^{Y}(\{\boldsymbol{p}, \boldsymbol{q}\}) \leq V^{X}(\{\boldsymbol{p}, \boldsymbol{q}\})$. Since $\Delta_{1}(\{\boldsymbol{p}, \boldsymbol{q}\}) \leq 0$ and $\alpha_{1}>0$, we obtan $-1<\beta^{X}\left(\overline{\boldsymbol{u}}_{\{\boldsymbol{p}, \boldsymbol{q}\}}\right) \leq \beta^{Y}\left(\overline{\boldsymbol{u}}_{\{\boldsymbol{p}, \boldsymbol{q}\}}\right) \leq 0$. By using Corollary 3 , we can show that this holds for any $A \in \mathcal{A}$. 
the Necessity Part. We show the necessity part. Assume that $-1<\beta^{X}\left(\overline{\boldsymbol{u}}_{A}\right) \leq \beta^{Y}\left(\overline{\boldsymbol{u}}_{A}\right) \leq 0$. In the same way, take $\boldsymbol{p}, \boldsymbol{q} \in \Delta^{I}$ such that $\{\boldsymbol{p}, \boldsymbol{q}\}$ is a socially conflicting menu. And, take $r \in \Delta$. By $\beta_{S}\left(\overline{\boldsymbol{u}}_{A}\right)=0$ for all $A \in \mathcal{A}$. By Corollary $3, \gamma_{1}=1$. We have $V^{X}(\{\boldsymbol{p}, \boldsymbol{q}\}) \geq$ $V^{X}(\{\boldsymbol{p}\})=U^{X}(\{\boldsymbol{p}\})$. In the same way, we obtain $V^{Y}(\{\boldsymbol{p}, \boldsymbol{q}\}) \leq V^{X}(\{\boldsymbol{p}, \boldsymbol{q}\})$. By the assumption of $-1<\beta^{X}\left(\overline{\boldsymbol{u}}_{A}\right) \leq \beta^{Y}\left(\overline{\boldsymbol{u}}_{A}\right) \leq 0$, we obtain the following property: $\succeq^{X}$ exhibits more pride-seeking than $\succeq^{Y}$.

\section{B.5 Proof of Proposition 5}

The proof of Proposition 5 is similar to that of Proposition 3. First, in Proposition 3, we have $\beta_{S}\left(\overline{\boldsymbol{u}}_{A}\right)=0$ for all $A \in \mathcal{A}$.

Second, in the similar way of Corollary 3 , we can show that $\gamma_{1}=0$.

Take $\boldsymbol{p}, \boldsymbol{q} \in \Delta^{I}$ such that the doubleton $\{\boldsymbol{p}, \boldsymbol{q}\}$ is a selfishly conflicting menu. If $\{\boldsymbol{p}, \boldsymbol{q}\} \succeq$ $\{\boldsymbol{p}\}$, then $V(\{\boldsymbol{p}, \boldsymbol{q}\}) \geq V(\{\boldsymbol{p}\}) \Leftrightarrow V(\{\boldsymbol{p}, \boldsymbol{q}\})-V(\{\boldsymbol{q}\}) \geq 0$. Notice that $\Delta_{1}=u\left(p_{1}\right)-$ $\bar{u}_{1}(\{\boldsymbol{p}, \boldsymbol{q}\}) \geq 0$. Hence, $\{\boldsymbol{p}, \boldsymbol{q}\} \succeq\{\boldsymbol{p}\} \Leftrightarrow V(\{\boldsymbol{p}, \boldsymbol{q}\})-V(\{\boldsymbol{q}\}) \geq 0 \Leftrightarrow \beta_{1}\left(\overline{\boldsymbol{u}}_{\{\boldsymbol{p}, \boldsymbol{q}\}}\right) \alpha_{1} \Delta_{1} \geq 0 \Leftrightarrow$ $0 \leq \beta_{1}\left(\overline{\boldsymbol{u}}_{\{\boldsymbol{p}, \boldsymbol{q}\}}\right) \leq+\infty$. In the same way as Proposition 3 , we can extend that this holds for any $A \in \mathcal{A}$.

\section{B.6 Proof of Proposition 6}

The proof of Proposition 6 is similar to Proposition 4. In Proposition 3, we have $\beta_{S}\left(\overline{\boldsymbol{u}}_{A}\right)=0$ for all $A \in \mathcal{A}$. Moreover, we have $\gamma_{1}=0$.

the Sufficiency Part. We show the sufficiency part. By Proposition 5, for each $j \in\{X, Y\}$, $\succeq^{j}$ exhibits Temptation if and only if $0 \leq \beta_{1}\left(u(\Delta)^{2}\right)<+\infty$. Take $\boldsymbol{p}, \boldsymbol{q} \in \Delta^{I}$ such that $\{\boldsymbol{p}, \boldsymbol{q}\}$ is a selfishly conflicting menu. And, take $r \in \Delta$. Suppose that $\succeq^{X}$ is more temptation-driven than $\succeq^{Y}$. Then, $\{\boldsymbol{p}, \boldsymbol{q}\} \succeq^{Y}\{\boldsymbol{p}\} \Rightarrow\{\boldsymbol{p}, \boldsymbol{q}\} \succeq^{X}\{\boldsymbol{p}\}$. Then, $V^{Y}(\{\boldsymbol{p}, \boldsymbol{q}\}) \geq V^{Y}(\{\boldsymbol{p}\})=U^{Y}(\{\boldsymbol{p}\})$. We also have $V^{X}(\{\boldsymbol{p}, \boldsymbol{q}\}) \geq V^{X}(\{\boldsymbol{p}\})=U^{X}(\{\boldsymbol{p}\})$. Since Mr. $X$ and Mr. $Y$ have the same $u, \boldsymbol{\alpha}$, and $\boldsymbol{\gamma}$, we obtain $V^{Y}(\{\boldsymbol{p}\})=U^{Y}(\{\boldsymbol{p}\})=V^{X}(\{\boldsymbol{p}\})=U^{X}(\{\boldsymbol{p}\})=\bar{U}$. Then, $V^{Y}(\{\boldsymbol{p}, \boldsymbol{q}\})-\bar{U} \leq V^{X}(\{\boldsymbol{p}, \boldsymbol{q}\})-\bar{U} \Leftrightarrow V^{Y}(\{\boldsymbol{p}, \boldsymbol{q}\}) \leq V^{X}(\{\boldsymbol{p}, \boldsymbol{q}\})$. Since $\Delta_{1}(\{\boldsymbol{p}, \boldsymbol{q}\}) \geq 0$ and $\alpha_{1}>0$, we obtan $0 \leq \beta^{Y}\left(\overline{\boldsymbol{u}}_{\{\boldsymbol{p}, \boldsymbol{q}\}}\right) \leq \beta^{X}\left(\overline{\boldsymbol{u}}_{\{\boldsymbol{p}, \boldsymbol{q}\}}\right)<+\infty$. By using $\gamma_{1}=0$, we can show that this holds for any $A \in \mathcal{A}$.

the Necessity Part. We show the necessity part. Assume that $0 \leq \beta^{Y}\left(\overline{\boldsymbol{u}}_{A}\right) \leq \beta^{X}\left(\overline{\boldsymbol{u}}_{A}\right)<$ $+\infty$. In the same way, take $\boldsymbol{p}, \boldsymbol{q} \in \Delta^{I}$ such that $\{\boldsymbol{p}, \boldsymbol{q}\}$ is a selfishly conflicting menu. And, take $r \in \Delta$. By $\beta_{S}\left(\overline{\boldsymbol{u}}_{A}\right)=0$ for all $A \in \mathcal{A}$. Furthermore, we have $\gamma_{1}=0$. We have $V^{X}(\{\boldsymbol{p}, \boldsymbol{q}\}) \geq V^{X}(\{\boldsymbol{p}\})=U^{X}(\{\boldsymbol{p}\})$. In the same way, we obtain $V^{Y}(\{\boldsymbol{p}, \boldsymbol{q}\}) \geq V^{X}(\{\boldsymbol{p}, \boldsymbol{q}\})$. By the assumption of $0 \leq \beta^{Y}\left(\overline{\boldsymbol{u}}_{A}\right) \leq \beta^{X}\left(\overline{\boldsymbol{u}}_{A}\right)<+\infty$, we obtain the following property: $\succeq^{X}$ exhibits more temptation-driven than $\succeq^{Y}$. 


\section{B.7 Proof of Proposition 7}

First, we show that $\beta_{1}\left(\overline{\boldsymbol{u}}_{A}\right)=0$ for all $A \in \mathcal{A}$. Suppose that $\beta_{1}\left(\overline{\boldsymbol{u}}_{A}\right) \neq 0$ for some $A \in \mathcal{A}$ by the way of contradiction. And, suppose that the conditions in Axiom 17 (Shame-Based Dominance) holds. Then, by the social emotions of pride or temptation, $V(A)>V(A \cup B)$ or $V(A)<V(A \cup B)$ occurs. Without loss of generality, assume $\Delta_{1}(A \cup B)<0$. By Axiom 17, we must have $A \sim A \cup B \Leftrightarrow V(A)=V(A \cup B)$. However, if $\beta_{1}\left(\overline{\boldsymbol{u}}_{A \cup B}\right)<0$, then $V(A)<V(A \cup B)$. This is a contradiction. Hence, $\beta_{1}\left(\overline{\boldsymbol{u}}_{A}\right)=0$ for all $A \in \mathcal{A}$.

By Corollary 4 , we have $\gamma_{S}=1$.

Take $\boldsymbol{p}, \boldsymbol{q} \in \Delta^{I}$ such that the doubleton $\{\boldsymbol{p}, \boldsymbol{q}\}$ is a shame-driven menu. If $\{\boldsymbol{p}\} \succeq\{\boldsymbol{p}, \boldsymbol{q}\}$, then $V(\{\boldsymbol{p}\}) \geq V(\{\boldsymbol{p}, \boldsymbol{q}\}) \Leftrightarrow V(\{\boldsymbol{p}\})-V(\{\boldsymbol{p}, \boldsymbol{q}\}) \geq 0$. Notice that $\Delta_{S}=u\left(p_{S}\right)-\bar{u}_{S}(\{\boldsymbol{p}, \boldsymbol{q}\}) \leq$ 0. Hence, $\{\boldsymbol{p}\} \succeq\{\boldsymbol{p}, \boldsymbol{q}\} \Leftrightarrow V(\{\boldsymbol{q}\})-V(\{\boldsymbol{p}, \boldsymbol{q}\}) \geq 0 \Leftrightarrow \beta_{S}\left(\overline{\boldsymbol{u}}_{\{\boldsymbol{p}, \boldsymbol{q}\}}\right) \alpha_{1} \Delta_{1} \leq 0 \Leftrightarrow-\infty \leq$ $\beta_{S}\left(\overline{\boldsymbol{u}}_{\{\boldsymbol{p}, \boldsymbol{q}\}}\right) \leq 0$. In the same way as Proposition 3, we can extend that this holds for any $A \in \mathcal{A}$.

\section{B.8 Proof of Proposition 8}

The proof of Proposition 6 is similar to Proposition 4. In Proposition 5, we have $\beta_{1}\left(\overline{\boldsymbol{u}}_{A}\right)=0$ for all $A \in \mathcal{A}$. Moreover, we have $\gamma_{1}=0$.

the Sufficiency Part. We show the sufficiency part. By Proposition 7, for each $j \in\{X, Y\}$, $\succeq^{j}$ exhibits Shame if and only if $-\infty \leq \beta_{S}\left(u(\Delta)^{2}\right) \leq 0$. Take $\boldsymbol{p}, \boldsymbol{q} \in \Delta^{I}$ such that $\{\boldsymbol{p}, \boldsymbol{q}\}$ is a shame-driven menu. And, take $l \in \Delta$. Suppose that $\succeq^{X}$ is more shame-averse than $\succeq^{Y}$. Then, $\{\boldsymbol{p}\} \succeq^{Y}\{\boldsymbol{p}, \boldsymbol{q}\} \Rightarrow\{\boldsymbol{p}\} \succeq^{X}\{\boldsymbol{p}, \boldsymbol{q}\}$. Then, $V^{Y}(\{\boldsymbol{p}\})=U^{Y}(\{\boldsymbol{p}\}) \geq V^{Y}(\{\boldsymbol{p}, \boldsymbol{q}\})$. We also have $V^{X}(\{\boldsymbol{p}\})=U^{X}(\{\boldsymbol{p}\}) \geq V^{X}(\{\boldsymbol{p}, \boldsymbol{q}\})$. Since Mr. $X$ and Mr. $Y$ have the same $u, \boldsymbol{\alpha}$, and $\boldsymbol{\gamma}$, we obtain $V^{Y}(\{\boldsymbol{p}\})=U^{Y}(\{\boldsymbol{p}\})=V^{X}(\{\boldsymbol{p}\})=U^{X}(\{\boldsymbol{p}\})=\bar{U}$. Then, $\bar{U}-V^{Y}(\{\boldsymbol{p}, \boldsymbol{q}\}) \leq \bar{U}-V^{X}(\{\boldsymbol{p}, \boldsymbol{q}\}) \Leftrightarrow V^{Y}(\{\boldsymbol{p}, \boldsymbol{q}\}) \leq V^{X}(\{\boldsymbol{p}, \boldsymbol{q}\})$. Since $\Delta_{S}(\{\boldsymbol{p}, \boldsymbol{q}\}) \leq 0$ and $\alpha_{1}>0$, we obtain $-\infty \leq \beta^{Y}\left(\overline{\boldsymbol{u}}_{\{\boldsymbol{p}, \boldsymbol{q}\}}\right) \leq \beta^{X}\left(\overline{\boldsymbol{u}}_{\{\boldsymbol{p}, \boldsymbol{q}\}}\right) \leq 0$. By using $\gamma_{1}=1$, we can show that this holds for any $A \in \mathcal{A}$.

the Necessity Part. We show the necessity part. Assume that $-\infty \leq \beta^{Y}\left(\overline{\boldsymbol{u}}_{A}\right) \leq \beta^{X}\left(\overline{\boldsymbol{u}}_{A}\right) \leq$ 0 . In the same way, take $\boldsymbol{p}, \boldsymbol{q} \in \Delta^{I}$ such that $\{\boldsymbol{p}, \boldsymbol{q}\}$ is a shame-driven menu. And, take $r \in \Delta$. We have $\beta_{1}\left(\overline{\boldsymbol{u}}_{A}\right)=0$ for all $A \in \mathcal{A}$. Furthermore, we have $\gamma_{1}=0$. We have $V^{X}(\{\boldsymbol{p}, \boldsymbol{q}\}) \leq V^{X}(\{\boldsymbol{p}\})=U^{X}(\{\boldsymbol{p}\})$. In the same way, we obtain $V^{Y}(\{\boldsymbol{p}, \boldsymbol{q}\}) \leq V^{X}(\{\boldsymbol{p}, \boldsymbol{q}\})$. By the assumption of $-\infty \leq \beta^{Y}\left(\overline{\boldsymbol{u}}_{A}\right) \leq \beta^{X}\left(\overline{\boldsymbol{u}}_{A}\right) \leq 0$, we obtain the following property: $\succeq^{X}$ exhibits more shame-averse than $\succeq^{Y}$. 


\section{B.9 Proof of Proposition 9}

Suppose that $\succeq$ is represented by a RDIC with a four-tuple $(u, \boldsymbol{\alpha}, \boldsymbol{\beta}, \boldsymbol{\gamma})$. By Corollary $4, \succeq$ satisfying Axiom 18 (Social-Interest Dominance) implies that $\gamma_{S}=1$.

We show that $\beta_{1}\left(\overline{\boldsymbol{u}}_{A}\right)=0$ for all $A \in \mathcal{A}$. Suppose that $\beta_{1}\left(\overline{\boldsymbol{u}}_{A}\right) \neq 0$ for some $A \in \mathcal{A}$ by the way of contradiction. And, suppose that the conditions in Axiom 17 (Shame-Based Dominance) holds. Then, by the social emotions of pride or temptation, $V(A)>V(A \cup B)$ or $V(A)<V(A \cup B)$ occurs. Without loss of generality, assume $\Delta_{1}(A \cup B)<0$. By Axiom 17, we must have $A \sim A \cup B \Leftrightarrow V(A)=V(A \cup B)$. However, if $\beta_{1}\left(\overline{\boldsymbol{u}}_{A \cup B}\right)<0$, then $V(A)<V(A \cup B)$. This is a contradiction. Hence, $\beta_{1}\left(\overline{\boldsymbol{u}}_{A}\right)=0$ for all $A \in \mathcal{A}$.

By Axiom 19 (Shame-Aversion), consider the menus $A, B \in \mathcal{A}$ with $A \succ_{S} A \cup B$. Let $\Delta_{S}(A):=\sum_{i \in S} \alpha_{i} u\left(p_{i}\right)-\bar{u}_{A}^{S}$ for each $A \in \mathcal{A}$ where $\boldsymbol{p}$ is a maximizer in $A$. Since $\gamma_{S}=1$, $\Delta_{S}(A) \leq 0$ for each $A \in \mathcal{A}$. Since $\beta_{1}\left(\overline{\boldsymbol{u}}_{A}\right)=0$ for all $A \in \mathcal{A}, A \succ A \cup B \Leftrightarrow V(A)>V(A \cup B)$ implies that $-\infty<\beta_{S}\left(\overline{\boldsymbol{u}}_{A \cup B}\right) \leq 0$.

Moreover, by the way of contradiction, suppose that $\beta_{S}\left(\overline{\boldsymbol{u}}_{A}\right)>0$. Since $\Delta_{S}(A)<0$, if $\boldsymbol{p}$ is a maximiser in $A \cup B$ with $\boldsymbol{p} \in A$, then $\Delta_{S}(A \cup B)<\Delta_{S}(A)<0 . \Delta_{S}(A \cup B)<\Delta_{S}(A)<0$ and $-\infty<\beta_{S}\left(\overline{\boldsymbol{u}}_{A \cup B}\right) \leq 0$ lead to $V(A \cup B) \leq V(A)$ beacues the positivity of the third term in the RDIC utility representation $V(A)$. This is a contradiction. Therefore, $-\infty<\beta_{S}\left(u(\Delta)^{2}\right) \leq 0$, $\beta_{1}\left(u(\Delta)^{2}\right)=0$, and $\gamma_{S}=1$ hold.

\section{B.10 Proof of Proposition 10}

The proof of Lemma 10 follows from Corollary 2 in Olszewski (2007). We show the sufficiency part in (i) and (ii). The necessity part is immediately shown. Take $A, B \in \mathcal{A}$ with $B \subset A$. Consider $j \in\{1, S\}$. Suppose that $A \succeq_{j}^{Y} B \Rightarrow A \succeq_{j}^{X} B$. By Step 2 in the proof of Theorem $1, \bar{u}_{j}^{Y}(A) \geq \bar{u}_{j}^{Y}(B) \Rightarrow \bar{u}_{j}^{X}(A) \geq \bar{u}_{j}^{X}(B)$. Hence, we have

$$
1 \geq \frac{\bar{u}_{j}^{Y}(A)-\bar{u}_{j}^{Y}(B)}{\bar{u}_{j}^{X}(A)-\bar{u}_{j}^{X}(B)} \geq 0 .
$$

Since both $X$ and $Y$ has the same self-utility function $u$, the maximum and the minimum of $j$ in each menu $A$ or $B$ are the same. Thus, the inequality implies $\gamma_{j}^{X} \geq \gamma_{j}^{Y}$.

\section{B.11 Proof of Proposition 11}

Take $\boldsymbol{p}, \boldsymbol{q} \in \Delta^{I}$ with (i) $\{\boldsymbol{q}\} \succ_{1}\{\boldsymbol{p}\}$, (ii) $\{\boldsymbol{p}\} \succ_{S}\{\boldsymbol{q}\}$, and (iii) $\{\boldsymbol{p}, \boldsymbol{q}\} \succ\{\boldsymbol{q}\}$. Fix the socially conflicting menu $\{\boldsymbol{p}, \boldsymbol{q}\}$.

Suppose that $\succeq$ is represented by a RDIC with a four-tuple $(u, \boldsymbol{\alpha}, \boldsymbol{\beta}, \boldsymbol{\gamma})$. Consider the 
menu $\{\boldsymbol{p}, \boldsymbol{q}\}$, and the utility of the menu is described as follows.

$$
\begin{aligned}
V(\{\boldsymbol{p}, \boldsymbol{q}\})=\max _{\boldsymbol{p} \in\{\boldsymbol{p}, \boldsymbol{q}\}}\left[\sum_{i \in I} \alpha_{i} u\left(p_{i}\right)\right. & +\beta_{1}\left(\overline{\boldsymbol{u}}_{A}\right)\left(\alpha_{1}\left(u\left(p_{1}\right)-\bar{u}_{1}(\{\boldsymbol{p}, \boldsymbol{q}\})\right)\right) \\
& \left.-\beta_{S}\left(\overline{\boldsymbol{u}}_{\{\boldsymbol{p}, \boldsymbol{q}\}}\right)\left(\sum_{i \in S} \alpha_{i} u\left(p_{i}\right)-\bar{u}_{S}(\{\boldsymbol{p}, \boldsymbol{q}\})\right)\right] .
\end{aligned}
$$

Let $\sum_{i \in I} \alpha_{i} u\left(p_{i}\right), \beta_{1}\left(\overline{\boldsymbol{u}}_{\{\boldsymbol{p}, \boldsymbol{q}\}}\right)\left(\alpha_{1}\left(u\left(p_{1}\right)-\bar{u}_{1}(\{\boldsymbol{p}, \boldsymbol{q}\})\right)\right),-\beta_{S}\left(\overline{\boldsymbol{u}}_{\{\boldsymbol{p}, \boldsymbol{q}\}}\right)\left(\sum_{i \in S} \alpha_{i} u\left(p_{i}\right)-\bar{u}_{S}(\{\boldsymbol{p}, \boldsymbol{q}\})\right)$ be the first term, the second term, and the third term, respectively. For each menu $A \in \mathcal{A}$, let $\Delta_{1}(A):=\alpha_{1}\left(u\left(p_{1}\right)-\bar{u}_{1}(A)\right)$ and $\left.\Delta_{S}(A):=\sum_{i \in S} \alpha_{i} u\left(p_{i}\right)-\bar{u}_{S}(A)\right)$ where $\boldsymbol{p}$ is a maximizer in $A$.

\section{Proof of (i) and (ii)}

The Sufficiency Part. First, we show the sufficiency part. Take a selfish option $\boldsymbol{r}$ for the socially conflicting menu $\{\boldsymbol{p}, \boldsymbol{q}\}$. Suppose that $\succeq$ exhibits more pride-seeking preferences. Then, we have $-1<\beta_{1}\left(\overline{\boldsymbol{u}}_{\{\boldsymbol{p}, \boldsymbol{q}\}}\right) \leq 0$. We need to show $-1<\beta_{1}\left(\overline{\boldsymbol{u}}_{\{\boldsymbol{p}, \boldsymbol{q}, \boldsymbol{r}\}}\right) \leq \beta_{1}\left(\overline{\boldsymbol{u}}_{\{\boldsymbol{p}, \boldsymbol{q}\}}\right) \leq 0$.

By pride-seeking preferences, $\{\boldsymbol{p}\} \prec\{\boldsymbol{p}, \boldsymbol{q}\} \Leftrightarrow V(\{\boldsymbol{p}\})<V(\{\boldsymbol{p}, \boldsymbol{q}\})$. We prove it. Since $\gamma_{j} \in[0,1]$ for each $j \in\{1, S\}$, we have $\Delta_{1}(\{\boldsymbol{p}, \boldsymbol{q}\}) \leq 0$ and $\Delta_{S}(\{\boldsymbol{p}, \boldsymbol{q}\}) \geq 0$. The condition (iii) $\{\boldsymbol{p}, \boldsymbol{q}\} \succ\{\boldsymbol{q}\}$ implies that $\boldsymbol{p}$ is chosen from $\{\boldsymbol{p}, \boldsymbol{q}\}$. Without loss of generality, $\boldsymbol{p}$ is a maximizer in $\{\boldsymbol{p}, \boldsymbol{q}\}$. Consider the second term in RDIC of $V(\{\boldsymbol{p}, \boldsymbol{q}\})$. By pride-seeking preferences, $-1<\beta_{1}(\overline{\boldsymbol{u}}(\{\boldsymbol{p}, \boldsymbol{q}\})) \leq 0$. And, since $\Delta_{1}(\{\boldsymbol{p}, \boldsymbol{q}\}) \leq 0$, the second term is nonnegative. If $\beta_{S}(\overline{\boldsymbol{u}}(\{\boldsymbol{p}, \boldsymbol{q}\}))>0$, then the third term is negative because $\Delta_{S}(\{\boldsymbol{p}, \boldsymbol{q}\}) \geq 0$. We must obtain (the second term) $\geq$ (the third term). If not, $V(\{\boldsymbol{p}, \boldsymbol{q}\})<V(\{\boldsymbol{p}\})$, which is not consistent with pride-seeking preferneces. On the other hand, if $\beta_{S}(\overline{\boldsymbol{u}}(\{\boldsymbol{p}, \boldsymbol{q}\})) \leq 0$, then the third term is non-negative. Hence, $V(\{\boldsymbol{p}\}) \leq V(\{\boldsymbol{p}, \boldsymbol{q}\}) \Leftrightarrow\{\boldsymbol{p}\} \preceq\{\boldsymbol{p}, \boldsymbol{q}\}$.

Consider the menu $\{\boldsymbol{p}, \boldsymbol{r}\}$ such that $\boldsymbol{r}$ is selfish than $\boldsymbol{p}$. By pride-seeking preferences, without loss of generality, assume that $\boldsymbol{p}$ is chosen from $\{\boldsymbol{p}, \boldsymbol{r}\}$. By the RDIC representation, $\Delta_{1}(\{\boldsymbol{p}, \boldsymbol{r}\}) \leq 0$. In the same way, $\Delta_{S}(\{\boldsymbol{p}, \boldsymbol{r}\}) \geq 0$. By the way of contradiction, suppose that $\beta_{1}\left(\overline{\boldsymbol{u}}_{\{\boldsymbol{p}, \boldsymbol{r}\}}\right) \geq 0$. Suppose also $\beta_{S}\left(\overline{\boldsymbol{u}}_{\{\boldsymbol{p}, \boldsymbol{r}\}}\right) \geq 0$. Thus, we have the following. Both the second term and the third term are non-positive in the RDIC representation of $V(\{\boldsymbol{p}, \boldsymbol{r}\})$. This implies that $V(\{\boldsymbol{p}\})>V(\{\boldsymbol{p}, \boldsymbol{r}\})$. This is a contradiction. On the other hand, supoose $-\infty<\beta_{S}\left(\overline{\boldsymbol{u}}_{\{\boldsymbol{p}, \boldsymbol{r}\}}\right) \leq 0$. Then, by the definition of socially conflicting of doubletons, |(the second term $)|<|$ (the third term) $\mid$ because $\boldsymbol{p}$ is chosen from $\{\boldsymbol{p}, \boldsymbol{r}\}$ at the ex-post stage. We obtain $V(\{\boldsymbol{p}\})>V(\{\boldsymbol{p}, \boldsymbol{r}\})$. This is a contradiction. Hence, we obtain $\beta_{1}\left(\overline{\boldsymbol{u}}_{\{\boldsymbol{p}, \boldsymbol{r}\}}\right) \leq 0$.

Now, consider the menu $\{\boldsymbol{p}, \boldsymbol{q}, \boldsymbol{r}\}$. By pride-seeking preferences, under the conditions, $\{\boldsymbol{p}\} \preceq\{\boldsymbol{p}, \boldsymbol{q}\} \Rightarrow\{\boldsymbol{p}\} \preceq\{\boldsymbol{p}, \boldsymbol{r}\}$. By socially conflicting menus, we have $\beta_{1}\left(\overline{\boldsymbol{u}}_{\{\boldsymbol{p}, \boldsymbol{q}\}}\right) \leq 0$, as shown in the above.

We need to show that $\beta_{1}\left(\overline{\boldsymbol{u}}_{\{\boldsymbol{p}, \boldsymbol{q}, \boldsymbol{r}\}}\right) \leq \beta_{1}\left(\overline{\boldsymbol{u}}_{\{\boldsymbol{p}, \boldsymbol{q}\}}\right)$. Consider the menu $\{\boldsymbol{p}, \boldsymbol{r}\}$. First, consider the case of the reference points of the two menus $\overline{\boldsymbol{u}}_{\{\boldsymbol{p}, \boldsymbol{q}, \boldsymbol{r}\}}$ and $\overline{\boldsymbol{u}}_{\{\boldsymbol{p}, \boldsymbol{r}\}}$ with $\overline{\boldsymbol{u}}_{\{\boldsymbol{p}, \boldsymbol{q}, \boldsymbol{r}\}}=\overline{\boldsymbol{u}}_{\{\boldsymbol{p}, \boldsymbol{r}\}}$. By 
the definition of $\boldsymbol{\beta}$, we obtain $\boldsymbol{\beta}\left(\overline{\boldsymbol{u}}_{\{\boldsymbol{p}, \boldsymbol{q}, \boldsymbol{r}\}}\right)=\boldsymbol{\beta}\left(\overline{\boldsymbol{u}}_{\{\boldsymbol{p}, \boldsymbol{r}\}}\right)$. As shown in the above, $\beta_{1}\left(\overline{\boldsymbol{u}}_{\{\boldsymbol{p}, \boldsymbol{r}\}}\right) \leq$ 0 and $\beta_{1}\left(\overline{\boldsymbol{u}}_{\{\boldsymbol{p}, \boldsymbol{q}\}}\right) \leq 0$. By pride-seeking preferences, $V(\{\boldsymbol{p}, \boldsymbol{r}\}) \leq V(\{\boldsymbol{p}, \boldsymbol{q}\})$ implies that $\beta_{1}\left(\overline{\boldsymbol{u}}_{\{\boldsymbol{p}, \boldsymbol{r}\}}\right) \leq \beta_{1}\left(\overline{\boldsymbol{u}}_{\{\boldsymbol{p}, \boldsymbol{q}\}}\right)$. Hence, $\beta_{1}\left(\overline{\boldsymbol{u}}_{\{\boldsymbol{p}, \boldsymbol{r}\}}\right)=\beta_{1}\left(\overline{\boldsymbol{u}}_{\{\boldsymbol{p}, \boldsymbol{q}, \boldsymbol{r}\}}\right) \leq \beta_{1}\left(\overline{\boldsymbol{u}}_{\{\boldsymbol{p}, \boldsymbol{q}\}}\right)$.

Next, consider the case of $\overline{\boldsymbol{u}}_{\{\boldsymbol{p}, \boldsymbol{q}, \boldsymbol{r}\}} \neq \overline{\boldsymbol{u}}_{\{\boldsymbol{p}, \boldsymbol{r}\}}$, and $\{\boldsymbol{p}\} \preceq\{\boldsymbol{p}, \boldsymbol{r}\}$. In this case, $\Delta_{1}(\{\boldsymbol{p}, \boldsymbol{q}, \boldsymbol{r}\}) \leq$ $\Delta_{1}(\{\boldsymbol{p}, \boldsymbol{q}\})$, and $\Delta_{S}(\{\boldsymbol{p}, \boldsymbol{q}, \boldsymbol{r}\}) \leq \Delta_{S}(\{\boldsymbol{p}, \boldsymbol{q}\}) . V(\{\boldsymbol{p}, \boldsymbol{q}\}) \geq V(\{\boldsymbol{p}, \boldsymbol{r}\})$ implies that $\beta_{1}(\cdot)$ must be $-1<\beta_{1}\left(\overline{\boldsymbol{u}}_{\{\boldsymbol{p}, \boldsymbol{q}, \boldsymbol{r}\}}\right) \leq \beta_{1}\left(\overline{\boldsymbol{u}}_{\{\boldsymbol{p}, \boldsymbol{q}\}}\right) \leq 0$. Hence, in the case that $\succeq$ exhibits pride-seeking preferences, $\beta_{1}$ is decreasing in the first argument.

The Necessity Part. Next, we show the necessity part. Take a selfish option $\mathbf{r}$ for the menu $\{\boldsymbol{p}, \boldsymbol{q}\}$. Suppose that $-1<\beta_{1}\left(\overline{\boldsymbol{u}}_{\{\boldsymbol{p}, \boldsymbol{q}, \boldsymbol{r}\}}\right) \leq \beta_{1}\left(\overline{\mathbf{u}}_{\{\boldsymbol{p}, \boldsymbol{q}\}}\right) \leq 0$. $\beta_{1}$ is decreasing in the first argument on $(-1,0)$. $\{\boldsymbol{p}\} \preceq\{\boldsymbol{p}, \boldsymbol{q}\}$ holds with the three conditions implies that $\beta_{1}\left(\overline{\boldsymbol{u}}_{\{\boldsymbol{p}, \boldsymbol{q}\}}\right) \leq$ 0 . Then, we have $\beta_{1}\left(\overline{\boldsymbol{u}}_{\{\boldsymbol{p}, \boldsymbol{q}, \boldsymbol{r}\}}\right) \leq \beta_{1}\left(\overline{\mathbf{u}}_{\{\boldsymbol{p}, \boldsymbol{q}\}}\right) \leq 0$.

First, consider the case of $\overline{\boldsymbol{u}}_{\{\boldsymbol{p}, \boldsymbol{q}, \boldsymbol{r}\}}=\overline{\boldsymbol{u}}_{\{\boldsymbol{p}, \boldsymbol{r}\}}$. By the definition of $\boldsymbol{\beta}$, we obtain $\boldsymbol{\beta}\left(\overline{\boldsymbol{u}}_{\{\boldsymbol{p}, \boldsymbol{q}, \boldsymbol{r}\}}\right)=$ $\boldsymbol{\beta}\left(\overline{\boldsymbol{u}}_{\{\boldsymbol{p}, \boldsymbol{r}\}}\right)$. Hence, $\{\boldsymbol{p}, \boldsymbol{q}, \boldsymbol{r}\} \sim_{1}\{\boldsymbol{p}, \boldsymbol{r}\}$ and $\{\boldsymbol{p}, \boldsymbol{q}, \boldsymbol{r}\} \sim_{S}\{\boldsymbol{p}, \boldsymbol{r}\}$. This imply that in the menu $\{\boldsymbol{p}, \boldsymbol{r}\}, \succeq$ exhibits pride, and that $\{\boldsymbol{p}\} \preceq\{\boldsymbol{p}, \boldsymbol{r}\}$.

Second, consider the case of $\overline{\boldsymbol{u}}_{\{\boldsymbol{p}, \boldsymbol{q}, \boldsymbol{r}\}} \neq \overline{\boldsymbol{u}}_{\{\boldsymbol{p}, \boldsymbol{r}\}}$. Suppose that , by the way of contradiction, $\{\boldsymbol{p}, \boldsymbol{r}\} \preceq\{\boldsymbol{r}\}$. This case says that $\succeq$ exhibits shame of acting selfishly. Then, $\Delta_{1}(\{\boldsymbol{p}, \boldsymbol{r}\})<0$ and $\Delta_{S}(\{\boldsymbol{p}, \boldsymbol{r}\})>0$. Suppose that $\beta_{1}(\{\boldsymbol{p}, \boldsymbol{r}\})>0$. Then, the second term is non-positive, and the third term is positive. By the definition of socially conflicting of doubletons, |(the second term $)|<|($ the third term $) \mid$ because $\boldsymbol{p}$ is chosen from $\{\boldsymbol{p}, \boldsymbol{r}\}$ at the ex-post stage. Thus, $V(\{\boldsymbol{p}, \boldsymbol{r}\})<V(\{\boldsymbol{r}\})$. This is a contradiction. Thus, we obtain $\{\boldsymbol{p}, \boldsymbol{r}\} \succ\{\boldsymbol{r}\}$. Hence, the three conditions are satisfied, and $-1<\beta_{1}\left(\overline{\boldsymbol{u}}_{\{\boldsymbol{p}, \boldsymbol{q}, \boldsymbol{r}\}}\right) \leq \beta_{1}\left(\overline{\mathbf{u}}_{\{\boldsymbol{p}, \boldsymbol{q}\}}\right) \leq 0$, so $\{\boldsymbol{p}\} \preceq\{\boldsymbol{p}, \boldsymbol{r}\}$.

In the same way, we can show the first part of Proposition 11. We omit it.

\section{B.12 Proof of Proposition 12}

Take $\boldsymbol{p}, \boldsymbol{q} \in \Delta^{I}$. Fix a menu $\{\boldsymbol{p}, \boldsymbol{q}\}$, and suppose the following. Take an arbitrary option $\boldsymbol{r} \in \Delta^{I}$. Suppose (i) $\{\boldsymbol{r}\} \succ_{1}\{\boldsymbol{p}\}$, (ii) $\{\boldsymbol{q}\} \succ_{S}\{\boldsymbol{p}\}$, and (iii) $\{\boldsymbol{p}, \boldsymbol{q}, \boldsymbol{r}\} \succ\{\boldsymbol{q}, \boldsymbol{r}\}$. Take an altruistic option $\boldsymbol{r}^{\prime}$ for the menu $\{\boldsymbol{p}, \boldsymbol{q}\}$.

First, we show the sufficiency part. Suppose that $\succeq$ exhibits more shame-averse preferences. We need to show $\left|\beta_{S}\left(\overline{\boldsymbol{u}}_{\left\{\boldsymbol{p}, \boldsymbol{q}, \boldsymbol{r}^{\prime}\right\}}\right)\right| \geq\left|\beta_{S}\left(\overline{\boldsymbol{u}}_{\{\boldsymbol{p}, \boldsymbol{q}\}}\right)\right|$; that is, $\beta_{S}$ is decreasing in the second argument on $(-\infty, 0)$.

Consider the menu $\{\boldsymbol{p}, \boldsymbol{q}\}$. By the three conditions and $\{\boldsymbol{p}\} \succeq\{\boldsymbol{p}, \boldsymbol{q}\},-\infty<\beta_{S}\left(\overline{\boldsymbol{u}}_{\{\boldsymbol{p}, \boldsymbol{q}\}}\right) \leq$ 0 . Suppose that $-1<\beta_{1}\left(\overline{\boldsymbol{u}}_{\{\boldsymbol{p}, \boldsymbol{q}\}}\right) \leq 0$. Since $\Delta_{1}(\{\boldsymbol{p}, \boldsymbol{q}\})>0$ and $\Delta_{S}(\{\boldsymbol{p}, \boldsymbol{q}\})<0$, the second term is negative, and the third term is negative. Hence, $V(\{\boldsymbol{p}\}) \geq V(\{\boldsymbol{p}, \boldsymbol{q}\})$. Furthermore, consider the case of $0<\beta_{1}\left(\overline{\boldsymbol{u}}_{\{\boldsymbol{p}, \boldsymbol{q}\}}\right)<+\infty$. Then, the second term is positive. By shameaverse preferences, $\{\boldsymbol{p}\} \succeq\{\boldsymbol{p}, \boldsymbol{q}\}$, which implies $\mid$ (the second term) $|\leq|$ (the third term) $\mid$. Hence, $V(\{\boldsymbol{p}\}) \geq V(\{\boldsymbol{p}, \boldsymbol{q}\})$. 
Consider the menu $\left\{\boldsymbol{p}, \boldsymbol{r}^{\prime}\right\}$. By shame-averse preferences, $\{\boldsymbol{p}\} \succeq\left\{\boldsymbol{p}, \boldsymbol{r}^{\prime}\right\}$. By the similar argument above, we have $-\infty<\beta_{S}\left(\overline{\boldsymbol{u}}_{\left\{\boldsymbol{p}, \boldsymbol{r}^{\prime}\right\}}\right) \leq 0$. We obtain $V(\{\boldsymbol{p}\}) \geq V\left(\left\{\boldsymbol{p}, \boldsymbol{r}^{\prime}\right\}\right)$.

Furthermore, consider the two menus $\left\{\boldsymbol{p}, \boldsymbol{q}, \boldsymbol{r}^{\prime}\right\}$ and $\left\{\boldsymbol{p}, \boldsymbol{r}^{\prime}\right\}$. First, without loss of generality, assume that $\overline{\boldsymbol{u}}_{\left\{\boldsymbol{p}, \boldsymbol{q}, \boldsymbol{r}^{\prime}\right\}}=\overline{\boldsymbol{u}}_{\left\{\boldsymbol{p}, \boldsymbol{r}^{\prime}\right\}}$. By the definition of $\boldsymbol{\beta}, \beta_{S}\left(\overline{\boldsymbol{u}}_{\left\{\boldsymbol{p}, \boldsymbol{q}, \boldsymbol{r}^{\prime}\right\}}\right)=\beta_{S}\left(\overline{\boldsymbol{u}}_{\left\{\boldsymbol{p}, \boldsymbol{r}^{\prime}\right\}}\right) \leq$ $\beta_{S}\left(\overline{\boldsymbol{u}}_{\{\boldsymbol{p}, \boldsymbol{q}\}}\right)$.

Second, consider the case of $\overline{\boldsymbol{u}}_{\left\{\boldsymbol{p}, \boldsymbol{q}, \boldsymbol{r}^{\prime}\right\}} \neq \overline{\boldsymbol{u}}_{\left\{\boldsymbol{p}, \boldsymbol{r}^{\prime}\right\}}$. Since $\succeq$ exhibits more shame-averse preferences, we have the following: $V(\{\boldsymbol{p}\}) \geq V(\{\boldsymbol{p}, \boldsymbol{q}\}) \Rightarrow V(\{\boldsymbol{p}\}) \geq V\left(\left\{\boldsymbol{p}, \boldsymbol{r}^{\prime}\right\}\right)$. Then, $V(\{\boldsymbol{p}, \boldsymbol{q}\}) \leq V\left(\left\{\boldsymbol{p}, \boldsymbol{r}^{\prime}\right\}\right)$. Moreover, $\Delta_{S}\left(\left\{\boldsymbol{p}, \boldsymbol{q}, \boldsymbol{r}^{\prime}\right\}\right) \leq \Delta_{S}(\{\boldsymbol{p}, \boldsymbol{q}\})<0$. $\beta_{S}$ is decreasing in the second argument, i.e., $\beta_{S}\left(\overline{\boldsymbol{u}}_{\left\{\boldsymbol{p}, \boldsymbol{q}, \boldsymbol{r}^{\prime}\right\}}\right) \leq \beta_{S}\left(\overline{\boldsymbol{u}}_{\{\boldsymbol{p}, \boldsymbol{q}\}}\right)$.

Next, we show the necessity part. Suppose $\left|\beta_{S}\left(\overline{\boldsymbol{u}}_{\left\{\boldsymbol{p}, \boldsymbol{q}, \boldsymbol{r}^{\prime}\right\}}\right)\right| \geq\left|\beta_{S}\left(\overline{\mathbf{u}}_{\{\boldsymbol{p}, \boldsymbol{q}\}}\right)\right|$; that is, $\beta_{S}$ is decreasing in the second argument on $(-\infty, 0)$. $\succeq$ is shame-averse, i.e., $\{\boldsymbol{p}\} \succeq\{\boldsymbol{p}, \boldsymbol{q}\}$. We need to show $\{\boldsymbol{p}\} \succeq\left\{\boldsymbol{p}, \boldsymbol{r}^{\prime}\right\}$. By the way of contradiction, suppose that $\{\boldsymbol{p}\} \prec\left\{\boldsymbol{p}, \boldsymbol{r}^{\prime}\right\}$. Suppose that, at the ex-post stage, $\boldsymbol{p}$ is chosen from $\left\{\boldsymbol{p}, \boldsymbol{r}^{\prime}\right\}$. Then, $\Delta_{1}\left(\left\{\boldsymbol{p}, \boldsymbol{r}^{\prime}\right\}\right)>0$ and $\Delta_{S}\left(\left\{\boldsymbol{p}, \boldsymbol{r}^{\prime}\right\}\right)<0 . \quad\{\boldsymbol{p}\} \prec\left\{\boldsymbol{p}, \boldsymbol{r}^{\prime}\right\}$ implies that $\succeq$ exhibits pride of acting altruistically. Then, $\beta_{1}\left(\overline{\boldsymbol{u}}_{\left\{\boldsymbol{p}, \boldsymbol{r}^{\prime}\right\}}\right) \leq 0$. Without loss of generality, suppose $\beta_{S}\left(\overline{\boldsymbol{u}}_{\left\{\boldsymbol{p}, \boldsymbol{r}^{\prime}\right\}}\right) \leq 0$. Then, the second term is negative, and the third term is negative, so $V(\{\boldsymbol{p}\})>V\left(\left\{\boldsymbol{p}, \boldsymbol{r}^{\prime}\right\}\right)$. This is a contradiction.

\section{Proofs of Corollaries}

\section{C.1 Proof of Corollary 1}

Suppose that $\succeq$ is represented by a RDIC with a four-tuple $(u, \boldsymbol{\alpha}, \boldsymbol{\beta}, \boldsymbol{\gamma})$.

First, we show the sufficiency part. $\succeq$ satisfies Axiom 8, i.e., Strategic Rationality: $A \succeq$ $B \Rightarrow A \sim A \cup B$. Take arbitrary two menus, $A, B \in \mathcal{A}$ with $A \succeq B$. By Axiom 8, $A \sim A \cup B \Leftrightarrow V(A)=V(A \cup B)$. In the way of contradiction, suppose $\beta_{1}\left(\overline{\boldsymbol{u}}_{A \cup B}\right)>0$. Without loss of generality, $A \succ_{1} B$. Then, the second term in the RDIC is not equal to zero. In the case of $\beta_{1}\left(\overline{\boldsymbol{u}}_{A \cup B}\right)>0$, without $u_{1}\left(p_{1}\right)-\bar{u}_{A \cup B}^{1}=0, V(A)<V(A \cup B)$ or $V(A)>V(A \cup B)$ holds. This is a contradiciton. Hence, $\beta_{1}\left(\overline{\boldsymbol{u}}_{A}\right)=0$ holds for any $A \in \mathcal{A}$. We can show that $\beta_{S}\left(\overline{\boldsymbol{u}}_{A}\right)=0$ in the same way. Thus, for any $A \in \mathcal{A}, \beta_{1}\left(\overline{\boldsymbol{u}}_{A}\right)=\beta_{S}\left(\overline{\boldsymbol{u}}_{A}\right)=0$.

Second, we show the necessity part. Suppose that for any $A \in \mathcal{A}, \beta_{1}\left(\overline{\boldsymbol{u}}_{A}\right)=\beta_{S}\left(\overline{\boldsymbol{u}}_{A}\right)=0$. Then, for any $A \in \mathcal{A}$,

$$
V(A)=\max _{\boldsymbol{p} \in A} \alpha_{1} u\left(p_{1}\right)+\sum_{i \in S} \alpha_{i} u\left(p_{i}\right) .
$$

Take arbitrary two menus $A, B \in \mathcal{A}$ with $A \succeq B$. Then, $V(A) \geq V(B)$, which says that for any $\boldsymbol{q} \in B$, there exists $\boldsymbol{p} \in A$ such that $\{\boldsymbol{p}\} \succeq\{\boldsymbol{q}\}$. This implies that $V(A)=V(A \cup B)$. Thus, we obtain $A \succeq B \Rightarrow A \sim A \cup B$. 


\section{C.2 Proof of Corollary 2}

Suppose that $\succeq$ is represented by a RDIC with a four-tuple $(u, \boldsymbol{\alpha}, \boldsymbol{\beta}, \boldsymbol{\gamma})$.

We show the sufficiency part. Suppose that $\succeq$ satisfies Axioms 8 (Strategic Rationality), 9 (Monotonicity w.r.t. Equal Allocations), 10 (Comonotonic Independence), and 11 (Inequality Aversion). By Axiom $8, \beta_{1}\left(\overline{\boldsymbol{u}}_{A}\right)=\beta_{S}\left(\overline{\boldsymbol{u}}_{A}\right)=0$, for each $A \in \mathcal{A}$ (Corollary 1$)$.

In the same way as the step 3 in Theorem 1 , we consider the utility space of allocations. For any $\boldsymbol{p} \in \Delta^{I}$, define

$$
\boldsymbol{u}(\boldsymbol{p}):=\left\{\left(\left(u\left(p_{1}\right), \cdots, u\left(p_{n}\right)\right) \in \mathbb{R}^{I} \mid \boldsymbol{p} \in \Delta^{I}\right\} .\right.
$$

We consider a binary relation $\succsim^{*}$ on $\mathbb{R}^{I}$, and then verify that $\succsim^{*}$ on $\mathbb{R}^{I}$ satisfies the axioms in Corollary 2. This is straightforward, so we omit it.

The following step follows from Lemma 1 in Saito (2013). Let $U: \mathbb{R}^{I} \rightarrow \mathbb{R}$ that represents $\succsim^{*}$. We can show that (i) $U$ is mixture-linear with respect to comonotonic allocations, (ii) $U$ is homothetic, and that (iii) $U$ is unique up to positive affine transformation.

By (iii), we can normalize $U(1, \cdots, 1)=1$, and $U(0, \cdots, 0)=0$. Let $\alpha_{\text {envy }}^{i}:=-U\left(1,(0)_{-i}\right)$. By Axiom 11 (Inequality Aversion), take $p, p^{\prime} \in \Delta$ with $p^{\prime} \succ^{i} p$. Define

$$
\left(u\left(p_{i}^{\prime}\right)-u\left(p_{1}\right)\right) U\left(1,(0)_{i \in S}\right):=-\alpha_{\text {envy }}^{i} \max \left\{u\left(p_{i}^{\prime}\right)-u\left(p_{1}\right), 0\right\} .
$$

By (ii), we can prove it is well-defined. In the same way, the guilt part is shown. Take $p, p^{\prime} \in \Delta$ with $p \succ^{i} p^{\prime}$. Define

$$
\left(u\left(p_{1}\right)-u\left(p_{1}^{\prime}\right)\right) U\left(-1,(0)_{i \in S}\right):=-\alpha_{\text {guilt }}^{i} \max \left\{u\left(p_{1}\right)-u\left(p_{i}^{\prime}\right), 0\right\} .
$$

Hence, there exists a tuple $\left(u, \alpha_{1},\left(\alpha_{\text {envy }}^{i}, \alpha_{\text {guilt }}^{i}\right)_{i \in S}\right)$ where $u: \Delta \rightarrow \mathbb{R}$ is a non-constant function, $\alpha_{1}>0$, and for each $i \in S, \alpha_{\text {envy }}^{i} \geq 0, \alpha_{\text {guilt }}^{i} \geq 0$, such that $\succeq$ is represented by

$$
V(A)=\max _{\boldsymbol{p} \in A}\left[\alpha_{1} u\left(p_{1}\right)-\sum_{i \in S}\left(\alpha_{\text {envy }}^{i} \max \left\{u\left(p_{i}\right)-u\left(p_{1}\right), 0\right\}+\alpha_{\text {guilt }}^{i} \max \left\{u\left(p_{1}\right)-u\left(p_{i}\right), 0\right\}\right)\right] .
$$

\section{C.3 Proof of Corollary 3}

Suppose that $\succeq$ is represented by a RDIC with a four-tuple $(u, \boldsymbol{\alpha}, \boldsymbol{\beta}, \boldsymbol{\gamma})$.

We show the sufficiency part. Suppose that $\succeq_{1}$ exhibits Axiom 13 (Self-Interest Strategic Rationality). Remember that $\succeq_{1}$ is represented by $\bar{u}_{1}: \mathcal{A} \rightarrow \mathbb{R}$. Take arbitrary two menus $A, B \in \mathcal{A}$ with $A \succeq_{1} B$. By Axiom 13, $\bar{u}_{1}(A)=\bar{u}_{1}(A \cup B)$. By the definition of $\succeq_{1}$, $\bar{u}_{1}(A)=\bar{u}_{1}(A \cup B)$ implies that $\max _{\boldsymbol{p} \in A} u\left(p_{1}\right)=\max _{\boldsymbol{p} \in A \cup B} u\left(p_{1}\right)$. Hence, $\gamma_{1}=1$.

We show the necessity part. Suppose $\gamma_{1}=1$. Take arbitrary two menus $A, B \in \mathcal{A}$ with $A \succeq_{1} B$. Since $\gamma_{1}=1, \bar{u}_{1}(A) \geq \bar{u}_{1}(B) \Leftrightarrow \max _{\boldsymbol{p} \in A} u\left(p_{1}\right) \geq \max _{\boldsymbol{p} \in B} u\left(p_{1}\right)$. Thus, $\bar{u}_{1}(A)=\bar{u}_{1}(A \cup B)$ holds, because of $\max _{\boldsymbol{p} \in A} u\left(p_{1}\right)=\max _{\boldsymbol{p} \in A \cup B} u\left(p_{1}\right)$. Therefore, $\succeq_{1}$ satisfies Axiom 13. 


\section{C.4 Proof of Corollary 4}

The proof is similar to the proof of Corollary 3. We omit it.

\section{C.5 Proof of Corollary 5}

The proof follows from Lemma 1 in Segal and Sobel (2007). Define the correspondence $\Phi: \Sigma \rightrightarrows \Sigma$ by, for each $A \in \mathcal{A}$ and $\sigma^{*} \in \Sigma$,

$$
\Phi_{i}\left(\sigma^{*}\right):=\left\{\sigma_{i} \in A_{i} \mid\left\{\sigma_{i}\right\} \succeq\left\{\sigma_{i}^{\prime}\right\} \text { for all } \sigma_{i}^{\prime} \in A_{i}\right\}
$$

Since, given $\sigma^{*} \in \Sigma, \succeq_{i, \sigma^{*}}$ satisfies the axioms of the vNM-type expected utility theorem (EUT). $A_{i}$ is nonempty. The vNM-type EUT guarantees that $\Phi(\cdot)$ is convex. $A_{i}$ is compact. Hence, $\Phi(\cdot)$ satisfies the conditions in the Kakutani's fixed point theorem. Hence, a Nash equilibrium exists.

\section{C.6 Proof of Corollary 6}

The necessity part is easily shown. We omit it. We show the sufficiency part. Suppose that $\succeq$ satisfies Axioms 1, 2, 3, 6, 18, 17, and 20. By Axiom 20 (Shame-Based Independence), we slightly modifies the Step 4 in the sufficiency part of Theorem 1 . We replace the signed measure $\mu$ with $\mu_{S}\left(\bar{u}_{S}(A), \lambda\right)$, for all $A \in \mathcal{A}$ and $\lambda \in[0,1]$. Since we normalize the utility space, formally let $\mu_{S}:[0,1] \times[0,1] \rightarrow \mathbb{R}$. In the same way as the proof in Theorem 1 , we can obtain $\beta_{S}: u(\Delta) \rightarrow(-\infty, 1)$.

Moreover, we show that $\beta_{1}\left(\bar{u}_{S}(A)\right)=0$ for all $A \in \mathcal{A}$. Suppose that $\beta_{1}\left(\bar{u}_{S}(A)\right) \neq 0$ for some $A \in \mathcal{A}$, and that the conditions in Axiom 17 (Shame-Based Dominance) holds. Then, by the social emotions of pride or temptation, $V(A)>V(A \cup B)$ or $V(A)<V(A \cup B)$ occurs. Hence, $\beta_{1}\left(\bar{u}_{S}(A)\right)=0$ for all $A \in \mathcal{A}$.

By Corollary 4, $\succeq$ satisfying Axiom 18 (Social-interest Dominance) implies that $\gamma_{S}=1$. Hence, there exists a four-tuple $(u, \boldsymbol{\alpha}, \boldsymbol{\beta}, \boldsymbol{\gamma})$ with $\beta_{1}(\cdot)=0$, such that $\succeq$ is represented by

$$
V(A)=\max _{\boldsymbol{p} \in A}\left[\sum_{i \in I} \alpha_{i} u\left(p_{i}\right)-\beta_{S}\left(\max _{\boldsymbol{q} \in A} \sum_{i \in S} \alpha_{i} u\left(q_{i}\right)\right)\left(\sum_{i \in S} \alpha_{i} u\left(p_{i}\right)-\max _{\boldsymbol{q} \in A} \sum_{i \in S} \alpha_{i} u\left(q_{i}\right)\right)\right] .
$$

\section{C.7 Proof of Corollary 7}

The necessity part is easily shown. We omit it. We show the sufficiency part.

$\succeq$ satisfies Axioms 1, 2, 3, 6, 13, 12, and 21. The proof step is similar to the proof of Corollary 6. The difference is to replace the signed measure $\mu$ with $\mu_{1}\left(\bar{u}_{1}(A), \lambda\right)$, for all $A \in \mathcal{A}$ and $\lambda \in[0,1]$. By normalizing the utility space, formally let $\mu_{1}:[0,1] \times[0,1] \rightarrow \mathbb{R}$. 
By Corollary 3, $\succeq$ satisfying Axiom 13 (Self-Interest Dominance) implies that $\gamma_{1}=1$. Thus, we obtain a four-tuple $(u, \boldsymbol{\alpha}, \boldsymbol{\beta}, \boldsymbol{\gamma})$ with $\beta_{S}(u(\Delta))=0$, such that $\succeq$ is represented by

$$
V(A)=\max _{\boldsymbol{p} \in A}\left[\sum_{i \in I} \alpha_{i} u\left(p_{i}\right)+\beta_{1}\left(\max _{\boldsymbol{q} \in A} u\left(q_{1}\right)\right)\left(\alpha_{1}\left(u\left(p_{1}\right)-\max _{\boldsymbol{q} \in A} u\left(q_{1}\right)\right)\right)\right] .
$$

\section{C.8 Proof of Corollary 8}

The necessity part is easily shown. We omit it. We show the sufficiency part. By Corollary $3, \succeq$ satisfying Axiom 13 (Self-Interest Dominance) implies that $\gamma_{1}=1$. By Corollary 4, $\succeq$ satisfying Axiom 18 (Social-interest Dominance) implies that $\gamma_{S}=1$. By Corollary 6, $\beta_{S}: u(\Delta) \rightarrow(-\infty, 1)$. By Corollary $7, \beta_{1}: u(\Delta) \rightarrow(-1,+\infty)$. Thus, we obtain the desired utility representation: For any $A \in \mathcal{A}$, there exists a four-tuple $(u, \boldsymbol{\alpha}, \boldsymbol{\beta}, \boldsymbol{\gamma})$ such that $\succeq$ is represented by

$$
\begin{aligned}
V(A)=\max _{\boldsymbol{p} \in A}\left[\sum_{i \in I} \alpha_{i} u\left(p_{i}\right)\right. & +\beta_{1}\left(\max _{\boldsymbol{q} \in A} u\left(q_{1}\right)\right)\left(\alpha_{1}\left(u\left(p_{1}\right)-\max _{\boldsymbol{q} \in A} u\left(q_{1}\right)\right)\right) \\
& \left.-\beta_{S}\left(\max _{\boldsymbol{q} \in A} \sum_{i \in S} \alpha_{i} u\left(q_{i}\right)\right)\left(\sum_{i \in S} \alpha_{i} u\left(p_{i}\right)-\max _{\boldsymbol{q} \in A} \sum_{i \in S} \alpha_{i} u\left(q_{i}\right)\right)\right],
\end{aligned}
$$

\section{Examples}

\section{D.1 Example 2}

Fix a player $i \in I=\{1,2\}$. Let $j$ be the opponent of the player $i$. The following table (Table 4 ) is the payoffs of player $i$. Let the reference point of the game $\mathcal{G}$ by $\overline{\boldsymbol{u}}_{\mathcal{G}}$.

Table 4: A Prisoner's Dilemma Game with Image-Conscious Preferences

\begin{tabular}{|c|c|}
\hline Player $i \backslash$ Player $j$ & Cooperation \\
\hline Cooperation & $\left(1+\beta_{i}\left(\overline{\boldsymbol{u}}_{\mathcal{G}}\right)\right)+\left(1-\beta_{j}\left(\overline{\boldsymbol{u}}_{\mathcal{G}}\right)\right)$ \\
\hline Defection & $\left(1+\beta_{i}\left(\overline{\boldsymbol{u}}_{\mathcal{G}}\right)\right)(1+g)+\left(1-\beta_{j}\left(\overline{\boldsymbol{u}}_{\mathcal{G}}\right)\right)(-l)$ \\
\hline
\end{tabular}

Suppose that the opponent $j$ takes $C$. Then,

$$
\begin{aligned}
\left(1+\beta_{i}\left(\overline{\boldsymbol{u}}_{\mathcal{G}}\right)\right)+\left(1-\beta_{j}\left(\overline{\boldsymbol{u}}_{\mathcal{G}}\right)\right) & \geq\left(1+\beta_{i}\left(\overline{\boldsymbol{u}}_{\mathcal{G}}\right)\right)(1+g)+\left(1-\beta_{j}\left(\overline{\boldsymbol{u}}_{\mathcal{G}}\right)\right)(-l) \\
\Leftrightarrow\left(1-\beta_{j}\left(\overline{\boldsymbol{u}}_{\mathcal{G}}\right)(1+l)\right. & \geq\left(1+\beta_{i}\left(\overline{\boldsymbol{u}}_{\mathcal{G}}\right)\right) g \\
\Leftrightarrow\left(1-\beta_{j}\left(\overline{\boldsymbol{u}}_{\mathcal{G}}\right)\right. & \geq\left(1+\beta_{i}\left(\overline{\boldsymbol{u}}_{\mathcal{G}}\right)\right) \cdot \frac{g}{1+l}
\end{aligned}
$$

Hence,

$$
\beta_{j}\left(\overline{\boldsymbol{u}}_{\mathcal{G}}\right) \leq 1-\left(1+\beta_{i}\left(\overline{\boldsymbol{u}}_{\mathcal{G}}\right)\right) \frac{g}{1+l}
$$




\section{D.2 Example 3}

Fix a player $i \in I=\{1,2\}$. Let $j$ be the opponent of the player $i$. The following table (Table 5 ) is the payoffs of player $i$. Let the reference point of the game $\mathcal{G}$ by $\overline{\boldsymbol{u}}_{\mathcal{G}}$.

Table 5: A Prisoner's Dilemma Game with Image-Conscious Preferences

\begin{tabular}{|c|c|}
\hline Player $i \backslash$ Player $j$ & Defection \\
\hline Cooperation & $\left(1+\beta_{i}\left(\overline{\boldsymbol{u}}_{\mathcal{G}}\right)\right)(-l)+\left(1-\beta_{j}\left(\overline{\boldsymbol{u}}_{\mathcal{G}}\right)\right)(1+g)$ \\
\hline Defection & 0 \\
\hline
\end{tabular}

Suppose that the opponent $j$ takes $D$. Let the reference point of the game $\mathcal{G}$ by $\overline{\boldsymbol{u}}_{\mathcal{G}}$. Then,

$$
\begin{aligned}
\left(1+\beta_{i}\left(\overline{\boldsymbol{u}}_{\mathcal{G}}\right)\right)(-l)+\left(1-\beta_{j}\left(\overline{\boldsymbol{u}}_{\mathcal{G}}\right)\right)(1+g) & \leq 0 \\
\Leftrightarrow\left(1-\beta_{j}\left(\overline{\boldsymbol{u}}_{\mathcal{G}}\right)\right)(1+g) & \leq\left(1+\beta_{i}\left(\overline{\boldsymbol{u}}_{\mathcal{G}}\right)\right) \cdot l \\
\Leftrightarrow\left(1-\beta_{j}\left(\overline{\boldsymbol{u}}_{\mathcal{G}}\right)\right) & \leq\left(1+\beta_{i}\left(\overline{\boldsymbol{u}}_{\mathcal{G}}\right)\right) \frac{l}{1+g} .
\end{aligned}
$$

Hence, $\beta_{j}\left(\overline{\boldsymbol{u}}_{\mathcal{G}}\right) \geq 1-\left(1+\beta_{i}\left(\overline{\boldsymbol{u}}_{\mathcal{G}}\right)\right) \frac{l}{1+g}$ leads to take $D$. 


\section{References}

Andreoni, J. (1989): "Giving with Impure Altruism: Applications to Charity and Ricardian Equivalence," Journal of Political Economy, 1447-1458.

Andreoni, J. (1990): "Impure Altruism and Donations to Public Goods: A Theory of Warm-Glow Giving," Economic Journal, 100(401), 464-477.

Anscombe, F. J., And Aumann, R. J. (1963): "A Definition of Subjective Probability," Annals of Mathematical Statistics, 34(1), 199-205.

Baillon, A., Bleichrodt, H., And Spinu, V. (2018): "Searching for the Reference Point," Working Paper.

Bénabou, R., And Tirole, J. (2006): "Incentives and Prosocial Behavior," American Economic Review, 96(5), 1652-1678.

Bursztyn, L., And Jensen, R. (2017): "Social Image and Economic Behavior in the Field: Identifying, Understanding, and Shaping Social Pressure," Annual Review of Economics, 9, 131-153.

Bushong, B., Rabin, M., And Schwartzstein, J. (2017): "A Model of Relative Thinking," Unpublished Manuscript, Harvard University, Cambridge, MA.

Dana, J., Cain, D. M., And Dawes, R. M. (2006): "What you don't know won't hurt me: Costly (but quiet) exit in dictator games," Organizational Behavior and Human Decision Processes, 100(2), 193-201.

Dekel, E. (1986): "An Axiomatic Characterization of Preferences under Uncertainty: Weakening the Independence Axiom," Journal of Economic theory, 40(2), 304-318.

Dekel, E., Lipman, B. L., And Rustichini, A. (2001): "Representing Preferences with A Unique Subjective State Space," Econometrica, 69(4), 891-934.

Dillenberger, D., And Sadowski, P. (2012): "Ashamed to be Selfish," Theoretical Economics, 7(1), 99-124.

Doron, R., And Steverson, K. (2018): "Bad Temptation," Working Paper, available at SSRN: https://ssrn. com/abstract=3127575.

Evren, Ö., And Minardi, S. (2017): "Warm-Glow Giving and Freedom to be Selfish," Economic Journal, 127, Issue 603, 1381-1409.

Fehr, E., And Schmidt, K. M. (1999): "A Theory of Fairness, Competition, and Cooperation," Quarterly Journal of Economics, 817-868. 
GilboA, I. (2009): Rational Choice, MIT Press.

Gul, F., And Pesendorfer, W. (2001): "Temptation and Self-Control," Econometrica, 69(6), 1403-1435.

Hashidate, Y. (2019): “Axiomatization of Interdependent Preferences," Working Paper, Waseda University.

Hershey, J. C., And Schoemaker, P. J. (1985): "Probability versus Certainty Equivalence Methods in Utility Measurement: Are They Equivalent?," Management Science, $31(10), 1213-1231$.

Hiebert, P. G. (1985): Anthropological Insights for Missionaries, Baker Academic.

Kameda, T., Inukai, K., Higuchi, S., Ogawa, A., Kim, H., Matsuda, T., and SakAgAmi, M. (2016): "Rawlsian Maximin Rule operates as a Common Cognitive Anchor in Distributive Justice and Risky Decisions," Proceedings of the National Academy of Sciences, 113(42), 11817-11822.

Kawamura, T., Ogawa, K., and Osaki, Y. (2019): "Controlling Social Image through Dictating the Risk: Experimental Evidence," Working Paper.

Kopylov, I. (2009): "Finite Additive Utility Representations for Preferences over Menus," Journal of Economic Theory, 144(1), 354-374.

Köszegi, B., And Rabin, M. (2006): "A Model of Reference-Dependent Preferences," Quarterly Journal of Economics, 1133-1165.

Köszegi, B., And Szeidl, A. (2012): “A Model of Focusing in Economic Choice," Quarterly Journal of Economics, 128(1), 53-104.

Kreps, D. M. (1979): "A Representation Theorem for "Preference for Flexibility"," Econometrica: Journal of the Econometric Society, 565-577.

Kreps, D. (1988): Notes on the Theory of Choice, Westview Press.

LeA, S. E., And Webley, P. (1997): "Pride in Economic Psychology," Journal of Economic Psychology, 18(2-3), 323-340.

Minm, M., And OzBeK, K. (2018): "Mood-Driven Choices and Self-Regulation," Journal of Economic Economy, 176, 727-760.

Neilson, W. S. (2009): "A Theory of Kindness, Reluctance, and Shame for Social Preferences," Games and Economic Behavior, 66(1), 394-403. 
Noor, J., And Ren, L. (2015): “Temptation and Guilt,” Working Paper.

Noor, J., And Takeoka, N. (2015): "Menu-Dependent Self-Control," Journal of Mathematical Economics, 61, 1-20.

Ok, E. A., Ortoleva, P., And Riella, G. (2015): "Revealed (P) Reference Theory," American Economic Review, 105(1), 299-321.

Olszewski, W. (2007): "Preferences over Sets of Lotteries," Review of Economic Studies, $74(2), 567-595$.

Rabin, M. (1993): "Incorporating Fairness into Game Theory and Economics," American Economic Review, 1281-1302.

Rohde, K. I. (2010): “A Preference Foundation for Fehr and Schmidt's Model of Inequity Aversion," Social Choice and Welfare, 34(4), 537-547.

SAito, K. (2013): "Social Preferences under Risk: Equality of Opportunity versus Equality of Outcome," American Economic Review, 103(7), 3084-3101.

SAito, K. (2015): "Impure Altruism and Impure Selfishness," Journal of Economic Theory, 158, 336-370.

Segal, U., And Sobel, J. (2007): "Tit for Tat: Foundations of Preferences for Reciprocity in Strategic Settings," Journal of Economic Theory, 136(1), 197-216.

Sobel, J. (2005): "Interdependent Preferences and Reciprocity," Journal of Economic Literature, 43(2), 392-436.

Scheff, T. J. (1988): "Shame and Conformity: The Deference-Emotion System," American Sociological Review, 395-406.

SchmeIDleR, D. (1989): "Subjective Probability and Expected Utility without Additivity," Econometrica: Journal of the Econometric Society, 571-587.

Tadelis, S. (2011): "The Power of Shame and the Rationality of Trust," Working Paper.

Yagasaki, M. (2018): "Pride, Shame and Social Comparisons," Working Paper, available at SSRN: https://papers.ssrn. com/abstract=3414146. 